Florida International University FIU Digital Commons

$11-12-2014$

\title{
Hearing the Child's Voice: Their Lived Experience in the Pediatric Intensive Care Unit
}

Andrea S. Prentiss

Florida International University, rapare@aol.com

DOI: $10.25148 /$ etd.FI14110737

Follow this and additional works at: https:// digitalcommons.fiu.edu/etd

Part of the Critical Care Nursing Commons, and the Pediatric Nursing Commons

\section{Recommended Citation}

Prentiss, Andrea S., "Hearing the Child's Voice: Their Lived Experience in the Pediatric Intensive Care Unit" (2014). FIU Electronic Theses and Dissertations. 1633.

https://digitalcommons.fiu.edu/etd/1633

This work is brought to you for free and open access by the University Graduate School at FIU Digital Commons. It has been accepted for inclusion in FIU Electronic Theses and Dissertations by an authorized administrator of FIU Digital Commons. For more information, please contact dcc@fiu.edu. 


\title{
FLORIDA INTERNATIONAL UNIVERSITY \\ Miami, Florida
}

HEARING THE CHILD'S VOICE:

THEIR LIVED EXPERIENCE IN THE PEDIATRIC INTENSIVE CARE UNIT

\author{
A dissertation submitted in partial fulfillment of \\ the requirements for the degree of \\ DOCTOR OF PHILOSOPHY \\ in \\ NURSING \\ by
}

Andrea S. Prentiss

2014 
To: Dean Ora Lea Strickland

Nicole Wertheim College of Nursing \& Health Sciences

This dissertation, written by Andrea S. Prentiss entitled Hearing the Child's Voice: Their Lived Experience in the Pediatric Intensive Care Unit, having been approved in respect to style and intellectual content, is referred to you for judgment.

We have read this dissertation and recommend that it be approved.

Dionne Stephens

Jean Hannan

Dorothy Brooten

JoAnne Youngblut, Major Professor

Date of Defense: November 12, 2014

The dissertation of Andrea S. Prentiss is approved.

Dean Ora Lea Strickland Nicole Wertheim College of Nursing \& Health Sciences

Dean Lakshmi N. Reddi

University Graduate School

Florida International University, 2014 
C Copyright 2014 by Andrea S. Prentiss

All rights reserved. 


\section{DEDICATION}

This dissertation is dedicated to those hospitalized children who were willing to share their stories with me. This work was possible because of them. To Ryan, Evan, Anna, and Valerie - thank you for listening, encouraging, and understanding when work came before play. I would also like to dedicate this work to my good friend and colleague, Eve Butler. Thank you for paving the way and providing such encouragement and support.

To my mom, thank you for driving me home, listening to my stories, and centering me. You have always been my biggest cheerleader and fan. Thank you for believing in me, sometimes more than I believed in myself. We do not need to scratch the 2014 date off the mugs! I love you so much.

To my best friend and husband, Richard; words are not enough to express my gratitude to you. You were patient, encouraging, tolerated my moods, helped with housework, and provided valuable table expertise. If not for you, I would have given up long ago. Now you have me back. I love you!

And lastly, to my new little granddaughter Raelyn who was born during this very challenging time. You have provided a much deeper meaning and purpose to my life. You gave me a goal, finish! Although your mommy was wonderful with daily pictures texting and video chat is not the same as cuddling.

Thank you all so much! 


\section{ACKNOWLEDGMENTS}

I would like to acknowledge and thank the hospitalized children who shared their innermost thoughts with me. This dissertation would not have been possible without their willingness to participate. I have been blessed with many friends and colleagues who have helped me through this journey. I would like to thank my research personnel (Jamie, Carrie, Mariana, and Carolina), unit manager (Louise), and PICU nurses for their assistance and desire to ensure I was aware of potential participants - your phone calls were so welcomed. Donna, my data abstractor, I would not have been able to do this without her attention to detail and timeliness! She made it possible to concentrate on other work. To all my wonderful friends who stopped me and asked how I was doing, thank you for listening and encouraging me. Many people provided necessary behind the scenes assistance: my translators (Rosalia and Tony), information technology friend (Wilhelm) who provided valuable knowledge for trouble shooting frustrating computer glitches, and my director, Michelle, who understands the value of research.

I would especially like to recognize my committee chair, Dr. Joanne Youngblut. Her expertise and guidance have provided me with a much richer research understanding and writing ability. It has been such an honor and privilege to work with someone so esteemed in pediatric research. I look forward to many more years of her mentorship.

I would like to thank my committee members Dr. Dorothy Brooten, Dr. Jean Hannan, and Dr. Dionne Stephens for their expertise, time, and kind words during this experience. I am especially grateful to my dear friend Rosa Roche. Her support and mentorship provided a much needed light when it was difficult to see. Finally, I would like to thank my friends and family for their love and support. 


\begin{abstract}
OF THE DISSERTATION
HEARING THE CHILD'S VOICE:

THEIR LIVED EXPERIENCE IN THE PEDIATRIC INTENSIVE CARE UNIT
\end{abstract}

by

\author{
Andrea S. Prentiss \\ Florida International University, 2014 \\ Miami, Florida \\ Professor JoAnne Youngblut, Major Professor
}

Background: More than 200,000 children are admitted annually to Pediatric Intensive Care Units (PICUs) in the US. Research has shown young children can provide insight into their hospitalization experiences; child reports rather than parental reports are critical to understanding the child's experience. Information relating to children's perceptions while still in the PICU is scarce.

Aims: The purpose of this qualitative study was to investigate school age children's and adolescents' perceptions of PICU while in the PICU; changes in perceptions after transfer to the General Care Unit (GCU); differences in perceptions of school age children/adolescents and those with more invasive procedures.

Methods: Interviews were conducted in PICU within 24-48 hours of admission and 24-48 hours after transfer to GCU. Data on demographics, clinical care and number/types of procedures were obtained.

Results: Participants were 7 school age children, 13 adolescents; 10 Hispanic; 13 males. Five overarching themes: Coping Strategies, Environmental Factors, Stressors, Procedures/Medications, and Information. Children emphasized the importance of peer 
support and visitation; adolescents relied strongly on social media and texting. Parent visits sometimes were more stressful than peer visits. Video games, TV, visitors, and eating were diversional activities. In the PICU, they wanted windows to see outside and interesting things to see on the ceiling above them. Children expressed anticipatory fear of shots and procedures, frustration with lab work, and overwhelming PICU equipment. Number of child responses was higher in PICU (927) than GCU (593); the largest difference was in Environmental Factors. Variations between school age children and adolescents were primarily in Coping Strategies, especially in social support. Number of GCU procedures were the same ( 8 children) or greater ( 2 children) than PICU procedures.

Discussion: Admission to PICU is a very stressful event. Perceptions from children while still in PICU found information not previously found in the literature. Longitudinal studies to identify children's perceptions regarding PICU hospitalization and postdischarge outcomes are needed. 


\section{TABLE OF CONTENTS}

CHAPTER

I.

INTRODUCTION .............................................. 1

Purpose ..................................................... 1

Significance................................................. 2

PICU Environment, Stressors, and Memories....................... 7

Responses to PICU Hospitalization............................... 10

Delirium............................................. 12

Post-Traumatic Stress.................................... 12

Conceptual Framework....................................... 14

Research Questions.......................................... 19

II. REVIEW OF THE LITERATURE............................. 20

Children's Understanding of Illness............................ 21

Healthy Children........................................ 24

Ill Children.............................................. 33

PICU Environment: Stressors, Fears, and Memories............... 40

Stressors.............................................. 40

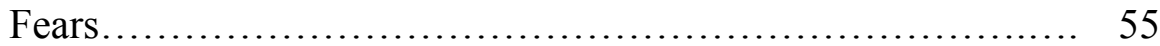

Memories.............................................. 63

Child Responses to PICU................................... 71

Factual and Delusional Memory.............................. 77

Delirium and ICU Psychosis............................... 81

Post-Traumatic Stress..................................... 86

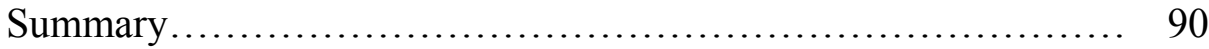

Issues and Gaps......................................... 92

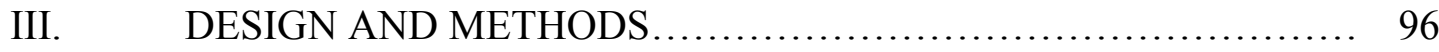

Settings........................................................ 96

Sample and Sampling Procedures............................. 97

Inclusion Criteria............................................ 98

Exclusion Criteria......................................... 98

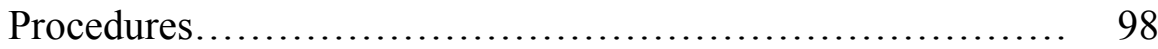

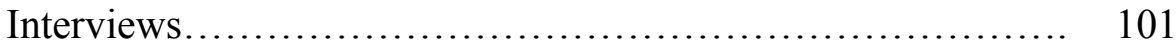

Data Collection Forms......................................... 104

Demographics........................................ 104

Invasive Procedure Score ................................. 105

Data Management and Analysis................................ 106

Parent Demographics and Chart Data........................ 106

Interviews............................................ 106

Research Questions........................................ 107

Human Subjects Protection................................... 108 
IV. $\quad$ RESULTS................................................... 110

Description of the Sample...................................... 110

Perceptions of PICU Hospitalization.............................. 116

Perceptions in PICU......................................... 116

Perceptions in GCU ...................................... 129

Perceptions Over Time.................................... 138

School Age Children and Adolescent's Perceptions............... 142

Invasive Procedure Scores...................................... 153

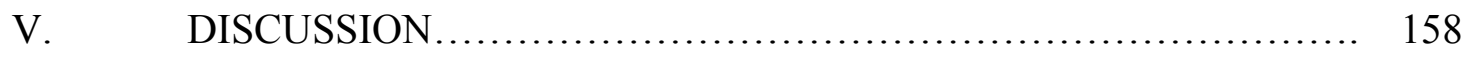

Discussion of Findings......................................... 159

Significance.................................................. 174

Limitations of the Study....................................... 176

Implications for Future Research................................ 177

Implications for Nursing Education and Practice.................. 179

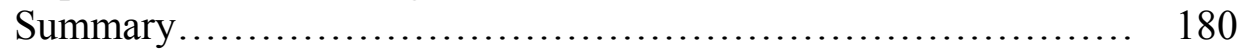

REFERENCES ............................................. 181

APPENDICES.................................................. 201

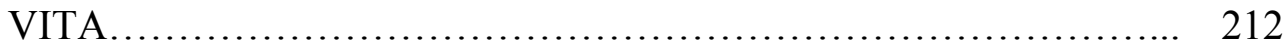




\section{LIST OF TABLES}

TABLE

PAGE

1. Description of PICU and GCU Participants......................... 112

2. Pediatric Intensive Care Descriptions................................ 113

3. PICU Interview Questions...................................... 115

4. Perceptions over Time (PICU to GCU).......................... 140

5. Coping Strategies by Age and Unit............................... 144

6. Environmental Factors by Age and Unit........................... 146

7. Stressors by Age and Unit..................................... 148

8. Procedures/Medications by Age and Unit......................... 150

9. Information by Age and Unit................................. 152

10. IPS Groups: PICU to GCU................................... 154

11. PICU IPS Groups and Themes................................. 156

12. GCU IPS Groups and Themes.................................. 157 


\section{CHAPTER I \\ INTRODUCTION}

Approximately three million children were hospitalized in the United States between 2007 and 2009 (Centers for Disease Control and Prevention, 2010) with more than 200,000 children in the United States admitted annually to a Pediatric Intensive Care Unit (PICU) for treatment of critical conditions (Odetola, Clark, Freed, Bratton, \& Davis, 2005; Rennick et al., 2011). Children in the PICU range from newborns through 21 years of age (American Academy of Pediatrics, 2012) and have a wide range of complex, progressive, and unstable medical, surgical, congenital, and traumatic conditions. These critically unstable, highly vulnerable infants, children, and adolescents rely on teams of highly skilled pediatric professionals to provide the latest in the art and science of critical

care for best outcomes. Advances in pediatric critical care have resulted in more children surviving critical illness but experiencing significant morbidity long after discharge affecting their quality of life and functional health (Jones et al., 2006; Muranjan, Birajdar, Shah, Sundaraman, \& Tullu, 2008; Namachivayam et al., 2010; Shudy et al., 2006; Taylor, Butt, \& Ciardulli, 2003).

\section{Purpose}

Illness and hospitalization may pose the first crisis a child encounters. The experience creates a dramatic change in the child's daily life and may affect their worldview and quality of life. Classic works of Bibace and Walsh (1980) found the impact of illness and subsequent hospitalization on child outcomes likely varies with the child's age, developmental level, disease acuity, existing support systems, and understanding of illness and death. Paramount to understanding the child's perception of 
illness is the need to understand their evolving cognitive development and conceptual change relating to their hospitalization experience. The purpose of this qualitative, phenomenological study was to investigate school-age children's and adolescents' perceptions of PICU hospitalization (a) in the PICU - within 24 - 48 hours of their being alert and able to speak and, (b) in the GCU - within $24-48$ hours following their transfer from the PICU.

\section{Significance}

Research investigating parent and health care provider perceptions of the hospitalized child has identified concerns, stressors, and psychological outcomes of the parents (Curley, 1988; Melnyk et al., 2004; Melnyk \& Feinstein, 2001; Small \& Melnyk, 2006). However, differences have been reported between the perceptions of the child and those of the parent (Cremeens, Eiser, \& Blades, 2006; Marino et al., 2009; Russell, Hudson, Long, \& Phipps, 2006; Sheffler, Hanley, Bagley, Molitor, \& James, 2009; Sundblad, Saartok, \& Engstrom, 2006; Vetter, Bridgewater, \& McGwin, 2012) and medical providers (Marino et al., 2009) with proxy-reports underestimating the ill child's quality of life when comparing child self-reports and parent proxy-reports. In a longitudinal study, Rajmil, López, López-Aguilà, and Alonso (2013) found moderate to low parent-child agreement at baseline and three year follow-up on health-related quality of life. Little research has focused on the children experiencing the pain and fear of hospitalization, often in isolation, as the family is coping with the frequently unanticipated admission and their child's critical condition. Research has demonstrated that very young children can provide insight into the hospitalization experiences (Irwin \& 
Johnson, 2005; Varni, Limbers, \& Burlwinkle, 2007a; Varni, Limbers, \& Burlwinkle, 2007b).

Parental needs when their child is hospitalized have been identified as provision of factual and timely information concerning their child's illness, equipment, and plan of care; being provided a sense of hope; being allowed to stay with their child at all times and participate in the child's care; and staff being sensitive to the parents' basic needs, rest and nutrition (Board \& Ryan-Wenger, 2000; 2003; Miles \& Carter, 1985). Stressors for parents include uncertainty over their child's outcome, receiving inconsistent information; alteration in their parental role, seeing the child in pain, not knowing how or being unable to communicate with and help the child, their child's appearance, and access to their child (Balluffi et al., 2004; Board \& Ryan-Wenger, 2000; Miles \& Carter, 1989; Rennick, 1986). Children have their own perspectives on their experiences which need to be investigated to identify their needs and stressors.

Children are forced to rapidly assimilate their experiences as they are exposed to medications, procedures, and equipment with little or no warning. They hear a barrage of sounds from various machines, equipment, monitors, healthcare providers, and other patients and families (Baily \& Timmons, 2005). Telephones ringing, doors opening and closing, chest tubes bubbling, EKG machines beeping, intravenous pumps alarming, suction sounds from nasogastric tubes, beepers, and voices as well as noises outside the child's immediate room or area further overwhelm their auditory senses (Fontaine, Briggs, \& Pope-Smith, 2001). Health care providers often speak using medical terminology not taking the time to ensure the child understands. Parents may appear extremely stressed and perhaps crying. 
Children may be pharmacologically paralyzed, while continuing to be aware of their surroundings, hearing and feeling everything, often without adequate sedation. Less than therapeutic levels of sedation essentially have the child trapped in a body without any voice or control over their experience. An endotracheal tube passing through their vocal cords is not only extremely uncomfortable but renders them unable to speak. They may be tied down in uncomfortable, unnatural positions. Younger children, taught not to talk to strangers, find themselves surrounded by and being cared for by strangers.

Children experience a lack of privacy and control, so vital to the adolescent, surrounded by strangers during their most vulnerable, personal times. They experience a loss of self-determination, needing permission for ordinary activities. Sleep, so vital to healing, is often disrupted by continuous loud noises and unnatural lighting (Carno \& Connolly, 2005; Coyne, 2006), their illness, medication administration, and care activities (Carno \& Connolly, 2005).

Children hospitalized in the PICU now find a loss of their normal social support. They are separated from their families, pets, and familiar surroundings (Coyne, 2006). Their friends and young siblings may not be allowed to visit, and the number of family members may be restricted. Many hospitals maintain restrictive visitation policies based on concerns about the patient's physiological stress, the ability to provide patient care, and fatigue for the family (Berwick \& Kotagal, 2004).

Long-term psychological outcomes of a PICU admission have been reported. Age and illness severity influence these outcomes, but the number of invasive procedures (Rennick \& Rashotte, 2009) is the strongest predictor of psychological distress for as long as 6 months following PICU admission (Rennick, Johnston, Dougherty, Platt, \& 
Ritchie, 2002). Pain also has long-term negative effects in children (Cohen et al., 2001; Johnson, Bournaki, Gagnon, Pepler, \& Bourgault, 2005; Klein, Gaspardo, Martinez, Grunau, \& Linhares, 2009). Children hospitalized in the PICU experience procedures such as insertion and removal of intravenous catheters (peripheral, arterial, central), urinary catheters, chest tubes, artificial airways, and intracranial monitors; suctioning; dressing changes; tape removal; spinal taps; rectal temperatures and probes, and multiple heel and/or finger sticks. Rennick et al. (2002) found children experienced as many as $160(M=89)$ invasive procedures a day.

Although health care personnel perceive more minor procedures such as taking temperatures and removing intravenous (IV) catheters as noninvasive, children perceive them quite differently (Rennick et al., 2002) and display anticipatory distress to needles and other invasive procedures (Blount et al., 2009; Coyne, 2006; Taddio, Shah, GilbertMacLeod, \& Katz, 2002). Pharmacological interventions are typically used for pain in the PICU; however, children still experience pain from invasive procedures in spite of analgesia. Painful invasive procedures greatly compromise physiological stability (Turner, 2005).

Research reports the child's appearance is a major stressor for parents with children in the PICU (Balluffi, Kassam-Adams, Kazak, Tucker, Dominguez, \& Helfaer, 2004; Board \& Ryan-Wenger, 2000; Miles \& Carter, 1989; Rennick, 1986; Youngblut \& Brooten, 2006; 2008; Youngblut, Brooten, \& Kuluz, 2005); however this may be a stressor for the child as well. Alteration in body image, so important to the adolescent, may be a result of trauma or surgery. Additionally many of the procedures the child experiences are not only a tangible reminder of the severity of their condition but also 
affect the child's appearance resulting in a very intimidating, frightful effect for the children and their families (Haines et al., 1995).

Children hospitalized in a PICU worry they may never get better; for many of these children the hospitalization is one of many. They worry something else may go wrong with them and/or they may have to be readmitted to PICU. Some children experience guilt that something they did or did not do caused their illness and subsequent PICU admissions. Children worry about their mortality, particularly those with chronic life-threatening illness (Rennick, McHarg, Dell’Api, Johnston, \& Stevens, 2008).

Few studies have examined the impact of the PICU environment, stressors, and memories of the overall experience on children from their perspective. Research relating to the child's hospitalization experience has typically been reported by parents or healthcare providers. However, proxy reports for both adults and children are not equivalent to self-report (Aastha, 2012; Alonso et al., 2010; Varni, Limbers, \& Burlwinkle, 2007). In the case of children, proxy reports are usually from the parents, most often the mothers, whose perceptions of child health and well-being are affected by their own concerns, distress, and related factors (Berg-Nielson, Vika, \& Dahl, 2003; De Los Reyes \& Kazdin, 2004). Based on an earlier body of work, pediatric patients' selfreport of pain intensity became the standard for clinical research and practice in the early 1990s following study results demonstrating that children as young as 5 years are able to accurately self-report pain levels using age-appropriate visual analog scale instruments (Varni \& Bernstein, 1991; Varni, Thompson, \& Harrison, 1987). Varni, Limbers, and Burlwinkle (2007) agree that parent proxy-report should be used when pediatric patients are too young, too cognitively impaired, too ill, or fatigued to complete a HRQOL 
instrument. However, parent reports cannot substitute for child self-report when the child is willing and able to provide their perspective. Research using child reports rather than parental reports are critical to understanding the child's experience.

Despite improved outcomes and survival rates, between $16 \%$ and $28 \%$ of children experience deterioration of emotional well-being following PICU hospitalization (Rennick et al., 2002; Rennick et al., 2004; Rennick et al., 2011; Rennick et al., 2008; Rennick \& Rashotte, 2009); however, children's appraisals and understanding of their PICU experiences are not well described. Research indicates that children hospitalized in the PICU have more adverse responses than those hospitalized in a General Care Unit (GCU). Despite significant advances in health care and improved survivor rates, gaps remain in the research pertaining to the impact of the PICU hospitalization on the child's psychological, emotional, and physical responses.

\section{PICU Environment, Stressors, and Memories}

Hospitalization is a stressful, individually unique event for children. An ICU admission is particularly unpredictable for critically ill children and their families as they experience dramatic swings in the child's condition (Shudy et al., 2006). Earlier reports of parental stress following emergent PICU admission found early parental anxiety elevated to near panic levels, eventually declining and stabilizing over days in the PICU (Huckabay \& Tilem-Kessler, 1999). Although parental levels of stress to PICU environmental stimuli decrease over time, increases in stressors related to staff communication and behavior are seen (Long, 2003; Huckabay \& Tilem-Kessler, 1999).

Parents of intubated children were found to experience greater stress from painful procedures (Haines et al., 1995), changes in parental role, and staff communication 
(Smith, Hefley, \& Anand, 2007) than parents of non-intubated children. Parents of nonintubated children experienced higher stress from their children's emotional and behavioral responses (Haines et al., 1995). High levels of maternal stress were found to have a negative impact on children's coping skills (Curley, 1988; Curley \& Wallace, 1992; Melnyk et al., 2004) and inhibit effective parenting (Small \& Melnyk, 2006) leading to high anxiety levels in the child (Melnyk \& Feinstein, 2001).

The majority of PICU admissions are emergent and unplanned. The timing and severity of the child's illness, as well as the vast number of procedures and tests done in a very brief time, are key factors in the resultant parental stress levels (Huckabay \& TilemKessler, 1999). In developing the Parental Stressor Scale: Pediatric Intensive Care Unit (PSS: PICU), Miles and Carter (1982) classified sources of stress experienced by parents with children in PICU as situational, personal characteristics, and environmental stimuli. Studies using the PSS: PICU scale found the most severe parental stress from the procedures their child endures and alterations in their parent role as they experience a sense of helplessness in advocating for and protecting their children (Board, 2004; Board \& Ryan-Wenger, 2003; Long, 2003). Other identified stressors include alterations in the child's appearance, alarms, nursing procedures, and communication difficulties with PICU staff (Balluffi et al., 2004; Miles \& Carter, 1982). Comparing PICU parents to GCU parents, Youngblut and colleagues found that mothers in the PICU group reported more stress than the GCU group on three of the seven PSS: PICU subscales than the GCU group: child's appearance, sights and sounds of the unit, and procedures done to the child (Youngblut \& Brooten, 2008; Youngblut, Brooten, \& Kuluz, 2005). Research by Board and colleagues (Board, 2004; Board \& Ryan-Wenger, 2003) also found parents of 
children in the PICU reported higher levels of stress for procedures and sights and sounds of the unit than parents of children in the GCU. Parental stress symptoms and coping issues remain evident long after their child's critical illness (Board, 2004; Board \& RyanWenger, 2002; Youngblut \& Brooten, 2008).

Stressors experienced by children include procedures, tubes, pain, lack of sleep, machinery, other sick children, environmental factors, loss of control, and threat to life and physical integrity. Optimal healing is impeded by multiple barriers in the PICU, especially very high noise levels (Carvalho, Pedreira, \& deAguiar, 2005; Milette \& Carnevale, 2003). The constant assault of noise in the ICU environment on patients vulnerable from the physical insult of surgery or disease is associated with physiological manifestations, such as sleep disruption, which may persist following discharge (AlSamsam \& Cullen, 2005; Carno, Hoffman, Henker, Carcillo, \& Sanders, 2004; Carno \& Connoly, 2005). The continuous bright lighting disrupts circadian rhythm which may decrease melatonin levels impeding children's recovery (Fontaine et al., 2001).

The trajectory and treatment of critical conditions expose children to a wide range of extreme stressors which include painful, frightening invasive procedures; distressing environmental events; threat to life and physical integrity; ventilators; intravenous pumps; bright lights; strangers; other sick children (Dow, Kenardy, Long, \& LeBrocque, 2012); respiratory insufficiency; separation from families; loss of control; and delirium with potential for psychotic episodes (Davydow, Richardson, Zatzick, \& Katon, 2010). Younger children may view large, noisy machines as extensions of themselves believing all PICU equipment controls their bodily functions (Vanek, 1979). High procedure rates are an additional stressor for children in the PICU with the majority of the research 
finding a positive correlation between invasive procedures and adverse outcomes, such as delirium, post-traumatic stress (PTS), or post-traumatic stress disorder [PTSD] (Rennick \& Johnston, 2000; Rennick et al. 2011; Rennick et al., 2002; Rennick et al., 2004).

Early research found children may have highly distorted memories of their PICU experiences (Barnes, 1975). Multiple factors are implicated in alteration of their memories which include cognitive level, resource availability, medication use, and sleep deprivation. The majority of the research studies have found that children are able to recall many aspects of their PICU stay, find it highly anxiety-provoking (Board, 2005; Karande, Kelkar, \& Kulkarni, 2005; Muranjan et al., 2008; Playfor, Thomas, \& Choonara, 2000), and experience intrusive thoughts such as bad dreams or trouble with sleeping from recurrent images or thoughts of their PICU hospitalization (Muranjan et al., 2008). The PICU environment and requisite care increases the probability that children will experience delusional memories placing them at risk for negative outcomes (Colville, 2004, 2008; Colville \& Gracey, 2005; Colville, Kerry, \& Pierce, 2008; Colville \& Pierce, 2005; Colville, Tighe, \& Pierce, 2006).

\section{Responses to PICU Hospitalization}

The hospital environment, particularly the PICU, places a child at high risk for physical, psychological, and social stress and may lead to emotional and behavioral coping responses, especially internalizing behaviors (e.g. anxiety, depression, and somatization) and externalizing behaviors (e.g. hyperactivity and aggression; Small \& Melnyk, 2006). These responses may be manifested upon admission or post discharge as changes in temperament, delirium, coping, PTS, or PTSD. Research has indicated these adverse psychological responses continue long after the child's hospital discharge 
(Melnyk, 1994; Melnyk et al., 2004; Playfor et al., 2000; Rennick et al., 2002). Findings on the psychologic impact of children's PICU hospitalization depict both resilience and distress (Colville, 2008), and children may manifest long-term emotional dysfunction, negative physical health outcomes, and poorer health-related quality of life (Dow et al., 2012).

Children's physical, emotional, social, and cognitive development helps to shape their perception of the stressful hospital experience (Aley, 2002). Factors negatively affecting a child's coping response to hospitalization include being younger and female, and family factors such as highly anxious and less educated mothers, increased numbers of family stressors, and lower socioeconomic status (Small \& Melnyk, 2006). Melnyk (2000) found that children's emotional and psychological predisposition, coping skills, and prior hospital experiences affect the child's responses. Health care experiences like hospitalization, can result in developmental delays, loss of newly acquired developmental skills, loneliness, isolation, and PTS for the child (Melnyk, 1994; Aley, 2002).

Developmentally appropriate fears, such as stranger anxiety and fear of the dark in preschool children and lack of privacy and decreased autonomy in the adolescent, also greatly affect children's negative responses to hospitalization. Children of all ages reported fear of pain (needles) and procedures (Carney et al., 2003; Koening, Chesla, \& Kennedy, 2003; Lindke, Nakai, \& Johnson, 2006; Salmela, Salanterä, \& Aronen, 2009; Wennström \& Bergh, 2008).

Coping responses reported prior to and after hospitalization include developmental regression, withdrawal, sleep disturbances, and hyperactivity or aggression. Children with acute illnesses experienced more behavioral problems while 
hospitalized. Those with chronic conditions had greater behavior problems out of the hospital. The latter may be explained by greater familiarity with the hospital environment and development of coping skills (Melnyk \& Feinstein, 2001; Small \& Melnyk, 2006). Regardless of hospitalization experience, almost all children reported feelings of uncertainty and needing protection (Wilson, Megel, Enenbach, \& Carlson, 2010).

\section{Delirium}

Delirium or ICU psychosis, widely studied in adult ICU patients, has received little attention in critically ill children. Research indicates children manifest a variety of delirium symptoms, including sleep disturbances; confusion; and impaired attention, concentration, and responsiveness. Additional symptoms included irritability, affective lability, impaired orientation, agitation, apathy, anxiety, impaired memory, and hallucinations, closely mirroring symptoms in older patients. Delirium has been linked to medications, surgery and anesthesia, infections, cancer, and trauma (Colville et al., 2008; Grover et al., 2012; Smeets et al., 2010; Turkel \& Tavares, 2003).

Children with delirium were found to be older with longer ventilator times (Smeets et al., 2010). They generally have longer stays; however, it is not clear whether longer stays lead to children's developing delirium, or whether delirium results in longer hospital stays (Colville et al., 2008; Grover, 2012; Smeets et al., 2010; Turkel \& Tavare', 2003).

\section{Post-Traumatic Stress}

PTSD has been increasingly used as a diagnostic framework for evaluating children's psychological responses to PICU hospitalization. Research, although minimal, 
indicates children experience symptoms of PTS resulting in high levels of distress and psychological impairment. Symptoms may persist over time resulting in long-term emotional dysfunction, adverse physical health outcomes, and poorer health-related quality of life (Landolt, Buehlmann, Maag, \& Schiestl, 2009; Seng, Graham-Berman, Clark, McCarthy, \& Ronis, 2005). Children in PICU are difficult to assess for PTS due to the nature of the environment and their conditions. PICU staff are vigilant for physiologic changes and may interpret agitation, crying, confusion, anger, and aggression as well as totally opposite behaviors (detachment and withdrawal) as secondary symptoms of a physiologic event such as pain rather than PTS (Dow et al., 2012).

Early PTS symptoms include nightmares, intense emotional reactions, elevated heart rate and blood pressure, exaggerated startle reflex, irritability, anger, restlessness, and sleep disturbances. Children also may appear frozen, detached, or unresponsive and may exhibit decreased awareness of their surroundings with early trauma responses (Scheeringa, Zeanah, \& Cohen, 2011). Research indicates many children continue to experience significant PTS as long as 9 months past discharge (Bronner, Knoester, Bos, Last, \& Grootenhuis, 2008; Rennick et al., 2002).

Development of PTSD in children admitted to the PICU is related to increased parental distress, greater length of PICU stay, delusional memories, and emergency admission (Colville \& Pierce, 2005; Colville et al., 2006; Connolly, McClowry, Hayman, Mahony, \& Artman, 2004; Rennick et al., 2002, 2004; Small \& Melnyk, 2006). Compared to those in the GCU, PICU survivors had higher risk for increased medical fears, decreased control over their health, and more PTSD symptoms, especially intrusive thoughts with nightmares, insomnia, and emotional reactions (Rees, Gledhill, Garralda, \& 
Nadel, 2004; Rennick et al., 2004). Factors relating to PTSD symptoms included being younger than 7 years, more severely ill, and experiencing greater numbers of invasive procedures (Rennick \& Johnston, 2000). Children's PTSD often goes unrecognized as symptoms may be attributed to cognitive and emotional psychological development.

\section{Conceptual Framework}

This exploratory, qualitative study was guided by the Transactional Model of Child Development first described by Sameroff and Chandler (1975). The underlying assumptions of this model are that contact between the person and environment is a transaction in which each is altered by the other. This model explains the development of positive and negative outcomes for children through the interaction of nature and nurture. The child's development is a product of the continuous, dynamic interactions of the child and their experiences through their social settings. Core to the transactional model is the bidirectional, interdependent effects of the children and their environments (Sameroff, 2009, p.5). Research is needed on the relationship between child characteristics and experience characteristics and their effects on children's PICU hospitalization perceptions.

Sameroff and Chandler (1975) described three models for understanding developmental consequences of early childhood trauma; main effects, interactional, and a transactional model. Constitution, or environment, is considered the sole contributors to later developmental outcomes in the main effects model; however, linear chains of causality were not found in research addressing the main effect models. Children with delivery complications do not become retarded and children of schizophrenics do not become schizophrenic. These findings led researchers to reject the main effects model 
and adopt an interactional model in which constitution and environment are considered together. Children with good constitutions raised in good environments will have the best outcomes and children with poor constitutions raised in poor environments will have the worse outcomes. Intermediate outcomes will be seen in children with good constitutions raised in poor environments and vice versa. However, many children growing up in atrocious environments achieved great outcomes while, conversely children with no identifiable constitutional problems raised in the optimum environments develop serious deviancies later in life. The main effects and interactional models assume the aspects of nature and nurture is constant over time. The transactional model was developed to explain the variety of effects found between and within constitutional and environmental variables using a dynamic approach; it deals with the processes as well as the outcomes of development (pp. 66-67). Child outcomes were then able to be explained with the interactional model; good parenting could compensate for infant problems, and well-behaved children could offset the parental propensity for abuse. Sameroff (2009) described children as being in a constant state of active reorganization who do not necessarily maintain habits or inborn traits as a static characteristic. The child's interaction with their environment helps to define their developmental changes (p.8).

Magee and Roy (2008) examined early childhood risk factors on school-age behavior used the Transactional Model of Child Development (Sameroff \& Chandler, 1975 ) in 521mother-child-environment triads. The sample was a secondary analysis from the 8-year National Longitudinal Survey of Youth. The sample was a middle class, low risk population. They found temperament, gender, and parenting ability in early 
childhood as significant predictors of later behavior problems in children. A young toddler with all three predictors of risk - male gender, early difficult temperament, and a less able parent - was eight times more likely to have behavior problems at school.

Callender, Olson, Choe, and Sameroff (2012) examined risk for child externalizing behavioral problems in 245 children using parent and teacher report in a longitudinal study. Children were approximately 3 years old at Time 1 (T1) and 5 1/2 years old at Time 2 (T2). Mothers and fathers reported their depressive symptoms, perceptions of their child's reciprocal affection and responsiveness, frequency of physical punishment, and child externalizing problems at T1. The child's externalizing behaviors were rated by parents and teachers at T2. Parents' negative attributions mediated positive relations between their depressive symptoms and frequency of physical punishment for both parents. Results indicated more frequent parental physical punishment during early preschool years predicted higher levels of child externalizing problems in kindergarten. Individual child characteristics, such as gender, developmental level, and temperament, play significant roles in behavior problems (Caspi et al., 2003). Additionally, parent characteristics and parenting styles influence child behaviors (Barry, Dunlap, Cotton, Lochman, \& Wells, 2005) with physically aggressive parenting and low levels of warmth linked to child aggressive and oppositional behaviors (Stormshak, Bierman, McMahon, \& Lengua, 2000). Children's coping skills are negatively affected when mothers have lower education and socioeconomic levels (Logsdon, Birkimer, Ratterman, Cahill, \& Cahill, 2002; Small \& Melnyk, 2006) and increased levels of stress (Curley, 1988; Curley \& Wallace, 1992; Melnyk et al., 2004). Heightened anxiety inhibits mothers from parenting effectively during hospitalization; maternal state anxiety 
is the most significant predictor of children's aggressive and hyperactive behavior following PICU hospitalization (Small \& Melnyk, 2006) with high maternal anxiety leading to high anxiety levels in the child (Melnyk \& Feinstein, 2001). Interestingly, parents with previous PICU experience reported higher levels of stress on both parental role and total scores (Curley \& Wallace, 1992) consistent with Youngblut and Jay's (1991) study findings that parents with previous PICU experience worried more with repeat PICU admission.

Research indicates children who are younger, more severely ill, and exposed to more invasive procedures are at higher risk for increased medical fears, a lower sense of control over their health, and more symptoms of PTS six months following hospital discharge (Rennick et al., 2002). Associations have been found between higher anxiety levels and longer intubation time (Board, 2005); between pediatric delirium and use of sedatives/analgesics (Schieveld et al., 2007); between PTS and illness severity and number of invasive procedures (Rennick et al., 2004); between PTSD and longer duration of opiate/benzodiazepine use (Colville \& Pierce, 2005); and between longer lengths of PICU stay and PTSD (Connolly et al., 2004). Child and clinician-administered reports found children hospitalized in PICU with higher rates of PTS symptoms than children hospitalized in GCUs (Rees et al., 2004) and delusional experiences, and higher rates of PTS were seen with longer periods of sedation (Colville et al., 2008). Research also indicates deterioration in children's quality of life following PICU discharge (Jayashree, Singhi, \& Malhi, 2003; Jones et al., 2006; Taylor et al., 2003).

To predict child outcomes, the child's individual characteristics as well as the characteristics of the parent must be considered within the context of their environment. 
The conceptual model for this study reflects the transactional model (see Figure 1) and consists of three constructs - the parents, child, and environment - which continuously interact in a bidirectional manner during the PICU hospitalization. The transactional model identifies children's development as occurring within the context of the environment and, to explain outcomes, the child's individual characteristics as well as characteristics of the environment need to be considered (Magee \& Roy, 2008). Child outcomes following PICU hospitalization cannot be explained by the underlying characteristics of the child or the parents alone but must include both real and perceived critical care experiences.

\section{Figure 1}

\section{Transactional Model of Child Outcomes Following PICU Hospitalization. Sameroff and Chandler, 1975}

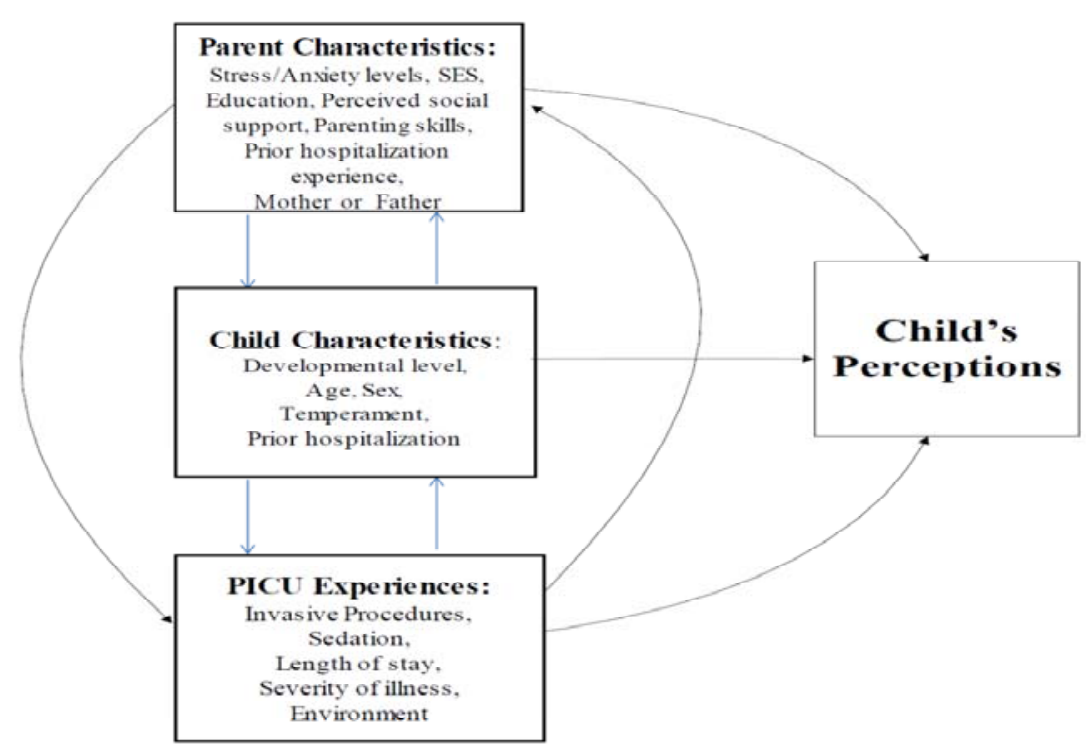




\section{Research Questions}

This study was designed to answer the following research questions:

1. What are children's perceptions of their PICU hospital experiences while in the PICU and the GCU?

2. How do children's perceptions of their PICU experiences change over time; PICU to GCU?

3. Do these perceptions vary for school age children and adolescents, and for those with a higher number of invasive procedures?

Repeated interviews allowed for perceptions of the same sample of participants to be examined following PICU admission and following their transfer from the PICU to the GCU. Content analysis was used to identify themes in children's and adolescents' descriptions of their PICU experiences and how their perceptions varied over time and with age and number of invasive procedures. 


\section{CHAPTER II}

\section{REVIEW OF THE LITERATURE}

Admission to the Pediatric Intensive Care Unit (PICU) produces anxiety and stress for children and their families with the sterile, dehumanizing environment exacerbating negative experiences. Care for the child occurs with intense monitoring in close proximity to nurses and other patients with environmental effects of noise, lights, and frequent interruptions common. Impeccable critical care skills, advanced knowledge, and state of the art technology are not the only factors influencing the child's recovery.

Children and their families experience negative psychological and physiological responses as a result of pain, injury, serious illness, medical procedures, and invasive or frightening treatments incurred when in the PICU. Multiple psychosocial factors affect the child's response to PICU hospitalization. These include the child's physical, emotional, social, cognitive development, age, and sex (Aley, 2002; Rennick et al., 2004). Family factors influencing children's outcomes included lower socioeconomic status, increased number of family stressors, and highly anxious and/or less educated mothers (Small \& Melnyk, 2006). Mothers with increased anxiety levels are inhibited from effective parenting during their child's hospitalization. Maternal state anxiety was found to be the most significant predictor of children's later aggressive and hyperactive behavior for as long as six months following PICU hospitalization (Small \& Melnyk, 2006). Melnyk and Feinstein (2001) found high maternal anxiety led to high anxiety levels in the child. Literature will be reviewed on children's developmental understanding of illness; the PICU environment, its stressors, fears, and memories; and 
the child's response to the PICU hospitalization: factual and delusional memories, delirium and ICU psychosis, and post-traumatic stress.

\section{Children's Understanding of Illness}

Children's understanding of illness, its causes, prevention, and treatment differs significantly at various stages of development from early childhood through adolescence. Early research found children's understanding of illness follows a developmental sequence with significant increases in knowledge with increasing age (Bibace \& Walsh, 1980). Later research, with a more sophisticated approach, indicates younger children are more competent in their understanding than suggested by earlier studies (Kalish 1996, 1997). Both children and adults may revert to immanent justice and folkloric explanations for causes of illness and death when faced with stressful and uncertain situations (Ramen \& Winer, 2002). The specific meaning of an illness to a child may vary with the nature of the illness, the child's ability to understand it as a phenomenon, previous experience, the family situation, and the child's age (Bares \& Gelman, 2008; Myant \& Williams, 2005; Peltzer \& Promtussananon, 2003; Piko \& Bak, 2006; Williams \& Binnie, 2002). The child's health status has an effect on their perception of illness creating some discrepancies in the perceptions of healthy, hospitalized, and chronically ill children (Koopman et al., 2004; McQuaid, Howard, Kopel, Rosenblum, \& Bibace, 2002; Veldtman et al., 2001). Results are equivocal regarding chronically ill children's perception of illness. Paramount to expanding the state of the science relating to children's understanding of illness is the need to examine these differences though the lens of healthy and ill children. 
Several seminal research studies in healthy children have formulated the theoretical underpinnings for children's understanding of illness. The traditional, developmentally naive child concept of illness approach follows Piagetian cognitive theory incorporating general stages of cognitive development across all domains with cognitive structure being content-independent and domain-general (Zhu \& Fang, 2000). Bibace and Walsh (1980) conducted research designed to further refine their earlier prepilot and pilot study results. Children $(N=72)$ were placed into one of three age groups corresponding to Piaget's developmental stages: 4-year-olds representing prelogical, 7year-olds representing concrete logical, and 11-year-olds representing formal logical stage. There were 12 boys and 12 girls in each of the groups. Using the Concept of Illness Protocol, children were asked 12 questions about common illnesses (e.g. cold, heart attack, measles, headache, and pain) as well as the child's explanations of illness.

Bibace and Walsh (1980) results demonstrated consistency with Piagetian stages of cognitive development but also revealed two subtypes within each of the major Piagetian categories, and one category of "incomprehension" characterizing the very youngest subject in the pilot study. Consecutive, developmentally ordered subthemes included phenomenism and contagion in Piaget's preoperational stage; contamination and internalization in the concrete operations stage; then physiologic and psychophysiologic in the formal operational stage.

Children in Piaget's preoperational stage ( 3 to 6 years) are unable to separate themselves from the world around them, explaining illness through their senses and/or experiences. Children in this stage are egocentric and overly swayed by their immediate needs. Children at the most developmentally immature stage of phenomenism identify the 
cause of illness through external influences occurring at the same time. These occurrences may not be spatially close to the child. The next stage of contagion has the importance placed on the physical proximity of the person and the cause. Preoperational children frequently conceptualize causes of illness through magical thinking (Youngblut, 1994).

Children move into the stage of concrete-logical reasoning between 7 and 10 years of age. Piaget describes this stage as having a major developmental shift where children can differentiate between themselves and others and the ability to distinguish what is internal versus external (Bibace \& Walsh, 1980). Two explanations for causes of illness are characteristic of this stage: contamination and internalization (Bibace \& Walsh, 1980). Children in the contamination stage now understand the cause of illness is an external person, object, or event, but contact is required for illness to occur. Older children in the internalization stage recognize illness as inside the body yet the cause may be external, usually a person or object. Internalization of the illness is now linked to internalization of the cause, usually through inhaling or swallowing (Bibace \& Walsh, 1980; Youngblut, 1994).

The formal operations stage describes children from approximately 11 years as being able to think more abstractly and is capable of manipulating concepts. There is now a great differentiation between the self and others. Younger children in this stage view the cause of an illness as physiologic, understanding the cause of the illness may be triggered by external sources with the illness within specific internal organs and/or processes. These children can frequently describe the events leading to the illness development in a sequential manner. The most mature understanding of illness is 
represented through psychophysiologic explanations. Older children in this stage are capable of recognizing the addition of psychologic causes for illness; a person's thoughts or feelings may be responsible for the cause of illness (Bibace \& Walsh, 1980; Youngblut, 1994).

The Bibace and Walsh (1980) explanation of illness varied as a function of the child's developmental status with more mature explanations given by older children. Later research, with a more sophisticated approach, found that children are more competent than Piaget believed (Kalish, 1996, 1997).

\section{Healthy Children}

In a randomized controlled trial, Williams and Binnie (2002) conducted a study in Great Britain examining 60 children's concepts of contagious illness (chickenpox and colds), non-contagious illness (asthma and cancer), and injuries (scraped knee and broken arm) and understanding of causal factors, cause and symptom time line, and recovery factors. All children were individually interviewed during the pre-test using fixed-choice questions relating to the three ailment types examining the dimensions of illness causality, time from cause to effect, and recovery factors. Cartoons illustrating each question were used to maintain the child's interest. Responses were scored by research personnel on a 1- to 4-point scale with higher numbers indicating more advanced levels of response. Following pretest interviews, half of the 30 children in each age group (3and 4-year-olds vs. 6- and 7-year-old) were randomly assigned to intervention or control conditions. The intervention occurred one week after the pretest with the group receiving indirect experience through vignettes, factual information through story books, and guided group discussions relating to illness while the control group received no 
interventions. One week following intervention completion, all children were independently interviewed using the same materials and procedures as the pre-test.

Pre-test results indicated a significant age differences in understanding between 3/4- year-olds with less mature responses and the 6/7-year-olds with more mature responses for the majority of items indicating increasingly biological understanding. Responses for items on chickenpox time, chickenpox recovery, cold time, and asthma time showed no maturation in children's understanding of time course factors between 3 and 7 years. Pre-test data also indicated that injury knowledge was more advanced in both ages than knowledge of contagious and non-contagious illness. This may be explained by direct experience or because minor trauma is easier to conceptualize. Little evidence was found indicating children understood contagion or germ theory but instead over-extended the concept of contagion to non-contagious processes. Significant age improvement in knowledge between 4 and 7 year olds was noted in almost all of the items indicating increasing biological understanding of illness and injury with increments in age (Williams \& Binnie, 2002).

Overall improvement was found in global difference scores (total post-test minus total pre-test scores across all processes and ailments) as a function of age and group assignment. The 4-year-olds showed the most improvement and children in the intervention group showed the most improvement from pre- to post-test regardless of age. Four-year-olds demonstrated greater levels of post-test improvement compared to the 7year-olds implying the younger child is ready and able to learn about illness concepts. Results indicated children's difficulty in understanding time course factors as seen in lack of change in response with age for chickenpox time, chickenpox recovery, cold time, and 
asthma time. Introducing the younger child to health education may facilitate the development of a firmer basis for understanding illness. Study results indicated the intervention group had greater knowledge than the control group; however, study design prohibited determining whether a specific intervention component, the story or group discussion or the combination of the two, was associated with conceptual advancement. Children's responses were classified as improvement, no change, or regression from preto post-test. Significant associations were found between age and levels of improvement for cold recovery, asthma cause, asthma recovery, cancer cause, scraped knee recovery, and broken arm cause with more improvement and less regression seen in the 4-year-olds at post-test (Williams \& Binnie, 2002).

This study highlighted the importance of providing children with correct factual information and facilitative peer discussions about illness to increase their knowledge base. The study did not provide correlations of specific interventions with conceptual advancement. Whether it was the story information or group discussion that increased the child's understanding could not be determined. Additionally, data points between interventions and post-test are not clear creating a possibility of children's knowledge advancing through other methods. The small sample was also a study weakness (Williams \& Binnie, 2002).

Peltzer and Promtussananon (2003) used semi-structured interviews to investigate 5- and 9-year-old $(N=60)$ South African children's understanding of health and common conditions (colds, broken arms, chicken pox, and AIDS) and their exposure to learning about health or sickness. Children were recruited through random sampling from a rural, primary school in South Africa. The interview design was adopted from an 
earlier New Zealand study in English. In addition to the open-ended questions, the interview included measures of well-being using a 7-point rating scale from 1 "extremely happy" to 7 "extremely sad"; six questions relating to illness knowledge; and if they thought there was anything they could do to stay healthy or well using a 4-point rating scale ranging from 1 for "lots of things" to 4 for "nothing" with subsequent questions about strategies they could use to prevent a cold, broken arm, chicken pox, and a cold.

The age groups were evenly distributed. The younger group had more females (57\%) than the older more males (37\%). Results indicated older children (80\%) had more exposure to health education than the younger children (57\%) and were better able to discuss objective disease symptoms than younger children. Parents were described as the source of information for colds, broken arms, and chicken pox, and radio or television the source for AIDS in both age groups. All 60 children described contamination as a cause of colds, and most understood car accidents could cause a broken arm; however, the majority of children in both age groups did not know the cause of chicken pox. Children frequently identified contamination and contagion as illness causes but had limited understanding of germs. More children (63\%) identified strategies for staying healthy than younger children (37\%). Most children knew strategies to avoid broken arms and about half knew strategies to avoid chicken pox and AIDS. No differences were found between the two age groups regarding causes of illness with the exception of AIDS; older children identified contagion as a cause of AIDS more frequently than the younger children. Results indicated the children's understanding of AIDS followed the same developmental sequence as reported for understanding of general physical illness. 
Increased knowledge in the older child could be due to the effects of increased TV and radio exposure (Peltzer \& Promtussananon, 2003).

Study limitations included the small sample, small range in child ages, and the sample being drawn from one rural area. Additionally, the data was not analyzed quantitatively but reported only through descriptive or frequencies. Parametric testing would have provided stronger information (Peltzer \& Promtussananon, 2003).

To develop a more comprehensive picture of children's understanding of illness and injury, Myant and Williams (2005) conducted semi-structured interviews using introductory questions and six vignettes to elicit general definitions of health and personal experiences with 83 children from state-run and private nurseries in Glasgow. The sample was determined using power analysis and was comprised of four age groups: 4/5-year-olds $(n=20), 7 / 8$-year-olds $(n=20), 9 / 10$-year-olds $(n=21)$, and 11/12-yearolds $(n=22)$.

Participants were individually interviewed with two introductory questions asked to generally define health and illness. This was followed by six vignettes using child characters with different illnesses: contagious illnesses (colds and chicken pox); noncontagious illnesses (asthma and toothache); and injuries (bruise and broken leg). A cartoon drawing of each of the characters was shown with the vignette to keep the child's interest. Questions about each illness were then asked inquiring about illness definition, causality, multiple causality, prevention, time course, and recovery. Definition of illness was categorized into "don't know", psychological, behavioral, and symptoms. Results indicated general definitions of illness and health as well as knowledge of specific illnesses was more accurate and sophisticated in older age groups. Behavioral definition 
was the most preferred category for the 7/8-year-olds and the 11/12-year-olds; 4/5-yearolds “don't know"; and 9/10-year-olds preferring absence of symptoms. Significant developmental improvements between age groups was also seen with knowledge of illness causality increasing with age for chicken pox, cold, and toothache; no age differences were found for bruise, broken leg, and asthma. Developmental trends were also reported with prevention strategy scores, time course, and recovery, increasing across the age groups for each illness. Asthma scores were lower than all other illnesses in the three scores possibly explained by the lower amount of experience with asthma than the other illnesses. Findings also indicated children are able to refer to a biological framework of understanding as young as $4 / 5$ years without having specific causality mechanisms. The 11/12-year-olds indicated beginning understanding of incubation period and recovery time involved in contagious illness. Children as young as 4/5 demonstrated some understanding of contagion and causality; however, results indicated children's linking of causality with contagion was not accurate until approximately age 9. The 4/5-year-olds may not have been able to fully describe their understanding due to their limited verbal ability.

Piko and Bak (2006) investigated Hungarian 8- to 11-year-olds' $(N=128)$ concepts of health, illness, health promotion, and disease prevention using draw and write methodologies. The researchers' hypothesized Hungarian children would differ from Western children due to differences in social and cultural contexts. Children were recruited from two primary schools in Hungary reflective of average socioeconomic and health literacy backgrounds. One class from each of Grades 3, 4, and 5 from each of the schools were selected with data provided by children present in the selected class the day 
the research was conducted. Children were given pre-prepared questionnaires containing questions relating to their age, sex, health beliefs, health promotion, and disease prevention. Responses were drawn or written by the children then discussed with them to determine their meanings. The majority of children gave sophisticated biomedical and holistic health definitions, describing contagion and contamination processes as causes of illness. Some identified environmental factors (e.g. bad air) as causes of illness. Many of the preadolescent children cited risky behaviors for causing illness with most of the children identifying healthy lifestyle to maintain health and prevent disease.

Preadolescent children identified sports as a preventive health behavior. More drawings were provided in reply to the question asking about illness causality than to the question asking about health definitions.

Corresponding to findings by Myant and Williams (2005), drawings relating to disease causation indicated children may not have enough cognitive ability to express their views. Most children emphasized healthy lifestyle for health maintenance and disease prevention with behaviors identified such as sports activities, nutrition, and avoidance of harmful habits. Cleanliness was identified as a method for disease prevention and preadolescent boys indicated engaging in sports as a preventive health behavior. Healthy environments were identified as important for health promotion and disease prevention. Study results indicated Hungarian children have considerable knowledge about health, illness, and disease risks that is similar to that of Western children. Children expressed a strong biomedical and holistic approach to health emphasizing harmony between themselves and the environment (Piko \& Bak, 2006). 
Many cross-cultural similarities were found in children's concepts of health indicating a more universal phenomenon with little cultural influence.

Bares and Gelman (2008) recruited 36 children from U.S. Midwestern preschools and after-school programs and measured their knowledge of six dimensions of cancer and colds (prognosis, internal, course, contamination, contagion, and cause) using their own 34-item questionnaire; Cronbach's alpha was .61. The same instrument was used to test 25 adults (13 males; 12 females) recruited from a University psychology class to provide age comparisons. Questionnaires consisted of two sets of 17 questions (cancer and colds) with scenarios depicting hypothetical ill characters (matched to the participant's gender) with either a yes/no or forced choice response. Researchers read the scenarios aloud to the children. Children were individually interviewed during their preschool hours or during after-school hours. Adults were interviewed as a group during their class with questionnaire completion in paper and pencil format.

The sample included fourteen 5-year-olds, fourteen 7-year-olds, and eight 10year-olds. Results demonstrated a developmental progression of illness knowledge with each age group possessing distinct beliefs and ideas regarding illness and cancer. The 5year-old children attributed cause for both cancer and colds to contagion and thought they were similar in length and severity; however, they had a high understanding of the internal nature of conditions. The 7-year-olds were beginning to differentiate between a cold and cancer regarding duration, severity, and transmission. The 10-year-olds identified an understanding of the severity of cancer, believed it was not transmitted through contagion, and did not apply contamination to cancer or colds. These 
developmental changes may be explained by increasing education from external sources as the child ages and greater cognitive processing skills (Bares \& Gelman, 2008).

Results of this study describe a developmental progression of understanding cancer and colds from childhood to adulthood. By adulthood, participants were able to distinguish between the two illnesses on five of the six dimensions. The study, however, does not identify which variables may account for the conceptual changes between the age groups. Developmental changes may be explained through external sources such as peers and social networks as well as school education. The researcher-developed questionnaire reflected Western thinking therefore may not be generalizable to other cultures (Bares \& Gelman, 2008).

Zhu, Liu, and Tardif (2009) investigated the causal explanations of illness in 90 Chinese preschool children and 30 Chinese college students (range 19-22 years) using interview techniques. Thirty children were in each of the three age groups of 3-, 4-, and 5-year-olds. Half of each group was from university-run kindergartens with highly educated parents and the other half from a suburban kindergarten with less educated parents. Participants were asked why one gets sick; children were individually interviewed in quiet kindergarten rooms and adults responded in writing in group sessions.

Responses were coded into five mutually exclusive categories identified for explanations of illness causality were psychogenic, biological, behavioral, symptomatic, and irrelevant with differences between adults and children. Adults most frequently gave biological explanations with some mention of psychogenic causes while children most frequently identified behavior for causes of illness. Increasing biological explanations 
was related to increasing age of the children. Differences in descriptions of illness were seen between the two socioeconomic (SES) groups. Biological explanations for illness in the 5-year-old group was given by almost half of the higher SES group but only oneeighth of children in the lower SES group. Most of the lower SES 5-year-olds provided behavioral explanations. The majority of participants incorporated folk biology such as kicking off the quilt while sleeping and named aspects of daily activities as affecting illness susceptibility. Older children identified biological terms for illness causality such as germs and bacteria; however, similar to Western cultures, they did not understand pathophysiology. Children from higher SES groups offered more biological causes for illness than adults in lower SES groups reflecting effects of personal experience, direct observation, and especially education. Traditional concepts of Chinese medicine tying negative emotions to illness influenced adults; however, Chinese children did not mention emotional causes for illness. Contrary to other studies, immanent justice was not mentioned by the children as a cause of illness. Consistent with results by Raman and Winer (2002), findings demonstrated both children and adults used biological and folkloric (e.g. wind, cold) explanations of illness (Zhu et al., 2009).

\section{Ill Children}

Using semi-structured interviews, Veldtman et al. (2001) studied 63 children from England between the ages of 7 and 18 years with a variety of congenital heart defects regarding their understanding of their own defect in relationship to age, sex, or complexity of heart disease. Children were identified from a Heart Center database and were invited to participate by letter or telephone prior to a routine outpatient visit. Adolescents were interviewed in an adolescent decorated, quiet room in the outpatient 
area without the presence of their parents. Younger children were given the choice of having their parents present with interviews conducted in the home when possible. The interviewer was informally dressed. A conversational approach was used in asking the name of the child's defect, what was wrong with their heart, how long they thought they had the condition, and how was their heart likely to affect them in the future. Knowledge related to name of diagnosis and perceptions of illness were measured on a three-point scale constructed from hospital cardiologists' criteria: wrong/poor explanation, partial explanation, and good explanation.

Results indicated only 14 (22\%) of the children knew their diagnosis by name; 23 $(36 \%)$ had a poor understanding of the nature of their cardiac disease, $21(33 \%)$ a partial understanding, and $19(30 \%)$ a good understanding. Controlling for severity of cardiac disease, no significant relationship was found between level of understanding and the child's age, sex, or cardiac complexity. Understanding of the illness time line improved with increasing age. Those with good understanding were a mean of 15 years old; those with poor understanding were an average of 10.5 years old indicating older children had more developed perceptions about the effects of their illness. Decreased illness knowledge may result from subtle developmental delays occurring as a result of congenital heart defects (Veldtman et al., 2001).

Recognizing a child's cognitive level of reasoning about illness is separate from, but related to their factual knowledge, McQuaid, Howard, Kopel, Rosenblum, and Bibace (2002) interviewed 104 New England children with persistent asthma ages 7 to 16 ( $M=$ 11.7 years) and 90 of the children's parents about their factual knowledge of asthma, causal reasoning regarding headache and asthma, and strategies for prevention. Families 
were recruited through emergency department, asthma education participation, and asthma camp attendance records. All children were taking regularly prescribed asthma medications administered regularly by inhaler.

Individual interviews were conducted for children and their parents. Asthma severity was measured using the National Heart, Lung, and Blood Institute criteria with parents providing information regarding how they determined disease severity and medications prescribed. A structured questionnaire developed for earlier studies was used to obtain descriptive information regarding the child's functional impairment from asthma (e.g. school days missed). A pediatric asthma specialist reviewed those findings and categorized the severity of the children's asthma: 1 (mild intermittent asthma) to 4 (severe persistent asthma). Basic factual knowledge was measured using the Asthma Knowledge Questionnaire (AKQ) with parent and child versions. Children's and parent's concepts of illness relating to headache and asthma were measured using the Concepts of illness Interview which included questions regarding illness causality, prevention, and medications for headaches and asthma. The participant's maximal level of conceptual reasoning was determined (McQuaid et al., 2002).

All children were recruited based on their need for daily asthma medications resulting in very few with mild intermittent asthma (6\%). The majority of the children had mild persistent (55\%) followed by moderate persistent (33\%), then severe persistent asthma (6\%). Insufficient information was collected on five children resulting in the inability to categorize them. Parent participants were primarily mothers $(n=83) ; 41 \%$ of the children were female and Caucasian (64\%). Coders were blinded to child age, gender, and disease severity (McQuaid et al., 2002). 
Responses were scored from one to six according to level of causal reasoning regarding illness and not on factual accuracy; more sophisticated reasoning was given higher scores. Responses per participant were summarized using the mode for the most frequent response and the highest response given, indicating the highest level of reasoning expressed. Results found basic asthma knowledge was significantly associated with age but not to children's developmental concepts of headache. Children's reasoning about headache was most often classified at the Contamination level while asthma was at the higher Internalization and Physiological levels. No association was found between children's reasoning and basic knowledge relating to asthma and that of their parents even after controlling for the child's age. Parents and children, regardless of age, demonstrated higher causal reasoning of illness for asthma compared to headache with results suggesting children's reasoning regarding headache was predominantly at the Contamination level (61\%) and asthma at the higher Internalization (35\%) and Physiological (33\%) levels. The majority of parents responded at the Internalization level for headache (53\%) and asthma (42\%); parents reported at the higher Physiological stage for asthma and (36\%) and headache (6\%). These results suggest more experience and education may facilitate disease knowledge. Parents of children with moderate persistent asthma were found to have the highest mean scores for knowledge levels, followed by mild intermittent and mild persistent, and the three parents of children with severe asthma demonstrated the lowest scores. This could be explained by levels of stress and anxiety interfering with the ability to learn and retain knowledge (McQuaid et al., 2002). No association was found between children's and their parents' basic knowledge and reasoning regarding asthma. Children and their parents gave more 
sophisticated explanations for asthma exacerbations than headaches consistent with other research indicating children have higher concepts of illness regarding common experiences such as cold than illnesses they may not have experienced, such as AIDS (Peltzer \& Promtussananon, 2002). Findings supported the researchers' hypothesis that repeated experience and education lead to more sophisticated concepts.

Koopman, Baars, Chaplin, and Zwinderman (2004) compared the development of causal thinking about illness and illness-related concepts (pain, becoming ill, and going to the doctor) in 80 children (6 to 12 years old) with diabetes mellitus (DM) $(M=8$ years, 9 months; $S D=1.9$ years) and 78 healthy classmates ( $M=8$ years, 8 months; $S D=2$ years $)$ from the Netherlands. The role of anxiety, locus of control, and family- and schoolfunctioning were measured to determine the influence of stress on the development of causal thinking about illness. Pediatrician's from nine hospitals randomly selected children with DM for recruitment. Average age of diabetes diagnosis was 5 years, 2 months ( $S D=2$ years, 4 months); children had been diagnosed between 6 months and 8 years ( $M=3$ years; $S D=2$ years, 4 months). More than $55 \%$ of the children were female.

The children were interviewed during school hours about disease etiology, treatment, and prevention. Responses were scored using the developmentally ordered Bibace and Walsh Illness Category System. Measures included cognitive function (Floating and Sinking of objects), locus of control questionnaires, a family relation test. Children were asked to tell a story of their choice measuring anxiety levels on the Gottschalk and Gleser content analysis of verbal behavior. Raters were blinded with respect to the child's information. Responses were scored for their conceptual 
sophistication without regard to factual accuracy (Koopman, Baars, Chaplin, \& Zwinderman, 2004).

Boys scored higher than girls in cognitive function with higher scores significantly correlated with greater age. No differences were found in scores between healthy children with DM. Children with DM had significantly higher Interview Illness scores than the healthy children. Family and school function were not related to stages of reasoning about illness; however, healthy children showed more positive feelings toward their mothers than those with DM. Children with DM experienced more parental over protection. Consistent with developmental stage sequences (Bibace \& Walsh, 1980), understanding of illness-related concepts matured with increasing age. Statistical significance was not found for relationships between children's thinking about illness and anxiety, locus of control, and family- and school-functioning. Higher levels of anxiety were found with older children than younger children. The only association found between anxiety and the Illness Interview Sum score was found in children with DM who reported more feelings of shame. Intellectual development was not related to children's illness causality concepts. Level of illness concepts sophistication was not affected by gender and SES. Concepts and causes of illness followed the same sequential developmental stages described by Bibace and Walsh (1982) with a systematic progression in understanding illness-related concepts with age (Koopman et al., 2004). In summary, children's development of understanding illness is hierarchical with subjective, intuitive, and naive beliefs typically associated with younger children at the bottom, and the most advanced, logical, and scientifically-based thinking at the top (Bares \& Gelman, 2008; Myant \& Williams, 2005; Peltzer \& Promtussananon, 2003; 
Piko \& Bak, 2006; Williams \& Binnie, 2002). Definitions of illness were found to be more polarized with age (Myant \& Williams, 2005). Contrary to Piagetian models, where newer stages of development replace earlier stages, coexistence theories allow for movement between stages (Ramen \& Winer, 2002). Two studies (Ramen \& Winer, 2002; Zhu et al., 2009) found children and adults used folkloric and biological explanations for illness and resort to immanent justice (Ramen \& Winer, 2002) when faced with uncertainty.

Multiple variables may affect children's perceptions of illness. Repeated exposure and education was found to increase children's understanding of illness with significant developmental changes noted as young as age 4 to 5 (Bares \& Gelman, 2008; Peltzer \& Promtussananon, 2003; Piko \& Bak, 2006; Williams \& Binnie, 2002; Zhu et al, 2009) but the ability to link contagion and causality was not evident prior to the age of 9 (Myant \& Williams, 2005; Peltzer \& Promtussananon, 2003; Williams \& Binnie, 2002). Zhu et al. (2009) found higher SES and educational groups correlated with increasing biological explanations than lower SES groups. Many children expressed understanding of contamination and contagion and stated that germs may play a role in illness; however, their understanding of germ theory was limited (Peltzer \& Promtussananon, 2003; Piko \& Bak, 2006; Williams \& Binnie, 2002).

A major limitation of the studies examining children's understanding of illness was the inclusion of a single cultural context relevant to that study decreasing the generalizability to other cultures or contexts. Additionally, many of the studies did not identify SES therefore results may not be generalizable to lower socio-economic groups. Questionnaires developed in many of the studies reflected 
Western thinking only creating limitations to generalizability to other cultures. Many instruments/questionnaires were researcher-developed with unclear psychometric properties. The lack of longitudinal design in many of the studies makes it difficult to reflect the developmental progression of children over time.

Many of the studies used interview techniques; however, children may lack the cognitive ability and skills to verbalize their understanding or perceptions. The use of drawing has been shown to be an effective method to investigate children's understanding of health and illness at different levels of cognitive development. The method has drawn criticism from researchers addressing methodological, analytical and ethical faults with the technique. Accuracy of data analysis has been questioned based on interpretation concerns, especially when drawings are used as primary data. Children's drawings, especially in conjunction with other methodologies, can serve as a powerful method of investigating children's concepts (Piko \& Bak, 2006).

\section{PICU Environment: Stressors, Fears, and Memories}

\section{Stressors}

Miles and Carter (1982) classified sources of stress experienced by parents of children in the PICU as personal, environmental, and situational aspects of the PICU environment; intensity of PICU personnel; and presence of other critically ill children, families, and caregivers. These factors are all alien and stressful for shocked children, parents, and families (Namachivayam et al., 2010). The complex PICU environment exposes children to many phenomena with which they have little or no experience and to 
activities that occur with minimal or no warning. Children are forced to assimilate the experience within a very short period of time (Vanek, 1979).

In an early study of the types and dimensions of stressors most affecting children in PICU, Tichy, Braam Meyer, and Rattan (1988) investigated children $(n=10)$ between 7 and 17 years $(M=13.6$ years) and their parents' ( 7 mothers, 2 mother-father pairs, and 1 father) perceptions of stressors most affecting their children in the PICU. Semistructured interviews developed by nurse researchers and clinical specialists were conducted 48 hours after the child was discharged from PICU. Coding categories were developed from parent and child reports of stress. Cumulative frequency of stressor categories and parent and children responses were compared. Results found children indicated the worst stressors were invasive procedures causing pain and discomfort (44\%), environmental (26\%), social (16\%), and psychological (13\%). There were similarities between child and parent report of stressors; however, stressor frequencies between them were statistically different. Several parents identified nurse presence as a comfort to the child while the child considered it a stressor. Children also stated the parents' expressions caused additional stress while none of the parents interviewed were aware of this.

Admission to the PICU affects children and their families far beyond their physical well-being. The trajectory and treatment of critical conditions expose children to a wide range of extreme stressors which include painful, frightening and invasive procedures; distressing environmental events; threat to life and physical integrity; ventilators; intravenous pumps; bright lights; strangers; and other sick children (Dow et al., 2012); respiratory insufficiency; separation from families; loss of control; and 
delirium with potential associated psychotic experiences (Davydow et al., 2010).

Although some of the equipment used in the child's care maintains life support, younger children may view the large, noisy machines as extensions of themselves and believe all the machines control their bodily functions (Vanek, 1979).

Noise.

High noise levels in the ICU are a serious concern for both patients and health care providers. The causes of noise are primarily environment (linked to technology), the structural unit characteristics (e.g. flooring, ventilation), and the social interactions of the people in the unit. There are multiple sources of auditory stimuli in the ICU with patients having little or control over it. Any sudden, unexpected sounds cause extra stress to patients who are already stressed (Giusti \& Piergentili, 2009).

Researchers developed a weighted unit of sound intensity known as the Aweighted decibel, or $\mathrm{dB}(\mathrm{A})$ scale to accurately reflect the ear's sensitivity to sound intensity over the range of audible frequencies; an increase of $10 \mathrm{~dB}$ (A) results in a perceived doubling of loudness (Cmeil, Karr, Gasser, Oliphant, \& Neveau, 2004). The Aweighting is most commonly used intended to approximate the frequency response of our hearing system. It weights lower frequencies as less important than mid- and higherfrequency sounds. C weighting is also reported measuring a nearly flat frequency response with decreases of high and low frequency extremes (Berglund, Lindvall, \& Schwela, 1999, p.22).

The World Health Organization [WHO] (Berglund et al., 1999), the International Noise Council [INC] (Elander \& Hellström, 1999), and the Environmental Protection Agency [EPA] (Environmental Protection Agency, 1999) have set recommended 
maximum allowable standards for allowable noise for hospital patient rooms. Recommendations differ based on the agency ranging between 35 and $45 \mathrm{dBA}$ for daytime and 20 to $30 \mathrm{dBA}$ for night. The American Academy of Pediatrics (AAP) recommended monitoring sounds in the NICU and within incubators with noise levels greater than $45 \mathrm{Db}$ a concern (1997).

Research indicates noise has cardiovascular and physiological effects which could affect healing and mental health. It causes sleep disturbances which may exacerbate confusion and ICU-related delirium. Excessive noise can lead to long-term hearing effects in adults, neonates, and fetuses (AAP, 1997; Busch-Vishniac, West, Barnhill, Hunter, Orellana, \& Chivukula, 2005). An early study by Long, Lucey, and Philip (1980) found hypoxemia occurred in infants with sudden loud noises $(\sim 80 \mathrm{dBA})$.

Multiple studies conducted in Adult ICU and NICU populations report sound levels in those areas exceed the recommendations. Busch-Vishniac, West, Barnhill, Hunter, Orellana, and Chivukula (2005) described higher than recommended noise levels within Adult ICUs increasing since the 1960s by an average of $0.38 \mathrm{dBA}$ (day) and 0.42 (night) and are increasing linearly. Some common ICU noise levels are telemetry alarms levels at over $70 \mathrm{~dB}$, electrocardiogram alarms at $75 \mathrm{dBA}$, intravenous infusion alarms between $44-80 \mathrm{dBA}$, measured infusion pump alarms at $61 \mathrm{dBA}$, and monitor alarms at 68 dBA (Akansel, Neriman, \& Kaymakci, 2008; Taylor-Ford et al., 2008).

Darbyshire and Young (2013) recorded day and night time sound levels over two weeks in five adult ICUs in England. Sound levels were collected using two portable sound level monitors with microphones in each ICU which continuously recorded. Monitors were placed centrally in the unit or adjacent to the central station and adjacent 
to the patients head. Average sound and peak levels were recorded every minute.

Average sound levels always exceeded $45 \mathrm{dBA}$ and exceeded between 52 and $59 \mathrm{dBA}$ in individual ICUs $50 \%$ of the time. All ICUs recorded peaks above $85 \mathrm{dBA}$, up to 16 times at night and more frequently during the day. Findings indicated WHO guidelines could only be achieved in a side room by switching all equipment off. Lawson et al. (2010) emphasized the importance of measuring mean levels as well as peak sound levels finding large discrepancies in their adult ICU study reporting mean sound pressure levels in the patient's room below $45 \mathrm{dBA}$ yet peak levels were greater than 85 .

A recent NICU study by Matook, Sullivan, Saunders, Miller, and Lester (2010) reported higher noise levels in day shifts than night shift and significantly higher levels on weekdays than weekends. The average sound level varied from 49.5 to $89.5 \mathrm{dBA}$ ( $M$ $=85.15 \mathrm{dBA})$ with peak sounds ranging between 66 and $139 \mathrm{dBC}(M=134.5)$. Similar findings were reported by Williams, van Drongelen and Lasky (2007) comparing noise levels in different levels of care (isolation, level II, level III) within two separate hospital NICUs. Sound levels were measured for eight days using a dosimeter with results indicating higher than recommended levels were found more than $70 \%$ of the time in all the units. Kellam and Bhatia (2008) measured sound in two rooms of a level III NICU using a sound level meter placed at a 45 -degree angle within $15 \mathrm{~cm}$ of the infants' ear. Measurements were collected over a four week period between 7 a.m. and 10 p.m. at patient bedsides, during shift change, around oscillator ventilators. Lowest levels were reported in units with "feeder-grower" babies. The most intense frequencies occurred at change of shift, taking vital signs and performing assessments, preparing feedings, and participating in physician rounds. Bedside readings varied according to proximity of 
windows, outer walls, and direction of mounted monitors; infants on ECMO were exposed to $16000 \mathrm{~Hz}$ (equivalent to $50 \mathrm{~dB}$ on an A-weighted scale). High noise levels were also reported with nurse communication particularly in an area separated by columns. For speech to be heard, nurses had to shout over the background noise. Similar to adult and NICU findings, high noise levels were found in children's ICU settings. Exceeding the recommended highest noise levels of 45 dBA (WHO, 1999), noise levels in PICU studies were measured at $70 \mathrm{dBA}$ during the day and $59 \mathrm{dBA}$ at night, with maximum levels reaching $120 \mathrm{dBA}$ (Al-Samsam \& Cullen, 2005; Carno \& Connolly, 2005; Morrison, Haas, Shaffner, Garret, \& Fackler, 2003). A Canadian study by Milette and Carnevale (2003) was conducted to evaluate the level of noise in an uncarpeted PICU and compare it to recommendations made by internal agencies. The layout of the room was an open design with a capacity of eight beds in the open area, four single bed isolation side-rooms attached to the open area, and an unattached step-down unit of six beds. Four beds were on each side of the open layout with a central station located in the center of the room. Averages of 15 medical persons were in the unit at one time, mostly during procedures, or rounds. Parents were permitted to stay 24 hours a day resulting in at least one parent per bedside during the study. Noise levels were monitored at each of the four corner beds, the central station, and one side-room using the Precision Sound Level meter and Analyzer. Additionally, comparisons were made for change-ofshift report as well as contrasts between night and day shift.

The mean PICU noise level was found to be significantly higher $(p<0.000)$ than the highest recommended level of $45 \mathrm{dBA}$. Hemodialysis recorded the highest level of noise (63 dBA) and the ECMO patient had the largest variation in noise levels noted. 
The highest peak recorded was with the high-frequency ventilation recording (peak $=102$ dA) and a cardiac patient recorded the most number of peaks above $80 \mathrm{dBA}$. This was the busiest patient of the sample, having two nurses and a physician continuously at the bedside. Dayshift was found to be significantly noisier than the night shift $(p<0.000)$ and change of shifts were significantly noisier than general level of PICU noise (Milette \& Carnevale, 2003).

Carvalho, Pedreira, and de Aguiar (2005) found similar findings in a 10 bed Brazilian PICU. Sound pressure levels (dBA) were measured for 24 hours during a 6-day period with noise recording equipment placed in the PICU nursing station, access corridor, isolation rooms, and two open wards with three and five beds. The basal noise level ranging between $60-70 \mathrm{dBA}$ with peak hours of noise noted between $10 \mathrm{a} . \mathrm{m}$. and 4 p.m. The highest noise levels were found in the nurses' station and the corridor leading to the PICU. A basal noise level of $80 \mathrm{dBA}$ with a maximum level of $120 \mathrm{dBA}$ was found during the admission of a post-cardiac surgery patient originating from communication between the PICU staff, surgeons, and anesthesiologists. Variations demonstrate differences in intermittent and constant noise levels in a PICU. Equipment noise was most significant from the triggering of certain alarms and during the normal operation of the mechanical ventilator, followed by the 'bip' of the cardiac monitors.

\section{Lighting.}

Critical care units are typically bright with harsh, artificial lighting which is rarely turned off or dimmed fearing this will compromise patient care. Many ICUs are designed without windows or have the bed positioned to prevent the child's view of the window. The artificial light used is predominately fluorescent producing visual fatigue and 
headaches (Fontaine, Briggs, \& Pope-Smith, 2001). The continuous light experienced by ICU patients not only disrupts the circadian rhythm but also drops melatonin levels with the possibility of total cessation of melatonin production. Decreased levels of melatonin can cause impairment in sleep patterns and delirium in critically ill patients. Disruptions in the circadian rhythm also can lead to disorientation, delirium, or ICU psychosis in severe cases (Fontaine et al., 2001; Starkweather, Witek-Janusek, \& Matthes, 2005).

Studies relating to effects of PICU lighting were not found; however, studies conducted in the NICU population suggest unnatural, continuous lighting as well as the lack of regular light/dark cycles adversely affects these babies. The AAP and the American College of Obstetricians and Gynecologists (2007) as well as the Committee to Establish Recommended Standards for Newborn ICU Design (2012) recommend that ambient light level at each infant bedside be adjustable from 10 to $600 \operatorname{lux}(10 \operatorname{lux}=$ approximately 1 foot-candle) (Lee, Malakooti, \& Lotas, 2005).

Lee, Malakooti, and Lotas (2005) conducted a descriptive, comparative design comparing light reduction capacity of 23 incubator covers in a university medical center NICU. Covers were group into five categories: professionally made $(n=2)$, handcrocheted $(n=10)$, standard hospital receiving blankets $(n=1)$, handmade three-layer quilts $(n=9)$, and blankets made by sewing two layers of flannel together $(n=1)$. NICU ambient lighting levels were measured by placing the light sensor at a point approximating infant eye position in the incubator and covers were described by fabric opacity, percentage of incubator glass covered, and fabric color (white, blue, red/pink, green, and brown) and hue (lightness/brightness or darkness). Light measurements were taken under two conditions: on a sunny day to document the maximum effects of natural 
light and after dark (one hour after sunset) to determine lighting levels. Five measurements were taken to identify the four ambient light levels included in the experimental condition approximating the lowest, highest, average day, and night levels in the NICU. Each of the 23 incubator covers were placed five times for 25 seconds each under each of the four lighting conditions to record light measurements. Covers were removed and repositioned between each of the five trials to simulate actual NICU practices. The five results were then averaged to obtain measurements of light reduction for each cover. Measurements of light levels were assessed in foot-candle (fc).

Ambient NICU daytime light levels ranged from $4.1 \mathrm{fc}$ (44.1 lux) in the areas with little ambient light and no windows to $82.1 \mathrm{fc}$ (884 lux) in the area closest to the windows combined with ambient lighting. Nighttime levels varied from $0.7 \mathrm{fc}(7.5 \mathrm{lux})$ to $58.9 \mathrm{fc}$ (634 lux). Light-reduction levels from the 23 incubator covers ranged from 27.3 percent to 98 percent. The fabrics of the coves varied in the percentage of light reduction they achieved with the opacity of the fabrics ranging between $64.4 \%$ (receiving blanket) to a high of $99.7 \%$ (commercial). No statistical difference was found in light reduction based on the percentage of covered incubator. These results suggest the ambient NICU light levels were well above the recommended standard at peak light periods (Lee et al., 2005).

Lasky and Williams (2009) investigated noise and light environments on extremely-low birth weight $(\mathrm{ELBW})$ premature infants $(N=22)$ enrolled within seven days of birth from a large Texas NICU. The 104-bed unit was constructed with NICU environmental NICU concerns in mind. It was divided in 12 large carpeted rooms with 8 patients per room and 8 isolation rooms with staff work areas separate from patient care 
areas. Each room had three windows allowing natural light. Noise and light were minimized by covering incubators with blankets. Noise dosimeters were placed inside the bed of each newborn within $30 \mathrm{~cm}$ of their ear. Equivalent sound level (Leq) in Aweighted $\mathrm{dB}$ was recorded at 1 -second intervals. Light was measured though light meters placed inside the bed within $20 \mathrm{~cm}$ of the head and recorded in lux at 6-sec. intervals.

Measurements were recorded weekly for approximately 20 consecutive hours until the infant reached 36 weeks' post-menopausal age (PMA) at which time recordings were collected every 2 weeks until 40 weeks PMA, then every 4 weeks thereafter. The final recording was completed for each infant during the week before hospital discharge. Noise levels increased by $0.22 \mathrm{~dB}$ per week although the researchers hypothesized the levels would decrease as the infants stabilized and required less intensive care. These findings were explained by bed type and method of respiratory support with older incubators and ventilators contributing the most to increased noise (Lasky \& Williams, 2009).

Phototherapy greatly affected recorded light levels with 11 of the 251 light sessions recorded occurring during phototherapy administration. Median light levels during phototherapy (809 lux) were approximately 20 times as intense as nonphototherapy recordings (40 lux); these recordings were not included in data analysis. Mean light levels also increased with newborn PMA by 3.6 lux per week. Differences in lights levels were more apparent in open cribs/warmers than incubators. Mean light levels during their NICU stay (26-42 weeks PMA) was 70.6 lux ranging from 21.2 lux to 138 lux; AAP recommended light levels were exceeded $0.64 \%$ of the time (Lasky \& Williams, 2009). 


\section{Sleep Disruption.}

ICUs are complex, fast paced environments with highly skilled personnel providing around-the-clock care for high acuity patients using advanced technology (Walder, Francioli, Mayer, Lancon, \& Romand, 2000). Research has implicated noise, lighting, patient care activities, vital signs, phlebotomy, and medication administration as potential causes of sleep disruption in adult ICU patients (Xie, Kang, \& Mills, 2009). Critically ill children also have been found to have disturbed sleep while in the PICU related to their illness, administering medications, noise, light, and caregiving activities. Sleep disruption impedes somatic growth and cellular repair needed for healing (Carno \& Connolly, 2005).

Carno, Hoffman, Henker, Carcillo, and Sanders (2004) investigated sleep patterns in two sedated and pharmacologically paralyzed three-year-olds in the PICU. Children were monitored continuously for 96 hours post laryngotracheoplasty with polysomnography (PSG) which involved the use of electrodes to monitor brain activity (EEG), eye movement, and muscle tone (electromyelogram [EMG]). Respiratory effort, limb movement, nasal and oral air flow, cardiac rhythm, and arterial oxygen saturation was measured through sensors. Two general distinctive characteristics of sleep are seen on EEG, rapid eye movement (REM) and non-rapid eye movement (NREM) sleep. NREM sleep can further be broken down into four different stages ranging from 1 (the most easily to be aroused from) to 4 (the hardest to be aroused from); stages 3 and 4 are known as slow wave sleep (SWS). A marked decrease in EMG activity and specific eye movements is seen in REM sleep. Neuromuscular blockade inhibits EMG activity and eye movements making it impossible to distinguish between REM sleep and wakefulness 
during neuromuscular blockade. For study purposes, non-NREM sleep was used characterizing PSG data not meeting NREM sleep stage criteria; the child may have been awake or in REM sleep.

Continuous data were collected in 12-hour periods beginning at 7 p.m. and ending after a total of 96 hours of data collection. Data included administered medications (e.g. neuromuscular blockade, sedatives) and endotracheal suctioning time recorded hourly from the nurses' notes. Other variables included movement; increases in heart rate, blood pressure, pupil size, and/or tearing beginning two hours following PICU arrival. No pain or sedation scales were used. Both children were female, approximately $14.5 \mathrm{~kg}$, one Caucasian and the other African-American (Carno et al., 2004).

The first 24 hours of recordings for the first patient were lost due to technical recording error resulting in 72 hours of analyzable data for the first child and 96 hours for the second. As expected, the EMG remained unchanged and no extraocular movement was recorded throughout data collection so PSG could not be used to determine REM sleep. Differences were recorded between time periods (day 1 to day 4 ) and between subjects in regards to proportion of time spent in various stages of NREM sleep. The first child did not demonstrate Stage 3 sleep and neither child progressed to Stage 4 sleep. Differences in total sleep time by stage were found between the two children: Stage 1 (50\%, 11\%); Stage $2(41 \%, 63 \%)$; non-NREM ( $9 \%, 22 \%)$ for child 1 and 2 respectively. Stage 3 sleep was reported in the only the second child (4\%). Children in the study demonstrated increased time in lighter sleep stages (Stages 1 and 2) at the expense of the deeper sleep stages ( 3 and 4 ) than normal 3-year-old children. Medications (sedatives, paralytics, and benzodiazepines) and endotracheal suctioning was not significant in 
changing sleep states. A greater proportion of sleep occurred during the day in the PICU child with considerable sleep fragmentation noted at all hours. Limitations of this pilot study included variability in medications used for sedation and neuromuscular blockade, small sample, and a loss of 24 hours of data in one subject due to recording errors (Carno et al., 2004).

Al-Samsam and Cullen (2005) conducted a study of 11 children between the ages of 3 and 21 months in a United Kingdom PICU who were intubated and mechanically ventilated to measure the quantity and quality of sleep captured through EEG and the effect of environmental factors on sleep factors. All children were sedated with continuous infusions of morphine and midazolam and chloral hydrate and antihistamines as needed. Children receiving muscle relaxants or Propofol were excluded. Measures included the Pediatric Index of Mortality (PIM) scores for severity of illness by Shann et al. (1997), noise levels using a noise dosimeter at head of bed, and staff interventions, defined as any direct tactile stimulation, were categorized into three levels based on intrusiveness: mildly intrusive (e.g. flushing intravenous catheters, feeding, and comfort measures), moderately intrusive (daily activities such as vital signs, diaper changes, examinations), and severely intrusive (e.g. suctioning, physiotherapy, and radiography). Sleep was monitored for 24 hours divided into 12-hour increments of days and nights measuring PSG (EEG, EMG, and electrooculogram) capturing 30-second epochs. .

Each epoch was manually scored on quiet time (designated EEG pattern, absence of body and eye movements), active sleep (REMs, designated EEG pattern), wake (eye open or opening and closing), or indeterminate stage (all periods not fulfilling criteria for other stages). Total sleep time (TST) was defined as the total time, in minutes, of quiet 
sleep, active sleep, and indeterminate stage during the total duration of the study period. Sleep efficiency, expressed as a percentage, was defined as TST divided by total study period (Al-Samsam \& Cullen, 2005).

Clinical diagnosis, surgical interventions, and medications were obtained from medical records. All the children were intubated, mechanically ventilated, and sedated with midazolam and morphine infusions. The majority of the children $(60 \%)$ were postoperative cardiac surgery. Sound level analysis could only be completed in 10/11 patients due to technical difficulties. Small statistically significant differences were between for daytime values $(M=73, S D=2 \mathrm{dBA})$ than nighttime $(M=69, S D=3 \mathrm{dBA})$ with both the highest day and night levels recorded at $103 \mathrm{dBA}$. Minimal 24 hours levels were recorded at night ( $48 \mathrm{dBA}$ ). Levels of $\geq 75 \mathrm{dBA}$ were recorded between 14 and $24 \%$ of night time hours. Wake states were recorded in children in $57 \%( \pm 15 \%)$ of episodes in which the maximum noise level was $\geq 75 \mathrm{dBA}$ for three or more consecutive minutes. A mean duration of 240 minutes $(S D=90)$ was recorded for staff contact with the child in a 24-hour period. Means for amount of noncontiguous interventions for mild, moderate, and severe interventions were recorded as $8(S D=4), 21(S D=7)$, and $10(S D$ $=3)$, respectively with the severe category having a statistically longer duration $(M=15$, $S D=5$ minutes) than the mild and moderate ones $(M=4, S D=2$ minutes each). During the entire 24-hour study period, the longest intervention-free time was reported as $99 \pm$ 38 minutes (Al-Samsam \& Cullen, 2005).

Active sleep was significantly reduced in the 24-hour period $(M=3 \%, S D=4 \%)$ and the longest sustained sleep period was reduced for age to a mean of 194 minutes ( $S D$ =79). There were no statistically significant differences in the percentages of TST, 
wake, quiet sleep, and active sleep stages between daytime and nighttime of total study period with high night wake episodes $(M=40, S D=20)$. The mean nighttime TST was between $106-688$ minutes $(M=569, S D=166)$ which falls within reported norms for healthy children of similar age (540 - 600 minutes). REM sleep was severely reduced to a mean of $3 \%$ of total sleep time, considerably less than the $30 \%$ of total sleep time achieved by normal, healthy, similarly-aged children. Four of the patients were receiving the neurotransmitter Dopamine, a powerful REM sleep suppressant. Not controlling for this variable, as well as other drugs such as antihistamines and chloral hydrate, presents a study limitation (Al-Samsam \& Cullen, 2005).

Patients in the ICU setting are the most vulnerable to stress. Lusk and Lash (2005) identified the three main categories of stressors in adult ICUs patient as psychological (danger of death, social isolation), treatment-related (tubes, painful procedures, artificial ventilation), and environmental (unfamiliar surroundings, sights and sensations, activity and noise, sleep deprivation). Research indicates environmental stressors frequently exceed regulatory recommendations with patient overstimulation from noise, lights, and sleep deprivation resulting in adverse outcomes; many of these stressors interacting with each other. Few studies have been conducted relating to PICU stressors resulting in the need to extrapolate outcomes from NICU and adult studies. Research found related to the combined effects of light and sound; minimal, in NICU, were found relating to light only.

Gaps noted were effects of excessive noise levels investigating new respiratory modalities (e.g. high-frequency oscillators, high-frequency jet ventilators, and extracorporeal membrane oxygenation. Study weakness included lack of consistency 
with scales for measuring noise (e.g. Hz, dBA, dBC) and light levels creating confusion interpreting results. Duration of measurement was not clear in some studies and variability in the location of measuring devices may have affected measurements. It was not clear how appropriate sound and noise levels were determined by recommending agencies. Additionally, guidelines prior to 2000 were not found indicating a lack of revisions made based on significant hospital environment changes influencing noise and light levels.

Studies reflecting sleep disruption in children are lacking. A large study weakness included loss of data from equipment malfunction; the lack of studies makes any loss of data particularly significant. Effects of other equipment (e.g. electric beds, air loss mattresses) which may interfere with sleep-wake cycles was not measured. Many medications, such as neurotransmitters, affect sleep; controlling for medications would make study results more powerful. Many of these studies lacked a comparison group; although this may present an ethical issue in this vulnerable population.

Fears

The child's fears are an important component to their hospitalization response. Hospitalized children report a multitude of fears relating to their experience. Research indicates the most common fears are related to pain and nursing procedures (Carney et al., 2003; Lindke et al., 2006). Preschool aged children experience common developmental fears such as fear of darkness, separation from parents, and being exposed to unfamiliar people (Salmela et al., 2009). Older hospitalized children have reported fears related to nursing procedures (Koening et al., 2003; Salmela et al., 2009), strange environments, equipment, pain (Wennström et al., 2008; Salmela et al., 2009), lack of 
privacy and discretion, restricted free will, rejection, bodily injury (Wennström \& Bergh, 2008), injections and needles (Salmela et al., 2009). Unrealistic fears, lack of information, surgery, and disease symptoms have also been identified as fear provoking (Wennström \& Bergh, 2008; Salmela et al., 2009).

\section{Procedures.}

Highly invasive procedures are a necessary component of care for children hospitalized in a PICU. Procedures children endure may include intubation, ventilation, suctioning, invasive intravenous or arterial lines, chest tubes, continuous infusions of fluids and/or medicines, urinary catheters, venipunctures, bronchoscopy, dressing changes, bone marrow aspirations, lumbar punctures, and turning. Children may have to undergo procedures in other areas outside of the PICU (e.g. radiology, surgery). Serious adverse complications, pain, discomfort, and increased mortality are associated with these procedures (Morrow, 2008). Many of these procedures are not only a tangible reminder of the severity of the child's condition but also affect the child's appearance resulting in a very intimidating, frightful effect for the children and their families (Haines et al., 1995).

Rennick, Johnston, Dougherty, Platt, and Ritchie (2002) used a prospective cohort design in two Canadian hospitals to compare 60 PICU children to $60 \mathrm{GCU}$ children on psychological outcomes and contributing factors. As each PICU child was admitted to the study, they were matched on age (+/- one year) and illness category with a GCU child. Illnesses were categorized according to planned or unplanned admission, acute or chronic illness, and surgical intervention required. Data were collected at three time 
points (just before PICU or GCU discharge, 6 weeks post-hospital discharge, and 6 months post-discharge) on children 6-17 years old and their parents.

Instruments used included the Pediatric Risk of Mortality Scale (PRISM) to measure severity of illness, Invasive Procedure Score (IPS) modified from the therapeutic intervention scoring system to record number and types of invasive procedures, length of stay (LOS), Post Hospital Behavior Questionnaire (PHBQ) comparing pre- and posthospital behavior, Children's Impact of Event Scale (CIES) modified by the researchers from the Impact of Events Scale (IES) to measure children's responses to traumatic life events, Children's Health Locus of Control Scale (CHLOC), and the Child Medical Fear Scale (CMFS). Three child questionnaires (CIES, CHLOC, CMFS) and one parent questionnaire (PBQI) were mailed to the families 6 weeks and 6 months following hospital discharge. Parents were asked to read questions to the children 8 years of age and younger. Follow-up telephone calls were made subsequent to the mailings (Rennick et al., 2002).

No significant group differences (PICU vs. GCU) were found for child's age, previous hospitalizations, mother's education, sex, language, and expected or unplanned admission. Significant group differences were found for LOS (PICU: $M=13, S D=8.5$ days; GCU: $M=8.8, S D=6$ days), invasive procedure scores (PICU: $M=88.8, S D=$ 71.1; GCU: $M=22.5, S D=20$ ), and severity of illness scores (PICU: $M=25.3, S D=$ 22.8; GCU: $M=4, S D=11$ ). Not surprisingly, children in the PICU received significantly more sedation and analgesics than the GCU group. Findings indicated children were exposed to an average of 89 tissue damaging and non-tissue damaging invasive procedures during their PICU stay compared to an average of 23 invasive 
procedures for children on GCUs. Children in the PICU experienced more hospital readmissions by 6 weeks following discharge than the GCU group. No group differences were found at 6 weeks and 6 months for children's psychological responses after PICU and GCU hospitalization. Children's age and illness severity were significant predictors of their CHLOC scores 6 weeks after hospital discharge with those younger (7-11 years) and more severely ill having a lower sense of control over their health. Higher maternal education was a significant predictor of higher CHLOC scores. Six months after discharge, the child's age remained the only significant predictor of CHLOC scores with younger children having a lower sense of control over their health (Rennick et al., 2002). Children subjected to higher numbers of invasive procedures reported higher CIES 6 weeks post-hospital discharge indicating more avoidance behaviors and intrusive thoughts. Absence of a family member visiting was a significant predictor of higher CIES scores 6 months after hospital discharge. Problematic responses to trauma measured on the CIES indicated 25\% of the children had high mean scores at both time points post-hospital discharge indicating relatively high negative responses that did not resolve over the 6-month study period (Rennick et al., 2002).

At 6 weeks and 6 months post-hospital discharge, main and interaction effects of the child's age and number of invasive procedures were significant predictors of CMFS scores with younger children exposed to higher medical fears. Confounding variables included previous numbers of hospitalizations, child's sex, and family visiting with only females found to have higher fear scores. Statistically significant CMFS scores were reported in $17.5 \%$ of the children 6 weeks post-discharge and $14 \%$ six months postdischarge (Rennick et al., 2002). 
As long as six months post-discharge, children reported a lower sense of control over their health, more medical fears, and more symptoms of posttraumatic stress (PTS), especially for younger, more severely ill children who had experienced a higher number of invasive procedures regardless of hospitalization location. Overall, findings suggest that invasiveness coupled with LOS and illness severity have adverse long-term effects, especially for young children (Rennick et al., 2002).

Study limitations included the broad age range used for study inclusion necessitating the use of outcome measures for children as young as 6 years. The PBQ may have been inappropriate for children older than 12 years. Additionally, the instrument was not intended to assess long term behavioral changes therefore may not have been a sensitive indicator of behavioral changes longer than 2 weeks post-discharge because of the recall aspect of the measure. Additionally, the CIES was modified from the adult scale. Child reports items on the intrusion and avoidance behaviors on the scales did not perform in the factor analysis as expected. A number of possible limitations related to unsupervised home questionnaire completion include parents influencing responses when reading to children, providing "good" responses due to social desirability, and children's answers influenced by friends and family. Lastly, the PRISM was developed to be used to assess PICU patient mortality; this study included its use in measure of illness severity in GCU children as well (Rennick et al., 2002).

A secondary analysis was conducted to identify PICU patients at highest risk for developing persistent psychological sequelae post-hospital discharge (Rennick et al., 2004). This sample of 60 children from the PICU children aged 6 to 17 years was divided into a high-risk group and a low-risk group based on the children's illness 
severity and invasive procedure scores. A post-hoc decision was made to use cutoff scores at the beginning of the upper quartiles in defining the high-risk group (PRISM $\geq$ 34 , IPS $\geq 21, n=20)$. All other children comprised the low-risk group $(n=40)$. Six weeks post-discharge, children in the high risk group had significantly more medical fears, lower sense of control over their health, and more symptoms of PTS than the lowrisk group with invasive procedures identified as the most important factor for differences. At six months post-discharge, significantly higher prevalence of PTS was found in the high risk group. Group differences in children's medical fears or health locus of control scores at six months were not significant. Invasive procedures and severity of illness had no effect on children's medical fears or health locus of control scores at six months. Severity of illness was found to be the strongest predicator of PTS (Rennick et al., 2002).

Study limitations were created by instruments used with psychometric testing conducted in different age groups or time frames than used in this study. Many of the items in the PHBQ are relevant to behaviors in younger children (e.g. the need for a pacifier); the results in older children may be in part related to lack of questions relating to behavior and distress in that age group. Additionally, the instrument was tested and primarily used in post-operative children following hospitalization and may not be a sensitive measure of changes further than 2-weeks post-hospital discharge. The CIES was modified from an adult scale for this study; however, intrusion and avoidance subscales could not be used resulting in a total score calculated as an overall indicator of stress. Children's intrusion and avoidance behaviors are more than likely not similar to adult populations creating challenges when attempting to assess with an adult scale. 
Potential limitations may also be related to use of the PRISM scale in measuring illness severity in children in the GCU; this instrument was developed as a measure of illness severity for children in the PIC in the initial 24 hours. Additionally, the lack of collecting post-discharge data in a controlled manner created a potential bias limitation.

Questionnaires were mailed to the families following discharge with parents asked to read them to children younger than 8 years; families may have influenced children's responses (Rennick et al., 2002).

A later multisite study by Rennick et al. (2011) tested the psychometric properties of the Children's Critical Illness Impact Scale [CCIIS] (Rennick et al., 2008), a measure of psychological distress for young children. For purposes of comparing contrasting groups, children $(n=84)$ between the ages of 6 and 12 years from one of four Canadian PICU and children $(\mathrm{n}=88)$ from an ear-nose-and-throat $(\mathrm{ENT})$ same day surgery clinic were enrolled in the study. Parents and children in the PICU group were recruited following PICU discharge. Two months after hospital discharge, a parent explanation letter, the CCIIS, the CMFS, and the Revised Children's Manifest Anxiety Scale were mailed to the home. Study reminders were made two weeks following the mailing through telephone calls to the parents with a second thank-you/reminder call two weeks later. Two weeks after return of questionnaires, the CCIIS was mailed out a second time in order to conduct test-retest reliability testing. ENT group participants were recruited 6 to 8 weeks following day surgery by telephone or during a follow-up clinic visit. Following consenting, the CCIIS and parent explanation letter were mailed to the home. Reminder calls were conducted as in the PICU group. 
Demographic and medical information was collected from the medical record. An IPS was calculated as in indicator of illness severity reflecting the total number of tissuedamaging and non-tissue damaging invasive procedures children received during their PICU stay. IPS was calculated during the postoperative recovery period for children in the ENT group. Items were generated following a literature review and thematic analysis of qualitative data from focus groups with 52 children between 6- and 12-years-of-age and their parents. Factor analysis supported these subscales: (1) worries about getting sick again, (2) feeling things have changed, and (3) feeling anxious and fearful about hospitalization (Rennick et al., 2011).

Children in the PICU group were slightly older than those in the ENT group $(M=$ 9.4, $S D=2.1$ years; $M=8, S D=1.9$ years respectively). The mean length of PICU stay was 3.7 days $(S D=3.8$ days). As expected, IPS were much higher in the PICU group ( $M$ $=57.7, S D=90.9)$ than the ENT group $(M=2.5, S D=0.65)$. More than $60 \%$ of children in both groups received narcotic analgesics. More children in the PICU group received non-narcotic analgesics (68\%), sedatives (44\%), paralyzing agents (14\%), and anesthetics following surgery (23\%) than the ENT group (14\%, $1 \%, 1 \%$, and 0 respectively). Most children in both groups (94\%) remembered being in the hospital; $75 \%$ of those admitted to PICU remembered being there (Rennick et al., 2011).

Children in the PICU group reported higher CCIIS mean scores $(M=45, \mathrm{SD}=$ 10.7) than those in the ENT group $(M=41, \mathrm{SD}=8.1)$; higher scores were reported more in boys than girls and in children 9- to 12 years-of-age in both groups. Test-retest reliability was examined at two-weeks finding an acceptable total ICC of 0.70 . CCIS total scores and scores with the three-factor solution were positively correlated with child 
anxiety and medical fears scores. Concurrent validity found low correlations $(r=.18, .30$ and .28) for the three factors (Rennick et al., 2011). Testing the CCIIS with a larger population could increase the power possibly resulting in stronger psychometrics.

\section{Memories}

Anesthetics, analgesics, and sedatives have been found to disrupt the memory process. Propofol causes total amnesia for the duration of administration in children and is associated with retrograde amnesia for events prior to administration in adults and children (Miner et al., 2005; Quraishi, Girdharry, Xu, \& Orkin, 2007). Benzodiazepines also have an amnesic effect in children (Kain et al., 2000). Consistent with adult findings, evidence suggests that implicit learning takes place during sedation although explicit recall in children is severely impaired (Pringle, Dahlquist, \& Eskenazi, 2003; Stewart, Buffet-Jerrott, Finley, Wright, \& Valois Gomez, 2006). This sensory-based processing occurs but conceptual recall may be poor with sedation in children (Dow et al., 2012). Sleep alterations in PICU may also impact recall as children's explicit, or declarative, memory is improved by sleep, especially for emotional information (Prehn-Kristensen et al., 2009; Wilhelm, Diekelmann, \& Born, 2008).

Seminal work involving children's perceptions and recall of the PICU was conducted by Barnes and colleagues $(1972,1975)$ and Vanek (1979) examining the accuracy of children's memory by comparing researcher observations and school-aged children's retrospective perceptions. Findings indicated the children exhibited a great deal of distortion in their perceptions of events, perhaps indicative of their limited capacity to verbalize what they think and remember. They found children were intensely vigilant to and extremely apprehensive about treatments and procedures. 
Barnes, Kenny, Call, and Reinhart (1972) studied psychological outcomes in 11 children between the ages of 5 and 14 years on neuro-endocrine and behavioral responses in children who were overtly anxious and those appearing to have better emotional control. The children had been admitted for correction of a variety of cardiac defects. Degree of anxiety was assessed by Co-Investigators (child psychiatrist and a pediatric nurse specialist) during hospitalization and following hospital discharge. Neuroendocrine data were collected through urinalysis of 17-hydroxycorticosteroids at the same time points. The Co-investigators defined children with extreme verbal and motor behavior, in either direction, which interfered with the ability to cope with impending surgery or treatment, as severely anxious. Children showing fear or concern but still able to socialize were considered mildly anxious. Seven children were identified as "severely anxious" and four as "mildly anxious". Results found 8 of the 11 children were not accepting of their surgery; seven in the severely anxious group. Children in both groups had pre-operative teaching, intensity varying with their level of anxiety; both groups were found to be underprepared. Three of the 11 children expressed death fears. Two of the three had had experienced the death of a close relative in the two years preceding their surgery. Parents and children in the severely anxious group had physical symptoms of illness prior to surgery. Measures of urinary steroids for both groups showed increased levels before surgery, with suture removal, and on outpatient visits, but no significant differences between groups.

A later observational study by Barnes (1975) investigated the perceptions of PICU experiences, recall, and perceptual distortions in 13 children between 6 and 13 years after cardiac surgery. All but one of the children came from 2-parent families. The 
average time interval from diagnosis to surgery was 6.5 years. Only one of the children had had previous surgery for palliative correction. All of the children had been previously hospitalized, primarily for cardiac catheterization. The average hospitalization stay was 12 days with an average PICU stay of 2.5 days. Children described negative PICU reactions such as fatigue, sadness, pain, anger, boredom, fear, and confusion and displayed high levels of awareness and alertness. They had detailed recall of the environment, experiences with themselves and other children, and dream experiences in the PICU. In addition, Barnes (1975) found that procedures and treatments evoked the most distortion. Some children tried to mouth words to communicate while intubated, described procedures performed on them, and expressed confusion about the suctioning procedure and the location of the endotracheal tube. Contrary to adult ICU findings, children did not fear death, possibly explained by the age differences, and were more focused on pain relief. Children were possibly focused on pain relief due to under medication for pain.

Playfor, Thomas, and Choonara (2000) conducted interviews with two groups of United Kingdom children between 4 and 16 years of age following PICU discharge. Group A included 24 children over 4 years old admitted to PICU during 12 consecutive weeks and Group B investigated 14 similar mechanically ventilated PICU children in the seven months prior to the onset of the study. Group A were recruited during their PICU stay with interviews occurring in the GCU. Parents of all younger children were present and older children were given the option of having parents present. Following the interview, children were asked to draw a picture of what it was like to be in the PICU. Group B were contacted by telephone with interviews conducted at a mutually 
convenient time. The unit's standard of care includes a standard sedation regime of continuous intravenous infusions of midazolam and morphine with additional oral sedation using chloral hydrate and antihistamines as required in mechanically ventilated children. Following achieving adequate sedation, a continuous infusion of a neuromuscular blockade may be added. .

The median duration of PICU stay was three days (range $1-11$ days), 25 children $(66 \%)$ were mechanically ventilated and seven $(18 \%)$ received neuromuscular blocking agents. Interviews with Group A occurred 1-12 days following PICU discharge and Group B interviews ranged between 4-28 weeks following discharge with no differences found between group responses. PICU stay ranged between 1 and 11 days (Median $=3$ days). More than half the children $(n=25,66 \%)$ remembered the PICU. Clinical diagnosis is described; however, diagnoses per group are not clear. Children identified 44 specific recollections of which $60 \%$ were neutral, $25 \%$ positive, and $15 \%$ negative. Children's bad memories included being unable to speak because of the endotracheal tube, lack of sleep, not knowing the time of day, and being hungry. Children also addressed remembering pain (29\%), being scared (21\%), and being unable to sleep (16\%) because of noise or discomfort. Twenty five children had been mechanically ventilated and received midazolam and morphine. Twelve children (48\%) were able to recall the PICU and four remembered some parts of mechanical ventilation. Seven children had been treated with neuromuscular blocking agents and none remembered their pharmacological paralysis. Mechanically ventilated children receiving midazolam and morphine remembered little of their intubation. Midazolam causes retrograde amnesia which may account for decreased recollections. Two children experienced unusual 
dreams following PICU discharge: one dreamt about invasive procedures and the other was only able to describe in terms of it being frightening. Nine children drew pictures which primarily depicted themselves lying in a bed or standing in the hospital (Playfor et al., 2000).

Study limitations included the small sample and use of prospective and retrospective sample recruitment in two different groups to compare outcomes. Large data collection time differences were apparent between the groups with possible variable variations such as PICU policy and procedures as well as the possibility of staff contamination resulting from knowledge of the study. A single investigator conducted both interviews however it is unclear if it is the same one (Playfor et al., 2000).

Similar findings were reported by Karande, Kelkar, and Kulkarni (2005). A convenience sample of 50 children from India between 5 and 12 years old with a variety of complex, medical diagnoses described their critical care experiences during interviews 1 to 5 days following their PICU transfer to the GCU. Twenty-two open and closedended questions guided the interview. Following the interview, the children were asked to draw pictures depicting their PICU stay. Children who received mechanical ventilation were excluded due to their death or severe neurological sequelae.

The majority of the children (62\%) were socioeconomically disadvantaged. The PICU length of stay was between 1 and 29 days (Median $=5$ days). All children remembered the PICU and 36 identified severity of illness as reason for PICU admission. Thirty seven (74\%) of the children had neutral recollections, nine (18\%) negative, and eight (16\%) had positive recollections of their PICU stay. Recollections included: feeling scared $(n=32,64 \%)$, especially of needles; being in pain $(n=37,74 \%)$; thirsty $(n$ 
$=7,14 \%)$; hungry $(n=8,16 \%)$; and sleep disturbances $(n=9,18 \%)$. Children also expressed memories of nasogastric tube insertion, chest tubes, lumbar punctures, restraints, and dead children with $26 \%$ reporting seeing dead bodies and families mourning. Vivid recall of PICU events was described by $96 \%$ of the children possibly resulting from inadequate sedation and analgesia management (Karande et al., 2005). A major limitation to this study was the exclusion of intubated children. This population represents the most critically ill who receive the highest number of invasive procedures, sedation, and analgesia. Although the researchers wanted to allow the children to settle in to the GCU environment, variation in time in GCU may have affected recall creating a bias. Additionally, researchers were employed in the PICU possibly affecting participant response, particularly if the care for any of the children in the study (Karande et al., 2005).

A cross-sectional study was conducted by Board (2005) examining effects of PICU hospitalization upon transfer to GCU using a convenience sample in 21children between 7 and 12 years old ( $m=9.6$ years) recruited from a children's hospital in the east coast or Midwest America. None of the children had been previously hospitalized, $38 \%$ had an unexpected admission, $57 \%$ were white, and $43 \%$ were medical admissions. Structured interviews were conducted within 24 hours of transfer to a GCU. Parents were allowed to stay with instructions given to not talk during interview. PICU recollections were investigated using researcher developed, open-ended questions; anxiety with the Child Drawing Hospital (CDH); and coping strategies measured by the Schoolagers Coping Strategies Inventory (SCSI); which was read to the children. The $\mathrm{CDH}$ scoring is divided into three sections and a total score. Part A measures anxiety and 
contains 14 items with anchors of 1 for lowest anxiety level to 10 for highest; Part B measures pathological indices; and Part $\mathrm{C}$ is the Gestalt rating of the drawing on a scale of $1-10$. Original alpha coefficient for reliability was .79 The SCSI is a 26-item, self report instrument containing two scales: frequency and effectiveness. Original internal consistency was reported as .75 .

Ten overarching themes were identified for children's PICU recollections: what was remembered overall, what was good, what was bad, people, equipment, emotions experienced, symptoms experienced, invasive procedures/equipment, who or what helped, and visitors remembered. Most children (67\%) described vague memories of the PICU experience which included feeling badly (scared, hurting, tired), procedures, and equipment. Memory of invasive or intrusive procedures was reported by only $33 \%$ of the children. One of seven children had memories of intubation. Frequency and effectiveness of coping was low in these children compared to children with asthma, recurrent abdominal pain, and normal, healthy children reported in other studies (RyanWenger, 1996). Sleeping or taking a nap was reported by all the children as the most frequently used coping strategy. Other strategies included talking to someone and praying; although, the latter was infrequently used. Three statistically significant relationships were found when investigating relationships between demographics, hospital experience, experience recollections, and emotional status. There were significant, negative correlations between intubation length and the SCSI frequency and effectiveness scales indicating children with longer intubations used less coping strategies and those used were ineffective. Additionally, children with no PICU recollections had a negative correlation with the $\mathrm{CD}$ : $\mathrm{H}$ total score indicating children who did not initially 
remember much about their stay were more anxious than those with immediate memory. Ten children had scores indicating average levels of anxiety, two above average anxiety, six had low levels of anxiety, and three did not complete the instrument. Higher anxiety scores were identified in children not remembering their PICU stay (Board, 2005). No children mentioned the cardiac monitors, ventilators, or noises from other machines which has been found to be a source of stress for PICU parents (Board \& Ryan-Wenger, 2003).

Common to PICU stays, children's memories may have been affected by administration of opioids and benzodiazepines. Study weaknesses included a small sample and lack of data collection relating to number of PICU invasive procedures. Only seven of the children were intubated with most having a peripheral IV reflecting lower acuity. Data were not collected on other common PICU procedures such as nasogastric or orogastric tube placement, suctioning, arterial or central line placement, lumbar punctures, and insertion of urinary catheters (Board, 2005).

In summary, multiple factors are implicated in alteration of children's memory which include cognitive level, resource availability, medication use, and sleep deprivation. The majority of the research studies found children able to recall many aspects of their PICU stay, finding it highly anxiety-provoking. As with adult ICU research, the majority of children accurately recalled components of their PICU stay. The PICU environment and requisite care increases the probability that children will experience delusional memories placing them at risk for negative outcomes.

Children experience adverse psychological responses following PICU admission that persist far past their hospital discharge. These children may manifest long-term 
emotional dysfunction, negative physical health outcomes, and poorer health-related quality of life (Dow et al., 2012). In addition to high levels of knowledge and impeccable critical care skills, environmental factors and cultural differences also affect the child's recovery. Findings on the psychologic impact of children's PICU hospitalization depict both resilience and distress with adverse sequelae long past discharge (Colville, 2008).

Rennick and colleagues $(2002,2004)$ were the only researchers found to use longitudinal methodology with comparison groups to investigate children's fears and memories. This created a major weakness in the studies as the ability to detect changes between PICU and GCU groups as well as those occurring over time could not be examined. Additionally, SES, race/ethnicity, and single or two-parent families were not clearly identified in all the studies making generalizability difficult to other demographics. Many of the studies relied solely on interview technique limiting the ability of the younger child to clearly depict their thoughts. Children's' responses may have been affected by parental presence during the interviews. Collecting data on children with a wide range of diagnoses or unclear diagnoses was a weakness in many of the studies creating the inability to describe any relationship between diagnosis and memories.

\section{Child Responses to PICU}

Children in PICU are more critically ill, subjected to more invasive procedures, and are more likely to receive sedation and analgesia than GCU children (Rennick et al., 2002; Rennick et al., 2011). The highly invasive technological interventions, severity of 
child's illness, high levels of parental distress, and lack of control over events have been identified as significant factors for the child's development of negative physical, emotional, and developmental sequelae as a result of PICU hospitalization (Rees et al., 2004; Rennick et al., 2002; Rennick et al., 2004).

The child's physical, emotional, social, and cognitive development helps to shape the perception of the stressful hospital situation (Aley, 2002). Factors negatively affecting a child's coping response to hospitalization include younger age, male gender, and family factors such as increased numbers of family stressors, lower socioeconomic status, and highly anxious and less educated mothers (Small \& Melnyk, 2006). Melnyk (2000) found that children's emotional and psychological predisposition, coping skills, and prior hospital experiences affect the child's responses. Medical experiences and hospitalization can result in developmental delays, loss of newly acquired developmental skills, loneliness, isolation, and PTS for the child (Aley, 2002; Melnyk, 1994).

Of particular importance to the child's hospital adjustment are the parent's emotional state, quality of participation in child's care, and support of others both during and after discharge (Melnyk, 1994). Small and Melnyk (2006) found maternal state anxiety was the most significant predictor of children's later aggressive and hyperactive behavior three and six months following PICU hospitalization. Melnyk and Feinstein (2001) found high maternal anxiety leads to high anxiety in the child.

A prospective, cohort study was used by Muranjan, Birajdar, Shah, Sundaraman, and Tullu (2008) in India investigating associations of PICU hospitalization and adverse psychological outcomes, contributing factors, and symptom duration. Consecutive patients $(n=30)$ over 5 years without prior PICU admission (Cases) were compared with 
30 age and sex-matched children without previous PICU admissions from the GCU (Control). Interviews were conducted within 24 hours of discharge from PICU and controls were interviewed the day of discharge with a second interview one month later. Severity of illness was measured after 24 hours of admission using the Pediatric Risk of Mortality- III (PRISM- III). Data was collected on the Therapeutic Intervention Scoring System (TISS-76) measuring intensity of critical care interventions; the Temperament Measurement Schedule (TMS), a parental report of the child's premorbid disposition on five domains: sociability, emotionality, energy, distractibility, and rhythmicity; the IES measured intrusive symptoms (nightmares, imagery, insomnia, and emotional reactions) and avoidance behaviors (numbing and avoidance); Birleson Depression Self-Rating Scale; Rosenberg's Self-Esteem Scale, validated for children 7 - 13 years, measuring symptoms of mood, appetite, pain, sleep, ad interest with a score of greater than or equal to 13 indicative of depression. To simplify, the response format was changed from numbers to a line and all questionnaires were read to the children.

Mean age was $8.5 \pm 5.2$ years for children in PICU and $8.9 \pm 6.2$ years for children in GCU. Groups were comparable for demographics and socioeconomic status. Length of stay for GCU exceeded PICU ( $11 \pm 11.8 ; 6.6 \pm 4.9$ days $)$. Diagnoses were significantly different between the units with infectious (33\%), cardiovascular (23\%), and neurological (20\%) in the PICU group and genitourinary (27\%), hematological (20\%), and respiratory (17\%) in the GCU group. Similar findings for premorbid temperament were found in both groups in all domains except energy with higher energy seen in the GCU group than PICU group ( $n=7 ; 1$ respectively); this finding did not influence psychological outcomes. Mean intrusion scores measured on the IES were significantly 
higher in children in the PICU than those in GCU and $43 \%$ of PICU children developed intrusive thoughts compared to $6.7 \%$ of GCU children. Intrusive thoughts were strongly correlated with the number of interventions. Intrusive thoughts were images related to PICU hospitalization, bad dreams, strong feelings, thoughts, and trouble falling asleep due to PICU images or thoughts. The mean PRISM score was $3.27 \pm 3.44$. The PICU group had statistically significant higher mean TISS scores at both times (8.97 \pm 4.22 ; $5.85 \pm 2.80)$ than the GCU group $(1.4 \pm 1.6 ; 0.9 \pm 0.74)$. Initial and one month postdischarge results did not differ significantly (Muranjan et al., 2008).

Study limitations included the use of scales not previously used with children. The IES was tested for children over the age of 8 years and the depression scale for children between 7 and 13 years. Attrition rate was high given the already small sample with only 17 PICU and 22 GCU children available for follow up. It is unclear if the reported mean PRISM score reflected both units. Statistically significant results may have been found in the follow-up data with a larger sample (Muranjan et al., 2008).

Wilson et al. (2007) recruited hospitalized and non-hospitalized children to develop and test psychometric properties of the Barton Hospital Picture Test (BHPT) which consists of eight drawings of specific hospital situations: admission, separation from parents, being examined by a doctor, receiving oral medications, and injection, being in operating room, and being in the playroom. Using quota sampling, participation included 9 to 10 children at each year of age from 5 to 9 years recruited from a Midwestern children's hospital and a university health science center $(n=48)$ and rural and urban community settings $(n=45)$. The sample was predominantly white $(88 \%)$, from middle- to upper-class backgrounds, female (58\%), and approximately half of the 
hospitalized children had chronic illnesses. Interviews were conducted in the hospital room or the child's home. Parental presence was permitted with instructions to not to engage in the interview process. The researchers showed the children a BHPT picture, given a brief introduction to the picture, and asked the child to tell a story. Following the last story, children were asked their views on their hospitalization: what is the best thing about being in the hospital, the worst, and how would they make it nicer for children. Following training, pediatric nurses served as the interviewers. Five categories emerged: anxiety-fear, anxiety-defense, aggression, dependency, and total stress. The revised instrument was found to have good test-retest reliability $(\alpha=.83)$, excellent interrater reliability $(\alpha=.86)$, and appropriate construct and discriminant validity.

To investigate children's views of hospitalization through their own voices, a qualitative secondary analysis using the community children's data was conducted by Wilson, Megel, Enenbach, and Carlson (2010). Codes from the first study were removed and the five categories identified were avoided. Data were analyzed using content analysis and three themes and their relationships were defined. The primary theme identified was being alone. When they were alone they were afraid of scary things, there was uncertainty about what was going to happen to them, and they were not at home which led to feeling scared, sad, or mad. One child voiced "She's mad that her parents aren't there and that she's alone too" while another indicated being "scared because she doesn't know what they're going to do and she doesn't have her parents with her and she thinks they're going to give her a shot or something" (Wilson, Megel, Enenbach, \& Carlson, 2010, p. 98). Less extensive themes included being aware of the possibility of good or bad hospital outcomes and viewing hospitals as unique 
environments. Unlike those never hospitalized, hospitalized children used medical or hospital language with stories containing personal experiences.

Major study limitation included study participants who were primarily White, English speaking, and middle class. This greatly limits generalizability of study findings to other racial/ethnic and lower SES groups. Comparing findings to other cultures is challenging as this study was conducted in Midwestern America (Wilson et al., 2010).

In their study of 36 children 6 to 18 years $(M=12.6$ years), Levy, Kronenberger, and Carter (2008) examined children in chronic life-threatening $(n=36)$, acute lifethreatening $(n=9)$, chronic non-life-threatening $(n=20)$, and acute non-life-threatening $(n=20)$ categories to investigate the relationship between chronicity and severity of illness and child adjustment in and out of the hospital. Potential participants were identified from admission lists of inpatients referred to a consultation-liaison service from another study with families invited to participate during the child's hospitalization. Participants had a range of chronic conditions which were primarily cancer $(n=17)$, asthma (11), traumatic injuries (14), diabetes (9), and renal disorders (6). Illnesses were coded based on consultation-liaison team consensus: chronic versus acute (chronicity factor) and non-life-threatening (severity factor) with interrater reliability measured at $93 \%$.

Parents completed the Child Behavior Checklist (CBCL) to measure externalizing (aggressive and delinquent subscales) and internalizing (withdrawn, anxious/depressed subscales and somatic complaints) behavior over the previous six months. Primary nurses completed four of the seven subscales of the Pediatric Inpatient Behavior Scale (PIBS) measuring child behaviors during hospitalization: Oppositional/Noncompliant, 
Withdrawn, Distress, and Anxiety. Statistically significant results were reported for PIBS Internalizing and chronicity and severity interaction effects; children with acute illnesses reported higher in-hospital internalizing behaviors than chronic illness scoring more than twice the rate of children with chronic illness on Internalizing subscales (20.7\% vs. $8.9 \%)$. Children with life-threatening illness (15.6\%) reported higher inhospital internalizing problems than those with non-life-threatening illnesses $(10 \%)$. Significant results were not reported for PIBS Externalizing and chronicity and severity interaction effects. These findings suggest children with acute or life-threatening illnesses demonstrated more behavioral problems while in the hospital. Children with chronic illnesses had lower in-hospital problems but greater behavior problems out of the hospital perhaps because their more frequent hospital stays result in higher comfort levels in the familiar hospital environment (Levy, Kronenberger, \& Carter, 2008).

\section{Factual and Delusional Memory}

Colville and colleagues have conducted extensive research on delusional and factual memory of children admitted to PICU (Colville, 2008; Colville et al., 2008; Colville \& Pierce, 2005; Colville et al., 2006). Their findings indicated an association between delusional memories and subsequent post-traumatic stress.

Colville, Kerry, and Pierce (2008) examined the extent of delusional and factual memories after PICU discharge, relationships between those memories and demographic and medical variables, and relationships between either type of memory and subsequent development of PTS in 102 children. Families of children older than 7 years were recruited over 18 months from an inner city United Kingdom (UK) PICU. Children were interviewed by an experienced clinical psychologist in the home or hospital as chosen by 
the family. A checklist using the ICU Memory Tool which was developed and tested in adult patients, facilitated recall for factual and delusional memories as well as memories of feelings. Children also completed the 8-item Children's Revised Impact of Event Scale (CRIES) measuring post-traumatic stress symptoms. This tool was developed for children between 7 and 18 years and has established reliability and validity. Scores above 17 have been found to correctly identify more than $80 \%$ of children with a PTSD diagnosis. Severity of illness was measured on the PIM and social deprivation/isolation on the Townsend Deprivation Index, derived from the UK.

Children participating were between 7 and 17 years admitted for a wide variety of medical/surgical diagnoses. More than half were white (57\%) and male (58\%). PICU length of stay was between $0-38$ days with $91 \%$ of the children requiring mechanical ventilation. Medications children received included benzodiazepines (84\%), opiates (92\%), Thiopentone (4\%), Propofol (2\%), and paralytics (43\%). Factual memory of the PICU admission was reported by 64 (63\%) of the children with 29 describing a single, fragmented memory and 35 reporting two or more PICU memories. Thirty-eight children remembered nothing about the admission despite being supplied with the ICU Memory Tool. At least one delusional memory was reported by 33 (32\%) children. These memories were described as highly disturbing and originating in the PICU in all but two cases. The hallucinations were primarily visual $(n=16)$ but children reported auditory, visual, and tactile hallucinations as well. Two children reported feeling that their parents had been replaced by imposters. Multiple hallucinations were described by 11 children beginning near the end of their PICU stay and continuing for several days after hospital discharge frequently interfering with sleep. Consistent with adult findings, a significant 
correlation was found between length of time on morphine and prevalence of hallucination (Colville et al., 2008).

Consistent with the previous study, PTS scores at three months following hospital discharge were significantly associated with emergency admission and presence of delusional memories. Report of delusional memory was significantly associated with longer length of stay and greater duration of opiates/benzodiazepines with children being nearly five times more likely to report a delusional memory if they had received opiates and/or other narcotics for more than two days. Study results found that nearly one in three children reported delusional memories associated with their PICU stay with delusional memories positively associated with higher rates of PTS. Limitations to the study include the lack of a control group and lack of assessment of withdrawal symptoms or delirium. The lack of psychometrically sound child measures necessitating using or modifying adult measures created the largest weakness (Colville et al., 2008).

Rees, Gledhill, Garralda, and Nadel (2003) conducted a retrospective cohort study to determine if PICU $(N=35)$ admission was associated with greater psychiatric morbidity in children and their parents than those admitted to a GCU $(N=33)$ from a London hospital. Two researchers reviewed logs to identify GCU and PICU children between 5 and 18 years matching them for age, hospitalization dates, diagnoses, and sex. Recruitment, interviews, and data were obtained 6 to 12 months after hospital discharge. Children and parents were initially seen together then older children were interviewed alone while the parents completed questionnaires; parent(s) were present for interviews and data collection with younger children. Discharge medical diagnoses were obtained from medical records. 
Measures included children's and parent's quantifiable perception of illness developed for this study. Illness severity and degree to which the child or parent feared for the child's life were measured using a 10-point scale; 1 (not at all) to 10 (extremely ill or very much so). The Clinician-Administered PTSD Scale for Children (CAPSC) and a semi-structured interview measured the child's psychiatric status. Children were asked to complete the IES, Birleson Depression Scale, Revised Children's Manifest Anxiety Scale, Child Somatization Inventory (CSI) measuring 35 physical symptoms present in the prior two weeks on 5-point intensity, and the Strengths and Difficulties Questionnaire (SDQ) measuring conduct, hyperactivity emotional, peer relationship problems, and prosocial behavior. The SDQ was self-reported in children older than 11 years and completed by parents about their child in those less than 11 years. Parent's self-reports included the IES, the General Health Questionnaire, The Beck Depression Inventory, and the Hospital Anxiety and Depression Scale (Rees et al., 2003).

More than half the children in both groups were male (PICU: 66\%, GCU: 55\%). Statistical differences were reported in hospital LOS (PICU: 8 days, GCU: 3 days). Higher rates were reported by children and parents for severity of illness and degree of fear of life than GCU. Parents' perceptions of illness were greater than their children for severity of illness and life threat. After discharge, four of the 35 PICU-admitted children and none of the GCU group met diagnostic PTSD criteria in both home and school setting. Additionally, nine (27\%) parents with children in the PICU group, but only two (7\%) in the GCU group, were at high risk for PTSD. Higher IES scores were reported in the PICU group than GCU group with persistent difficulties seen on the avoidance subscale (Rees et al., 2003). Findings of avoidance symptoms are consistent with studies 
of PTSD occurrence following serious pediatric accidents and newly diagnosed cancer in children (Judge, Nadel, Vergnaud, \& Garralda, 2002; Keppel-Benson, Ollendick, \& Benson, 2002).

Limitations of the study include recall bias due to interviews and data collection occurring 6 to 12 months following discharge. Although children were matched for demographics and clinical data, time from discharge to study participation is unclear. Additionally, the longer length of PICU stay could have affected study results. Researchers were not blinded to potential study participants creating a possibility of selection bias and generalizability may also be of concern as this sample was from one center (Rees et al., 2003).

\section{Delirium and ICU Psychosis}

The development of delirium in critically ill patients secondary to a general medical condition is a serious complication of the underlying disease and/or its treatment and is associated with negative outcomes (Schieveld et al., 2007; Zaal \& Slooter, 2012). ICU delirium, also known as ICU psychosis, acute confusion, and ICU syndrome (Meagher, 2009; Morandi, Jackson, \& Ely, 2009), is characterized by diffuse cognitive dysfunction, perceptual disturbances, altered sleep-wake cycles, disturbances in thought and language, and altered mood and affect. Symptom onset is usually acute with intensity of symptoms fluctuating with delirium duration (Turkel \& Tavare, 2003). Hypoactive delirium is characterized by apathy, decreased responsiveness and withdrawal with patients perceived as not thinking clearly. Restlessness, agitation, and emotional lability are exhibited with hyperactive delirium (Meagher \& Trzepacz, 2000). Patients with the hyperactive subtype are perceived to be at greater risk for self-harm, are more closely 
monitored, and administered substantial doses of sedatives and narcotics to diminish symptoms (Ely et al., 2001).

Turkel and Tavare' (2003) conducted a retrospective study to describe clinical presentation, symptoms, and outcome of delirium in children and adolescents aged 6 months to 18 years from California $(N=84)$. Children with diagnosis of delirium based on DSM-IIIR were identified through psychiatric liaisons consults. Eight clinical diagnostic categories were used to classify underlying disorders associated with delirium: infectious $(n=28)$, drug induced $(n=16)$, autoimmune $(n=7)$, post-transplant $(n=7)$, postoperative $(n=6)$, posttraumatic $(n=8)$, neoplastic $(n=7)$, and organ failure $(n=6)$.

Children ranged in age from 6 months to 18 years $(M=10.4$ years, $S D=5$ years $)$ and were predominantly male (54\%). Excess anticholinergic medication, trauma, sepsis, or postoperative status was the cause of infant delirium. No statistical differences were seen in mean age for causes of delirium based on diagnostic criteria. Children identified with delirium had prolonged hospitalization $(M=41$ days $)$ and overall higher mortality probably because of the severity of their underlying illnesses (Turkel \& Tavare', 2003).

The top five clinical symptoms of delirium in children were impaired attention (100\%), sleep disturbance (98\%), confusion (96\%), impaired concentration (5\%), and impaired responsiveness (95\%). Other symptoms included irritability, nocturnal exacerbation, affective lability, impaired orientation, agitation, apathy, anxiety, impaired memory, and hallucinations. Impaired attention in infants and toddlers was identified by difficulty in engaging them. Symptoms of attention problems in older children and adolescents were comparable to those of adults with inability to focus on one subject. Disorientation was difficult to assess in the very young child; younger, verbal children 
were asked about orientation to person and place. Altered level of consciousness was seen more often in younger children than older children (10.5 vs. 16 years) and memory problems seen more in the older child (12 vs. 9.5 years) than the younger child.

Increased morbidity, longer LOS $(M=41$ days), and a $20 \%$ mortality rate were reported in children diagnosed with delirium. The extended LOS may be explained by the higher severity of illness as inferred from the high mortality rate (Turkel \& Tavare', 2003).

A major study limitation was the wide range of participant ages making generalizability difficult. Assessing mental processing varies based on children's ages; assessing orientation may elicit appropriate responses in an adolescent while a younger child does not necessarily have any concept of person or place. Identifying clinical diagnoses associated with delirium also presents concerns in that prevalent diagnoses varies based on child's age. Additionally, identifying a toddler with impaired attention because they are unable to be engaged does not align with developmental stages of that age-group. Toddlers have fear of strangers and may not engage simply because they do not know the person.

Studying the impact of pediatric delirium (PD) on length of stay in the PICU and direct financial costs, Smeets et al. (2010) conducted a five-year prospective, observational study of children aged 1 to 18 years in a PICU in the Netherlands. Children with agitation, confusion, anxiety, discomfort, or behavioral disturbances without cause for referred by the pediatric intensivists to the child neuropsychiatrist for assessment of PD. The child was then assessed using DSM-IV criteria in collaboration with the parents and medical team about behavioral changes as well as a psychiatric examination. The child was then categorized as having PD or not. Definitive diagnosis 
was not validated with delirium instruments as they were developed for adults and are typically not used for children; a consensus meeting between the pediatric intensivists and child psychiatrist determined final diagnosis. Critically ill children who were acutely, non-electively, and consecutively admitted to the PICU and detected as having PD $(n=49)$ in routine care were compared to critically ill children without signs of PD ( $n$ $=98)$.

Measures included PICU LOS and PRISM II scores. Length of stay ranged from 1 to 62 days with a median stay of 4 days. Children with PD were older with higher rates of mechanical ventilation and higher acuity scores than those without PD. The PICU stay was significantly longer for children with PD because of mechanical ventilation and a respiratory or circulatory condition than those without PD. Children with PD stayed in the PICU an average of 2.4 days longer than those in PICU without PD resulting in additional direct costs. Severity of illness was not associated with length of PICU stay (Smeets et al., 2010).

Adult literature supports increased rates of delirium with benzodiazepine and opiate use (Ouimet et al., 2007; Pandharipande et al., 2006; Pisani, Murphy, Araujo, Slattum, Van Ness, \& Inouye, 2009); however, these were not directly examined in this study creating a major limitation. Unit policy does indicate children receiving mechanical ventilation receive sedation resulting in an indirect measure of capturing their effect. The wide age range of participants limited generalizability to specific age groups. It could be that older children were found to be more affected than younger because of the lower sensitivity of psychiatric examinations used in younger children. Confident inferences on direction of causality between longer PICU stays and increased risk of PD 
are different because a longer PICU stay increases the risk of PD and presence of delirium prolong the LOS. Children with hypoactive delirium may not have been included leading to false-negatives in the control group (Smeets et al., 2010).

Multiple studies of adults have shown significant associations between sedative administration and the development of delirium (Ouimet et al., 2007): Benzodiazepines are the most commonly implicated (Pandharipande et al., 2006). Neurotransmitter changes have been documented in delirium with variations seen in acetylcholine, gamma amino-butyric acid (GABA), dopamine, glutamine, serotonin, and histamine levels (Trzepacz, 1999). Early studies implicated the use of anticholinergic agents, including opioids and antihistamines, in reducing levels of acetylcholine producing impairment in memory and attention and exacerbating or causing delirium (Tune, Carr, Hoag, \& Cooper, 1992). GABA levels, already elevated, increase more with use of benzodiazepines leading to increased confusion, memory impairment, and agitation (Breitbart et al., 1996).

More than $90 \%$ of children in the PICU receive psychoactive medications with mechanical ventilation, most commonly a combination of opioids and benzodiazepines (Rhoney \& Parker, 2001; Twite, Rashid, Zuk, \& Friesen, 2004). Colville et al. (2008) reported that $1 / 3$ of the 102 children between 7 and 17 years of age admitted to PICU experienced delusional memories following discharge. These delusional memories were significantly associated with longer duration of psychoactive medication administration during the PICU stay. 


\section{Post-Traumatic Stress}

Research indicates significant rates of adult ICU patients suffer from PTS following discharge, with symptoms of distressing memories of the admission (Scragg, Jones, \& Fauvel, 2001) and delusional experiences such as nightmares and hallucinations (Jones, Griffiths, Humphris, \& Skirrow, 2001). Although minimal, research indicates children with PTS symptoms have high levels of distress and psychological impairment. Symptoms may persist over time resulting in long-term emotional dysfunction, adverse physical health outcomes, and poorer health-related quality of life (Landolt et al., 2009; Seng et al., 2005). Children in PICU are difficult to assess for PTS due to the nature of the environment as well as their developmental levels. PICU staff are vigilant for physiologic changes interpreting agitation, crying, confusion, anger, and aggression as well as totally opposite behaviors (detachment and withdrawal) as secondary symptoms of a physiologic event such as pain rather than PTS (Dow et al., 2012). Early PTS symptoms include nightmares, intense emotional reactions, elevated heart rate and blood pressure, exaggerated startle reflex, irritability, anger, restlessness, and sleep disturbances. Children also may appear frozen, detached, or unresponsive and may exhibit decreased awareness of their surroundings with early trauma responses (Scheeringa et al., 2011). Research indicates many children continue to experience significant PTS long past discharge (Bronner et al., 2008; Rennick et al., 2002).

In a study of 5- to $12-$ year-olds $(N=43)$ in a Canadian PICU following cardiac surgery, Connolly, McClowry, Hayman, Mahony, and Artman (2004) measured the effect of baseline cognitive level, temperament, family support, and length of PICU stay on symptoms of PTSD. Children scheduled to undergo cardiac surgery from two 
northern U.S. hospitals over the course of 20 weeks were recruited. Both hospitals had open visitation policies and all children received similar pre-operative instructions. Data collected at the preoperative visit ( 1 to 3 days before surgery) included medical, social, and psychiatric histories, cognitive assessment using child report on the Raven Coloured Progressive Matrices (CPM), temperament assessment on the School-Age Temperament Inventory, family support assessment $\mathrm{n}$ the Family Apgar measuring family support and functioning: adaptability, partnership, growth, affection, and resolve and PTSD screening. PTSD screening was conducted using parent and child report on the initial component of the Diagnostic Interview Schedule for Children (DISC) which measured child's criteria for having experienced a significant life threat. DISC responses were scored by trained personnel determining PTSD diagnosis criteria were met. The PTSD screen and interim history were repeated at the post-operative visit (4 to 8 weeks after hospital discharge). PICU LOS and SES (reflected from insurance type) were recorded from the medical chart.

Data analysis was conducted using pooled data from the two centers as no significant differences were found between subject groups. The majority of families reported private insurance (67\%). The majority of the children were Caucasian (47\%), female $(40 \%)$, with a mean age of 8.2 years $( \pm 2.5$ years); $40 \%$ were female. Baseline cognitive level was categorized as Superior (21\%), Above Average (19\%), Average (44\%), Below Average (12\%), and Impaired (5\%). Although none of the children had PTSD pre-operatively, the number of PTSD symptoms increased significantly pre- to postoperatively for the entire group. Five children (12\%) met criteria for PTSD postoperatively; length of PICU stay was the only significant predictor. No significant 
relationship was found between PTSD change score and number of previous cardiac surgeries or chronological age. The number of PTSD symptoms increased once the PICU stay exceeded 48 hours. A larger, more heterogeneous sample is necessary to compare psychological outcomes of children with previous surgeries, and varying cognitive levels and family support (Connolly et al., 2004).

Colville and Pierce (2005) investigated children's $(N=39)$ PICU memories and psychological well-being two months following their hospital discharge. In all, 23\% of the children scored above the cut point for PTSD. Children reporting delusional memories had higher PTSD scores than those reporting factual memories. Colville et al. (2008) investigated the association of PTS with delusional and factual memory in 102 children between 7 and 17 years of age three months following discharge. Principal measures were the ICU Memory Tool and an abbreviated version of the IES. More than half $(n=64)$ of the 102 children reported at least one factual memory and 33 of 102 reported delusional memories. Longer duration of opiates/benzodiazepine use was associated with delusional memory. Children reporting delusional memories had higher PTS scores, particularly on intrusive thoughts, than those with factual memories three months post-discharge. Significant predictors for PTS were emergency admission and delusional memory.

In spite of improved PICU outcomes and survival rates, between $16 \%$ and $28 \%$ of children experience deterioration of emotional well-being following PICU hospitalization (Rennick et al., 2002; Rennick et al., 2011; Rennick et al., 2008; Rennick et al., 2004; Rennick \& Rashotte, 2009) with negative emotional and behavioral sequelae commonly continuing past discharge (Melnyk, 1994; Melnyk et al. 2004). The most frequently seen 
coping responses occurring during and after hospitalization included regression, sadness, separation anxiety, apathy or withdrawal, fears of the dark and health personnel, sleep disturbances, and externalizing behaviors such as hyperactivity and aggression (Melnyk, 1994).

These studies indicate children's response to the PICU environment include intrusive thoughts and delusional memories which are significantly correlated with number of interventions, LOS, severity of illness, and benzodiazepine/opiate use with resultant PTS symptoms seen in children (Colville and colleagues, 2205, 2006, 2008; Turkel \& Tavare, 2003; Rees et al., 2003; Smeets et al., 2010). Lower cognitive levels, increased withdrawal and negative reactivity, lower degree of family support, and longer PICU stays was reported to be related to PTS in school-aged children (Connoly et al., 2004). Qualitatively, children verbalized thoughts of being scared, alone, and wanting protection.

Study weaknesses included the use of scales not psychometrically tested for children. PRISM scoring was created to reflect severity of illness in children in PICU; however, was used in children in GCU. The ICU memory tool was created for adults. Other weakness included responses being affected by data collected by the child's primary nurse or interviews being conducted in the home which could result in response bias. Generalizability to other cultural and SES groups is difficult as studies were conducted without cross-cultural or cross-SES methodologies. Unequal groups or small samples also limited power and generalizability. Study strengths were the longitudinal design using comparison groups. 


\section{Summary}

Parents and clinicians have long believed that children were incapable of understanding illness and death and any discussions with them would lead to great harm. This belief resulted in an avoidance of any discussion with seriously ill children. Results have been fairly consistent across time, indicating cognitive and psychological development affects children's understanding of illness (Myant \& Williams, 2005) and death (Bering \& Bjorklund, 2004; Poling \& Evans, 2004; Slaughter \& Griffiths, 2007; Slaughter \& Lyons, 2003), and supporting Piagetian sequential developmental stages and the concepts of illness identified by Bibace and Walsh (1980). Children's understanding of illness and causality develops from that of a global, undifferentiated perspective to one that is more concrete and abstract. Research has demonstrated that, although children progress through these stages as they age, some advance more rapidly than others (Poltorak \& Glazer, 2006).

Research findings indicate increasing understanding of illness is seen with increasing age with biological explanations more prevalent in the older child. Biological frameworks were found in children as young as 4- to 5-years-of-age without their total understanding regarding mechanisms of causality (Myant \& Williams, 2005; Zhu et al., 2009). Regardless of age however, folkloric and immanent justice rationale were used to explain illness under certain conditions supporting a coexistence model (Ramen \& Winer, 2002). Similarities in children's understanding of health, illness, and disease risks with more sophisticated knowledge seen in the older child were found in all cultures (Zhu et al., 2009). 
The child's developmental progression may be explained through increasing education from external sources which increase with age (Bares \& Gelman. 2008). More sophisticated explanations for causes of illness and their timelines was also found in children with higher socioeconomic and educational backgrounds (Zhu et al., 2009).

Research clearly indicates deterioration in children's quality of life occurs following PICU discharge (Jayashree et al., 2003; Jones et al., 2006; Taylor et al., 2003). Children experience adverse reactions such as separation anxiety, sleep disturbances, regression, hyperactivity, aggression, sadness, and apathy, (Melnyk, 2000), bad dreams, and trouble falling asleep (Muranjan et al., 2008) following their hospital discharge. Several researchers have identified children's stressful experiences while in the hospital. Any painful procedure, particularly those involving needles or "shots," is almost universally regarded as a negative experience by children (Coyne, 2006; Forsner, Jansson, \& Sørlie, 2005; Lindeke et al., 2006; Melnyk, 2000). Other stressors involve being sick, not knowing what to expect in the hospital (Carney et al., 2003), having activity restrictions and decreased independence, missing school, and being separated from family and friends (Coyne, 2006; Lindeke et al., 2006).

Children who are younger, more severely ill, and exposed to more invasive procedures are at higher risk for increased medical fears, a lower sense of control over their health, and more symptoms of post-traumatic stress (PTS) six months following hospital discharge (Rennick et al., 2002 ). Associations have been found with higher anxiety levels and length of intubation (Board, 2005); pediatric delirium and use of sedatives/analgesics (Schieveld et al., 2007); PTS and illness severity and number of invasive procedures (Rennick et al., 2004); post-traumatic stress disorder (PTSD) and 
longer duration of opiate/benzodiazepine use (Colville \& Pierce, 2005); and length of PICU stay was associated with PTSD (Connolly et al., 2004). PICU children reported higher rates of PTS symptoms than general care unit (GCU) children (Rees et al., 2004) and delusional experiences and higher rates of PTS was seen with longer periods of sedation (Colville et al., 2008).

\section{Issues and Gaps}

There is a paucity of research relating to children's PICU experiences told from their perspective and even less collected while they are still in the PICU. The lack of literature is primarily due to children's vulnerability (healthy or ill), hesitancy in approaching families due the nature and severity of the child's illness, and ethical implications relating to approaching the child and their families at this tenuous time.

Children are however, the very population we need to study as demonstrated by their risk for long-term, adverse psychological sequelae. Research in this area is challenged by the lack of psychometrically sound instruments.

The majority of the research is from parent-reports with the child heard either in data collected in conjunction with the parent(s) or not at all. Varni, Limbers, and Burlwinkle (2007) examined the feasibility, reliability, and validity of child self-report in 8591 children between 5 and 16 years of age on pediatric quality of life using the Peds QL 4.0 Generic Core Scales Database (Varni, Seid, \& Kurtin, 2001). Results indicated children as young as 5-years-old can reliability and validly self-report their health related quality of life when provided with age-appropriate instruments. While it remains important to capture the parents' perspectives, Lazarus (2000) indicated the most salient appraisal of a stressor, coping resources, and outcomes is that of the individual. 
Discrepancies were seen with preferred methodology for data collection. Ramen \& Winer (2002) included multiple choice and open-ended questions to elicit biological views of illness and quality of children's understanding of health. Children may chose explanations which include salient words such as "bugs" and "medicine" in forced choice interviews. They may also simply repeat the last option given as one they remember, or respond to the complexity of the options provided (Ramen \& Winer, 2002). Consistent with Piagetian questionnaires, open-ended interviews investigating the child's cognitive processes serve to elicit responses revealing the quality of the child's understanding and provide more meaningful responses in contrast to yes/no type responses (Bibace \& Walsh, 1980). Conversely, children, particularly preschoolers, may not have the cognitive ability to adequately express their thoughts with open-ended interviews (Myant \& Williams, 2005; Piko \& Bak, 2006). Drawing and storytelling are less directive techniques that may be useful in eliciting a child's feelings as they are non-threatening and allow the child to express perceptions they may not be "consciously aware of or able to express verbally (Bellack \& Fleming, 1996, p.10). This indicates the need to use mixed method design in order to thoroughly capture the child's understanding. Capturing children's perceptions of hospitalization using drawing methodology supports verbal and written results; children frequently are better able to explain themselves through drawings (Myant \& Williams, 2005; Piko \& Bak, 2006; Tichy, 1988). Capturing the child's understanding through use of interviews and drawing would provide much richer data.

Many of the studies are qualitative in nature using a cross sectional design. Incorporating a longitudinal design would help to capture changes over time in the child's perceptions. Qualitative studies provide rich data, particularly for children as 
participants. The addition of a quantitative measure addressing number and severity of illness is necessary to correlate outcomes based on invasiveness of care.

Multiple gaps were found relating to the state of the science for children's understanding of illness and death. Research findings have been equivocal as some studies found that children with chronic health conditions have less cognitively mature concepts of illness than their healthy peers, perhaps because of interruptions in development from illness and hospitalization (Veldtman et al., 2001). More mature concepts of illness were seen in children having asthma and diabetes (McQuaid et al., 2002). Koopman et al. (2004) found no difference in reasoning about illness between ill and healthy children.

Additional gaps included the large variations data collection points, the broad range of child ages and diagnoses, and small samples. Many of the studies lacked a true comparison group. Interventions and data collection time points varied greatly making statistical analysis for comparing outcomes challenging. Outcome measures validated for this population is lacking. Many of the studies developed their own instruments or used a validated adult tool (Colville et al., 2008; Muranjan et al., 2008; Rennick et al., 2002; Turkel \& Tavare', 2003). Much of the research is from one geographic location representing one cultural perspective making generalizability to other cultures and socioeconomic groups very difficult. A large gap remains regarding cultural and SES differences in children's perceptions of hospitalization.

Research conducted with the child while they were still in PICU was not found. Study outcomes were reported following the child's transfer to the GCU with some longitudinal studies reporting studies following hospital discharge. Although 
challenging, research should be conducted while they are still in the PICU in order to capture their continuing experience and maximize recall. This will also help to maximize child recall and decrease contamination from their GCU hospitalization. Particularly important is the need to include intubated, ventilated, sedated, and pharmacologically paralyzed children as soon as medically possible following all ethical guidelines. This population represents the most critically ill, subjected to more painful and invasive procedures. Information on these children is missing in the literature yet they represent those at highest risk for adverse psychological and behavioral sequelae which may last long past hospital discharge.

Children's appraisals and understanding of their PICU experiences are not well described. However, it is clear that children hospitalized in the PICU have more adverse responses than those hospitalized only in a GCU. Despite significant advances in health care and improved survivor rates, gaps remain in the research pertaining to the impact of hospitalization on the child's psychological, emotional, and physical responses. Critical to understanding the child's experience while in PICU is the inclusion of findings using child reports as opposed to parental reports. Additional research of psychological distress and its changes over time as well as predictors of negative psychological sequelae is needed to identify children at risk for adverse psychological outcomes. This study began to address these gaps. 


\section{CHAPTER III}

\section{DESIGN AND METHODS}

This study focused on the child's lived experience of their stay in the Pediatric Intensive Care Unit (PICU) as told through their voices. The qualitative study with repeated interviews provided data on children's perceptions of hospitalization at two time points: while in the PICU and following transfer from the PICU to the general care unit (GCU). Content analysis identified themes describing the perceptions of school-age children, adolescents, boys and girls, Hispanic and non-Hispanic, and those with differing numbers of invasive procedures. Repeated interviews allowed for explanation and comparison of perceptions following PICU admission, changes in physical condition, invasive procedures, or sedation while in the PICU, and following transfer to the GCU. Data collected from the child's hospital record included the child's age, race/ethnicity, sex, diagnosis, where they were admitted from (e.g. emergency department (ED), GCU, home, direct admission, operating room, other hospital, and physician office), length of stay, previous hospitalizations, history of chronic conditions, type and duration of sedation/paralytics, and type of insurance. Number and type of invasive procedures was collected using the Invasive Procedures Score (Rennick, 2006).

\section{Settings}

Children and their parent(s)/legal guardian were recruited from the PICU at Baptist Children's Hospital. The PICU provides comprehensive care for acutely ill children who require continual observation and monitoring. Pediatric patients' ages (American Academy of Pediatrics, 2006) range from 1 day to 18 years and patients 18 to 21 years of age if followed by a pediatrician/pediatric specialist. Patients older than 21 
years of age followed by a pediatric/pediatric specialist who are in the terminal stages of their illness may be considered for admission as a pediatric patient to maintain continuity of care.

Baptist Children's Hospital has approximately 1000 children in critical condition admitted annually with a wide range of diagnoses. Their care is provided by specialized PICU nurses, three Pediatric Intensivists, and sub-specialty physicians. The nursing station is equipped with a central monitoring system capable of monitoring all patients with bedside monitors in their rooms. The unit has eight individual glass-enclosed rooms in a U-shaped formation, allowing for constant observation, with a central nursing station. Each room has curtains for privacy. The nurse: patient ratio is typically 2:1 with 1:1 for children who are ventilated or hemodynamically unstable. A family visiting area is located immediately outside the entrance to the PICU and a family kitchen area is inside the unit. Each patient room has a television with DVD player. The PICU has open 24-hour visitation for families, including children, and a pet-visitation program. Families ( 2 members) are provided the option of being with the child during invasive proceudres and/or resuscitaiton with a family faciliator (e.g.child-life specialist, social worker, nurse, pastoral care) providing education and support

\section{Sample and Sampling Procedures}

The sample size was based on data saturation, expected to occur with $20-30$ children, from 7 to 18 years old. Using purposive sampling, about equal numbers of Hispanic/Latino and non-Hispanic/Latino children, boys and girls, and school-age children and adolescents were recruited. 


\section{Inclusion Criteria}

Inclusion criteria for the children were (a) expected PICU stay of at least 24 hours; (b) ages 7 - 17 years old; (c) able to speak and understand English; (d) able to participate in conversation (e.g. extubated, minimal sedation); and (e) in age-appropriate grade in school $+/-1$ year. Children who were intubated or pharmacologically comatose were eligible to participate after these interventions were concluded. Children who emerged from a non-induced coma were eligible to participate if they met the other study criteria. These age groups were selected because it was anticipated they would have the verbal and cognitive capacity to participate in the interview. Additionally, exploring the different age ranges provided insight into the cognitive and conceptual differences between children and adolescents relating to perceptions of their hospitalization experience. Consent from a parent/legal guardian and assent/consent from the child were both necessary for the child to participate. Custodial parent/legal guardian had to be able to read English and/or Spanish to provide consent.

\section{Exclusion Criteria}

Exclusion criteria were (a) conditions that rendered a child unable to participate verbally (e.g., cerebral palsy, severe brain damage, severe autism, comatose, intubation); (b) admission for attempting to harm themselves (e.g. alcohol poisoning, drug overdose, suicide attempt); (c) suspected parental abuse or neglect; and (d) death of a parent, sibling, or friend in the same event requiring hospitalization.

\section{Procedures}

Following Institutional Review Board (IRB) approval from Florida International University (FIU) and Baptist Health South Florida (BHSF) the PI met with nursing 
leadership and staff during PICU meetings to describe the study to them prior to study recruitment. A flyer was posted in the PICU lounge notifying staff of the study, inclusion criteria, and the PI's email and phone number.

The PICU/ Emergency Department Clinicians, Advanced Registered Nurse Practitioner (ARNP), and Pediatric/PICU Assistant Nurse Manager, serving as study key personnel, were updated daily on the status and plan of care for all patients in the PICU and pediatric GCU through daily rounding. They communicated and interacted with patients, families, and staff; provided care oversight; and facilitated patient and staff education. As part of the leadership team, they were aware of times and dates of all meetings for those pediatric areas. The PI contacted key personnel daily in person, via hospital telephone, and/or secure Baptist email to see if any patients met inclusion criteria and obtained the patient's age, ethnicity and gender. This information was necessary for purposive sampling. Key personnel identified the PICU nurse caring for the potential participant. The PI contacted the identified PICU nurse in person, via hospital telephone and/or secure Baptist email and asked him/her to ask the parent/legal guardian if they were willing to speak to the PI about the study. PICU nurses were also able to contact the PI about the parent's interest through Baptist Hospital phones and/or secure email with instructions to only state their name and contact information when contacting PI. This information was transcribed onto the enrollment log and any email communication was deleted.

If the parent/legal guardian agreed to meet, the PI approached them in the child's PICU room, asked to speak to them outside the room (e.g. PICU kitchen or lounge), explained the study using a script, and answered any questions. For parent/legal 
guardians who spoke only Spanish, Spanish-speaking key personnel (PICU Clinician and Supervisor) introduced themselves, explained the study using a script, and answered any questions. Upon agreeing to participate, the PI or the Spanish-speaking key personnel read the consent form to the parent/legal guardian. The PI and key personnel stepped out of the room for about 10 minutes to allow the parent/legal guardian the opportunity to independently decide about study participation. Parent/legal guardians agreeing to participate were asked to sign the consent for their child's participation and the Health Insurance Portability and Accountability Act (HIPAA) consent for review of the child's chart. Hard copies of both consents were given to the parent/legal guardian. The potential participant's account number was transcribed onto the enrollment log and a study number was generated.

Following parent/legal guardian consent, demographics were collected from the parent and included relationship to the child, marital status, education, age, race/ethnicity, employment, number and ages of other children, and child (subject) hospitalization history. This information was compared to medical record information and correlated with the child's responses. The PI approached the child in their PICU room about participation. The PI introduced herself to the child, explained the study using a script, and answered any questions the child had. The PI read the assent form to the children who agreed to participate and left their room for about 10 minutes allowing the child to make an independent decision to participate in the study. Children agreeing to participate signed the assent form. In the event the child was unable to write (e.g. fractures/casts, IVs), research personnel asked a PICU staff member to witness the child agreeing to participate and both signed the assent/consent form indicating on the form why oral 
assent/consent was necessary. Children were not asked to participate if the parent/legal guardian did not give consent. The parent was given a copy of their child's signed assent. Parent/legal guardians and children were told the children's data was private, parents did not have access to their child(ren)'s data, and the children were told that they did not have to talk to their parents about their study responses but could if they wanted to.

A study identification (ID) number not related to the child's hospital account number was assigned to each child. This study ID number was used on all data collections forms, interviews, drawings, and transcripts so all of the child's data could be kept together. The study ID number and the child's name was recorded on a separate enrollment log. The enrollment logs were kept in a locked drawer of the PI's locked office and were kept separate from the data collected. Only research personnel had access to these records. Electronic versions of the data were kept on the PI's passwordprotected BHSF computer and was accessible only to research personnel. Consents and assents were kept in a locked drawer in the PI's office, separate from the data collection forms, transcripts, drawings, and recordings.

\section{Interviews}

Interviews in the PICU were conducted by the PI approximately $24-48$ hours following the child's PICU admission or extubation. Interviews in the GCU were conducted approximately 24 hours following transfer from PICU to GCU. All interviews were audio-recorded on a handheld digital recording device which was stored in the PI's locked office.

Prior to each interview, a sign was put on the child's door asking staff, family, visitors to not disturb the interview session. Open-ended, conversational-style 
interviewing techniques with a semi-structured interview guide followed by probes to elicit more specific information were used to gain a full description of each child's perceptions of their PICU stay. All interviews were audio-recorded in their entirety and identified only by the child's study ID number.

The PICU interview began with the opening question "Tell me about what happened that you had to go to the hospital." Follow up questions and probes addressed:

1. What happened before you got to the hospital? The PICU?

2. What happened after you got to the hospital? The PICU?

3. What do you remember about coming to the hospital? About coming to the PICU?

4. What has it been like for you to be in the PICU?

5. What things about being in the PICU are: Good? What makes that thing good? Comforting? What makes that thing comforting? Confusing? What makes that thing confusing? Scary? What makes that thing scary?

6. Who or what: Helps the most? Makes things worse?

7. What do you think about or do to pass the time?

8. Has anyone visited you in the hospital? Who do you remember; what did that mean to you?

9. If you could magically make something about the PICU appear or stay, what would that something be? Tell me more about that thing (what it is, why should it appear or stay).

10. If you could magically make something about the PICU change, disappear, or go away, what would that something be? Tell me more about that thing (what it is, why should it go away/change, what would happen if it did go away/change? 
Prior to the PICU interview, the child's nurse was asked what medications (sedatives, analgesics) the child was currently receiving. This information was recorded on the chart review form. At the start of the interview in the GCU, each child was given a new, standardized box of crayons (8) and a drawing tablet. This allowed each child to have the same tools for drawing. They were asked to draw a picture about their stay in the PICU. Upon completion, the PI asked the child to "tell me about your drawing" with further probes inquiring about features not mentioned in their description.

The description was audio-recorded as part of the interview. The picture was archived with the appropriate transcript. Depending on what the child had already discussed in this interview, probes were used to elicit information about the machines and equipment in the PICU, types of sounds and noises the child remembered, sleeping and dreaming in the PICU, comfort or discomfort/ pain, and feelings. What types of things did you do in the PICU? What did others do to or for you? What do you remember about your parents/family in the PICU? Follow up questions addressed:

1. Tell me what it has been like for you since moving into this room.

2. What does being moved to the floor, this room, mean to you?

3. Who do you remember that visited you in the PICU? In the hospital? What did that mean to you?

4. What do you think is going to happen next?

5. When do you think you'll go home? 


\section{Data Collection Forms}

Data were abstracted from the medical records following the child's hospital discharge by the PI and a PICU nurse who served as key personnel. The study's chart review form was identified only with the child's study ID number and included the child's:

- Medications receiving at time of PICU interview

- Date of birth

- Date and time of admission to hospital, to PICU, to GCU, and discharge

- Gender, Race/Ethnicity

- Admission diagnosis and condition

- Where admitted from (ED, GCU, home, direct admission, operating room, other hospital, physician office)

- Previous hospitalizations, previous PICU admissions

- History of chronic conditions

- Sedation type/Duration

- Pharmacologic paralytic type/duration

- Equipment tubes and duration of use

- Time line of events from admission to discharge (date and time)

- Type of insurance (public or private)

\section{Demographics}

Demographics collected from the parent/legal guardian at the time of consenting included: 
- Relationship

- Marital status

- Education

- Age

- Race/Ethnicity

- Employment

- Number of and ages of children; how many live with them

- Child (subject) hospitalization history

\section{Invasive Procedure Score}

Following the child's hospital discharge, the Invasive Procedures Score (IPS) from Rennick (copyrighted 2006) was used to record the number and type of invasive procedures. The IPS list (Rennick et al., 2002) was developed as a modified version of the therapeutic intervention scoring system (Cullen et al., 1974; Keene \& Cullen, 1986, 1983 ) to record the types and number of invasive procedures children were subjected to. Invasive procedures were classified as tissue damaging (e.g. chest tube insertion) or nontissue damaging (e.g. rectal temperatures). Content validity was determined from the literature and consultation with nurse experts from one PICU and three GCUs (one surgical and two medical). A procedural checklist was used to capture the number of times each procedure was performed on the child according to using hospital records with total scores calculated for each 24-hour hospitalization period. Rennick used mean scores in her analyses and reported interrater reliability of 95 to $100 \%$ by two independent raters in reviewing 20 charts. 
The PI trained the key personnel data abstractor in this study by explaining the data collection and IPS forms, providing precise operational definitions of variables being measured, and training on how to use the instruments. Prior to actual data gathering, the first three enrolled participant charts were used to document on both data collection forms: the chart review form and the IPS for the first 12 hours of the child's PICU admission. Interrater reliability was calculated with a criterion level of agreement set at .80; any disagreements were discussed and clarified. Interrater reliability was checked every 10 charts using the chart review form and the first 24 hours of the child's PICU admission.

\section{Data Management and Analysis}

\section{Parent Demographics and Chart Data}

Sample characteristics and IPS were described with frequencies, means, and standard deviations. IPS Total scores were determined for each 24-hour period of the child's hospitalization with a mean PICU, GCU, and total score calculated for use in the final analysis. Low- and high-risk groups were determined post hoc using mean PICU and total scores with cutoff scores at the beginning of the upper quartile defining children in the high-risk group (Rennick et al., 2004).

\section{Interviews}

Audio-recorded interviews, including the child's description of the drawing, were transcribed verbatim by the PI. Transcripts were compared with audio recordings for accuracy. Each transcript was read at least once without taking notes to obtain a clear overview of the child's perceptions. Following Miles, Huberman, and Saldana (2013, pp. 69 - 105) methodology for analyzing qualitative data, text was read again and words or 
short phrases from the child's own language were used to record possible codes to detect recurring patterns related to the research questions. Similar codes were then clustered together creating a smaller number of categories, or pattern codes. The pattern codes summarized categories or themes, causes or explanations, relationships among people, and theoretical constructs. The transcripts with the codes were then entered in NVivo 10. Codes were analyzed to develop concepts which were grouped according to commonalities to create categories.

\section{Research Questions}

The three research questions answered in this study were:

1. What are children's perceptions of their PICU hospital experiences while in the PICU and the GCU?

2. How do children's perceptions of their PICU experiences change over time; from PICU to GCU?

3. Do these perceptions vary for school age children and adolescents, and for those with a higher number of invasive procedures?

Children's perceptions of their hospital experiences while in PICU and GCU were addressed using qualitative content analysis exploring concepts, patterns, and themes that reflected children's perspectives of their PICU experiences. Case summaries and codes were compared across children for PICU and GCU separately. A series of matrix summary tables were developed with child IDs in the rows and conceptually clustered codes of child perceptions in columns with each cell containing a summary of coded data in that cell (Miles, Huberman, \& Saldaña, 2014, pp. 105 - 119). The PI reviewed the data 
in a given code across cases to identify and describe major themes and explore relationships across the themes.

Changes in the child's perception over time and number of procedures were analyzed with a second series of matrices with derived codes and categories defining the matrix columns. The matrix rows were defined with child's age, race/ethnicity, sex, previous medical history (hospitalization, PICU, chronic illness), length of stay, sedation type and duration, pharmacologic paralytic type and duration, history of chronic condition, number of invasive procedures, and IPS risk group.

\section{Human Subjects Protections}

Strategies to diminish the possibility of participant identification included the use of study ID numbers, careful selection of narrative examples, nondisclosure of individual diagnoses, and alteration of specific information that could increase the likelihood of breaching confidentiality.

Consents and assents were kept in a locked drawer in the PI's office, separate from the data collection forms, transcripts, drawings, enrollment logs, and recordings. The enrollment logs were kept in a locked drawer of the PI's locked office and were kept separate from the data.

Each study participant was assigned an electronic folder which was maintained on the PI's BHSF ID and password protected desktop computer and was identifiable by the study generated linkage code. Each folder contained the transcribed interview, the electronically recorded interview, a scanned copy of the child's drawing, an SPSS file containing demographic data, the IPS, and medical record data. The file folder and all files in the folder were indexed with the child's study ID number. 
Several procedures were planned to protect the confidentiality of the data collected during this study. Data collection forms, audio-recordings, transcripts, and data files were identified by the child's study ID number only. Audio-recordings were captured digitally (no tapes) using a handheld digital recording device which was stored in the PI's locked office. Interviews were downloaded to the PI's BHSF ID and password-protected desktop computer, a USB memory stick backup copy, and a USB memory stick for transcription. Once transferred and verified that recordings were saved on the computer, recordings on the handheld digital recorder were deleted. Electronic data files were stored on a desktop computer that is also ID and password protected in the PIs office. Electronic backup copies were stored on USB memory sticks locked in a file cabinet in the PI's office.

During presentation and publication of the study's findings, quantitative data will be reported in aggregate and in a way that individual respondents cannot be identified. Likewise, the qualitative data will be presented using a pseudoname or an initial for the first name of the child. In addition, details that could identify a specific parent or child will be omitted.

Medical records are protected by the hospital as part of their day-to-day function. Key personnel collected the demographic data about the child from the medical records. The chart review form was identified only by the study-generated ID number. Identity of all study participants was confidential.

All data, consent forms, and study forms will be retained for 7 years after the last publication. At that time, paper forms will be shredded. One copy of the de-identified electronic data sets will be stored securely by the PI, locked in a file cabinet in her office. 


\section{CHAPTER IV}

\section{RESULTS}

The purpose of this qualitative study was to investigate school-age children's and adolescents' perceptions of PICU hospitalization: (a) in the Pediatric Intensive Care Unit (PICU) - within 24 to 48 hours of their being alert and able to speak, and (b) in the General Care Unit (GCU) - within 24 to 48 hours following their transfer from the PICU. The study's research questions were:

1. What are children's perceptions of their PICU hospital experiences while in the PICU and the GCU?

2. How do children's perceptions of their PICU experiences change over time; PICU to GCU?

3. Do these perceptions vary for school age children and adolescents, and for those with a higher number of invasive procedures?

This chapter presents the findings that emerged from the inductive analysis of data abstracted from children's interviews.

\section{Description of the Sample}

A total of 22 participants from 7 to 18 years of age were recruited from the PICU. Two children did not want to be interviewed following consenting and were excluded from the study. The final sample was 20 participants: 7 school age children $(5$ [71\%] between 7 and 9; 2 [29\%] between 10 and 12) and 13 adolescents (8 [62\%] between 13 and 15; 5 [38\%] between 16 and 18). More than half were male, Hispanic or Black nonHispanic, and admitted from the Emergency Department (Table 1). Eleven participants (4 school age children and 7 adolescents) had previous hospitalizations; for 2 school age 
children and 7 adolescents, the previous admission included a PICU stay. Four participants had multiple GCU admissions. Admission diagnoses were respiratory/status asthmaticus $(n=6)$, chest pain/myocarditis $(n=3)$, neurological $(n=3)$, mediastinal mass $(n=1)$, spinal fusion $(n=1)$, subdural hematoma/femur fracture $(n=1)$, splenic cyst $(n=1)$, Crohn's disease/ileostomy $(n=1)$, diabetic ketoacidosis $(n=1)$, sleep apnea/obstruction $(n=1)$, and pneumomediastinum $(n=1)$.

Length of stay ranged from 25 to 232 hours in the PICU and 13 to 168 hours in the GCU (Table 2). One school age child and one adolescent were discharged from the PICU after 232 and 46 hours, respectively, resulting in 18 participant interviews from the GCU. Two children required intubation, one school age child for a procedure and one adolescent for surgery, for 15 minutes and 75 minutes, respectively. One school age child and three adolescents received continuous infusions of sedation/narcotic medications. One additional adolescent received a continuous infusion of insulin.

The Invasive Procedures Scale (IPS; Rennick, 2006) was used to collect data from the children's medical records on the number and types of invasive procedures, both tissue-damaging and non-tissue-damaging, the children experienced. The IPS scores ranged from 2 to 106 for school age children and 4 to 58 for adolescents. As suggested by Rennick (2004), these scores were subsequently categorized by quartiles (1-6, 7-12, 13-25, 26-106) into four groups. Half of the participants experienced 12 or fewer invasive procedures. The children with the two highest scores were admitted with a complex neurological diagnosis (IPS $=106$ ) and diabetes (IPS $=58$ ). A larger proportion of school age children than adolescents were in the group with the highest number of invasive procedures (Table 2). 


\section{Table 1}

Description of PICU and GCU Participants

\begin{tabular}{|c|c|c|c|}
\hline Characteristics & $\begin{array}{c}\text { Total } \\
\text { Sample } \\
N=20\end{array}$ & $\begin{array}{c}\text { School Age } \\
\text { Children } \\
n=7\end{array}$ & $\begin{array}{l}\text { Adolescents } \\
\qquad n=13\end{array}$ \\
\hline Age in Years $M(S D)$ & $12.7(3.47)$ & $8.4(1.62)$ & $14.9(1.32)$ \\
\hline Gender $\quad n(\%)$ & & & \\
\hline Male & $13(65 \%)$ & $4(57 \%)$ & $9(69 \%)$ \\
\hline Female & $7(35 \%)$ & $3(43 \%)$ & $4(31 \%)$ \\
\hline \multicolumn{4}{|l|}{ Race/Ethnicity n (\%) } \\
\hline Hispanic & $10(50 \%)$ & $2(29 \%)$ & $8(62 \%)$ \\
\hline Black non-Hispanic & $7(35 \%)$ & $4(57 \%)$ & $3(23 \%)$ \\
\hline White non-Hispanic & $3(15 \%)$ & $1(14 \%)$ & $2(15 \%)$ \\
\hline \multicolumn{4}{|l|}{ Admitted from: } \\
\hline Emergency department & $11(55 \%)$ & $5(72 \%)$ & $6(46 \%)$ \\
\hline Another hospital & $6(30 \%)$ & $1(14 \%)$ & $5(39 \%)$ \\
\hline Operating Room & $3(15 \%)$ & $1(14 \%)$ & $2(15 \%)$ \\
\hline
\end{tabular}


Table 2

Pediatric Intensive Care Descriptions

\begin{tabular}{|c|c|c|c|}
\hline & $\begin{array}{c}\text { Total } \\
\text { Sample } \\
N=20\end{array}$ & $\begin{array}{c}\text { School Age } \\
\text { Children } \\
n=7\end{array}$ & $\begin{array}{l}\text { Adolescents } \\
\qquad n=13\end{array}$ \\
\hline PICU LOS in hours $M$ (SD) & $68.2(55.79)$ & $116.0(90)$ & $48.6(19.2)$ \\
\hline PICU IPS $M(S D)$ & $21.1(24.63)$ & $32.4(35.49)$ & $15.0(14.70)$ \\
\hline \multicolumn{4}{|l|}{ PICU IPS Group $n$ (\%) } \\
\hline $1: 1-6$ & $6(30 \%)$ & $1(14 \%)$ & $5(39 \%)$ \\
\hline $2: 7-12$ & $4(20 \%)$ & $2(29 \%)$ & $2(15 \%)$ \\
\hline $3: 13-25$ & $5(25 \%)$ & $0(0 \%)$ & $5(39 \%)$ \\
\hline $4: \geq 26$ & $5(25 \%)$ & $4(57 \%)$ & $1(7 \%)$ \\
\hline
\end{tabular}

\section{Supportive treatments $\boldsymbol{n}(\%)$}

Airway Support

$\begin{array}{lccc}\begin{array}{l}\text { Continuous Positive } \\ \text { Airway Pressure (CPAP) }\end{array} & 1(5 \%) & 1(100 \%) & 0 \\ \text { Endotracheal Tube } & 2(10 \%) & 1(50 \%) & 1(50 \%)\end{array}$

Drips

$\begin{array}{lccc}\text { Insulin } & 1(5 \%) & 0 & 1(100 \%) \\ \text { Diprivan } & 1(5 \%) & 1(100 \%) & 0 \\ \text { Hydromorphone } & 3(15 \%) & 0 & 3(100 \%) \\ \text { sure Monitoring: Arterial } & 2(10 \%) & 0 & 2(100 \%)\end{array}$


The 20 PICU interviews were conducted using core questions and probes (Table 3). Interviews conducted following transfer to the GCU began with the children drawing a picture depicting their PICU stay (Appendices A - K). They were asked to describe their pictures. Probes were used to elicit additional information about equipment and machines in the PICU, types of sounds and noises they remembered, sleeping and dreaming, comfort or discomfort/pain, and feelings. Additional probes included what types of things they did in PICU, what others did to them, what they remember about their parents/family in the PICU. Follow up questions addressed what it has been like since moving to the GCU; what being moved to the room means to them; who they remember visiting them and what it meant; what they think is going to happen next; and when they think they'll go home. 


\section{Table 3}

\section{PICU Interview Questions}

Opening question about the child's hospitalization:

- "What happened before you got to the hospital? The PICU?

Follow up questions and probes:

- "What happened after you got to the hospital? The PICU?"

- "What do you remember about coming to the hospital? About coming to the PICU?"

- "What has it been like for you to be in the PICU?"

- "What things about being in the PICU are: Good? What makes that thing good? Comforting? What makes that thing comforting? Confusing? What makes that thing confusing? Scary? What makes that thing scary?"

- "What do you think about or what do you do to pass the time?"

- "Who or what: Helps the most? Makes things worse?"

- "If you could magically make something about the PICU appear or stay, what would that something be? Tell me more about that thing (what it is, why it should appear or stay)."

- "If you could magically make something about the PICU change, disappear, or go away, what would that something be? Tell me more about that thing (what it is, why it should go away/change, what would happen if it did go away/change." 


\section{Perceptions of PICU Hospitalization}

Using an inductive approach, transcripts were read, quotes highlighted, and distinct concepts and categories were identified forming initial codes. Transcripts were entered into Atlas.ti 7 for analysis. This iterative process resulted in a total of 1520 quotes $(\mathrm{PICU}=927$; GCU $=593$ ). The initial coding schemes corresponded with the primary focus of the study (children's perceptions of PICU and GCU hospitalization) and were modified as various themes and patterns emerged. Words, expressions, or chunks of data were grouped together to represent common phenomena. Similarities in themes, ideas, and patterns that were found in the interviews were coded using the same codes. New themes, ideas, and patterns were assigned new codes. In the event different terms were applied to the same concept, the best label was used as the code name (Holloway \& Wheeler, 2010). After multiple reviews and re-readings of the various data, a total of 27 codes (subthemes) were identified. Codes were then collapsed and clustered into five themes (families) based on commonalities seen in the analysis. Themes were identified as Coping Strategies, Environmental Factors, Stressors, Procedures/Medications, and Information. Rank order of themes differed slightly based on interview location with Coping Strategies having the highest number of quotes in PICU, and Environmental Factors in GCU. Unit Portrayal was identified as a theme for descriptions of the children's drawings with 139 quotes.

Research Question 1: What are children's perceptions of their PICU hospital experiences while in the PICU and the GCU?

\section{Perceptions in PICU}

Themes, rank ordered, were Coping Strategies, Environmental Factors, 
Procedures/Medications, Stressors, and Information.

Coping strategies. Components of this theme include comforting/helped, social support, behavioral distractions, sleep, dreaming, spirituality, and trust/confidence. A total of 314 quotes were identified for Coping Strategies.

Comforting/helped. Presence of family and parents, particularly mothers, was identified by many children, regardless of age group, as a major coping strategy and social support. Parents were seen as confidantes and companions and as assisting with basic needs such as bathing, toileting, or feeding. Many children appreciated their parents, or another family member, being with them.

My mom being by my side...[she] helped me a lot. We're very close. She's kind of like my best friend too. When I first got here, I started thinking, "It's going to be alright. Just touch mom."

My parents being there, 'cause I'm not alone and I have someone to talk to, and like, they pep-talk me a little bit to getting better.

It's comforting to know that you're not..., I have my parents here to help me...I don't have to do this alone.

I have someone watching me, whether it's my sister, my grandmom, my dad, my mom, my stepmom, my stepdad, one of those. My mom cause she's been with me a lot of times. She's there by my side helping me, saying "it's okay, it's all good."

Conversely, two adolescents described not being able to discuss their own fears or anxiety as they knew their parents were anxious or scared. Consequently, the children avoided discussing their own feelings to avoid adding to their parents' fear and anxiety.

My parents were here for like, ever but then they went out to get like, some rest and get some food, which is good for them 'cause I saw they were really, really tired... Guess they were a little bit more nervous than I was...We don't really talk about it but I can just tell that they were like, really anxious... she [mom] trusted the nurse and stuff but my dad, like, he wouldn't let me out of his sight and stuff like that...I mean I would've felt fine without him but I think it made him feel 
better that he stayed.

Nurses were also viewed as helpful/comforting as well as a source of social support. Nurses were described as gentle, nice, fast, checking and being there for them. Children spoke of nurses giving them things they needed (medicines) and providing information and explanations. Both age groups described medications, IVs, aerosol treatments, and oxygen as helping them feel better.

Two children described nurses teaching them diversionary techniques, such as counting to three or using guided imagery. This was identified as a valuable coping strategy. Many children cited nurses telling jokes and using humor in their care as making them feel more comfortable, as if the nurse knew them personally.

You have like, humorous people come in and out to see you. Like the nurses are humorous. They know how to make you laugh and try to take you out of your misery.

Social support. Many children cited parents and family as social support. The value of friends was consistent across children as a source of social support. Personal cellular (cell) phones were used by the majority of children to maintain contact with their friends. Texting was seen as an integral method of communication; one adolescent was frustrated with the lack of cell service in his room and his inability to maintain contact with his friends. Many adolescents emphasized the value of friends visiting over family visiting, stating it served as a means to maintain contact with their outside world.

I think it's like, really important because like, even if you're with your parents or something, you still want to talk to someone that you have fun with and like, ease your mind and like, get to know what's going on when you're not, while you're in the hospital.

...like, I'm not focusing on how much, how uncomfortable I am or how much I'm hurting. Like, I'm interacting with my friends. I think it's better if it's friends 
[who visit]. 'Cause when it's family, they keep repeating the same thing. Over and over the same like, "Don't worry, it's going to get better." But if it's your friends, they make jokes; you'll laugh with them. But, like, one of my friends, like, they brought a present and so that, that helped me become a little happier.

Behavioral distractions. The most reported behavioral distraction was watching TV and playing video games. Children reported watching age appropriate channels, frequently cartoon network, animal planet, or sport shows. Other distractions included eating, sleeping, reading, and using social communication (e.g. Facebook, Instagram, texting). Several of the children described eating as a diversion. They enjoyed the menu and using the hospital phone to order food. One school age child cited playing with a sticker book given to her by a nurse and two adolescent males read books.

Sleep. Children described sleep as a coping mechanism for boredom. They also identified the need to sleep from illness or sleep deprivation. Conversely, children described not being able to sleep because of pain, being awakened for procedures/medications and automated blood pressures, or environmental conditions such as an uncomfortable bed and lighting.

'Cause I don't really like sleeping with all of this. I get sweaty under here [BP cuff]. And on my legs with those things [sequential compression device], I get sweaty and it's just really uncomfortable.

The only really bad thing is the light right here, and at night all you see is like, the light from the doors. Like this room will be dark but it would...the whole windows would light up the room so it wasn't dark. Like the lights would be off but it would still be bright in here.

The pain - like when I first got diagnosed, I could fall asleep even though it was bright in here but the pain now is what makes me not fall asleep. Like, I wake up. Like, I fall asleep for like, probably about 15 - 30 minutes and then I wake up.

One school age child described not being able to sleep because her family, primarily her mother, woke her asking her questions. 
I can't sleep as much. 'Cause they're always doing something or asking me questions. 'Cause of my family.

Spirituality. A male adolescent described how holding his head up and keeping his faith helped. An adolescent viewed her faith as a strong sense of support. Her mother would pray with her, immediately calming her.

I would go with the praying, but not everybody is you know. But I never give up on my faith and I never keep my head, I always keep my head up high. I always put things in His hands and whatever happens, happens.

Trust/confidence. A school age child described his loss of trust and confidence in his father. He cried as he told of his sense of betrayal when he was taken to the operating room subsequent to his father telling him he was not going to have to be put to sleep for surgery. An adolescent described his trust in the hospital personnel stating, "They told me everything's going to be okay and I believe them."

Environmental factors. Components of this theme include equipment, noise, unit differences, hospital personnel: positive, self-determination, change, food, hospital personnel: negative. Two hundred thirty eight quotes were identified.

Equipment. Many of the children spoke about the equipment, referring to electrodes as wires, little stickers, or stuff. Most were not able to describe the monitor's function stating they were not really sure what it did. Some children used colors to describe the wave patterns on the monitor screen. Most children understood the blood pressure, viewing it as an annoying necessity. The majority of the children stated they were not bothered by the equipment. The oxygen saturation probes on the children's fingers also were not clearly understood; some said it told them how their finger was doing. The pressure from the probe caused discomfort for the children. 
Hospital personnel: positive. Nurses, physicians, and dietary personnel were identified as staff who were helpful, friendly, and cheerful. Children described liking nurses who checked on them, asked how they were doing, and were attentive to their needs. Two adolescents stated nurses respected them and made them feel at home.

The attention you get from the nurses. They're really attentive and you feel like, they're close and really polite. They look out for you. They ask if you're in pain. They help out a lot with a lot of things.

From the nurses. And when I've called them to like, about the medicines or whatever, they immediate, they immediately like, called the pharmacies or whatever. Like, they'll get me the medicines. When I need to go to the bathroom, they don't take forever. They're on point all the time.

Change. When asked what the children would change about PICU, many of them stated they would make the event not happen, to stop being sick or not feeling well, for the pain to be gone, and to not have surgery. Some addressed the sense of isolation they experienced and described PICU as a big separate unit with no contact. Two adolescents wanted to change the PICU's name as "Intensive Care Unit" evoked fear. A school age child stated she would have "the doctors find the IV and make children feel better." An adolescent described how overwhelming the equipment appeared to her, particularly when she was first admitted. She wanted the machines to be smaller so they would not seem so scary.

Many children addressed environmental changes they would make to the room. Two adolescents described not liking the bed, finding it too hard. All other children liked the bed, found it soft, and especially liked playing with the buttons. Some addressed wanting a view, wanting to see the sun whereas an adolescent with a head injury felt it was too bright and wanted less light. The lack of showers and being able to bathe, 
feeling dirty, was described by two adolescents. Two school age children wanted the room to be painted and decorated while one adolescent liked the neutral colors in the room as it reminded him of his room at home.

I would put flowers all over (pointing to the ceiling) and then paint it pink. The floor would be nice and shiny. Those windows, those would, would be uh, they would be a little bit taller. I would make everything the way, the way I wanted it. I would want it decorated.

Noise. Children described noises heard in the PICU with "beeping" as the most frequently identified sound. Many of the children understood the reason for the noises, particularly IVs, and noted the sound levels were turned down at night so as not to bother them. Many of the children stated the noises didn't bother them at night, they were used to noise, and the noise did not affect their sleep. An adolescent felt the PICU was too quiet and too isolated. Two children described weird noises and were unable to identify the source. One school age child seemed concerned about the weird "swish" he would hear.

It would be, like the, a weird swish. Like um, when everyone steps out for a second and there's no one else in there except for you, even if it's for like five minutes, you hear, like a little swish and it's so weird. It doesn't stop. It's only when everyone's out.

Self-determination. Many of the children addressed frustration regarding their lack of control with their treatment, surroundings, and privacy. They spoke of being strapped down, trapped, and confined to a bed or a room. They addressed not having freedom with their hands and arms because of IVs and the annoyance of being hooked up to machines. They expressed frustration that they were not permitted to leave the room to just walk around outside. Males in particular addressed wanting to be outside, not confined, playing sports. Two children, one school age child and one adolescent, 
discussed their lack of privacy.

Before I was like really, like I don't want other nurses to see my private parts and me naked, but after a while I was so fed up I was like, whatever, who cares. Just do what you have to do. If my gown's not really all the way down, if it's like up here, 'cause it's like, I have to move around a lot and I have to get up and they're here and they like, took off the blanket and they see you.

The school age child describing the lack of privacy had a severe neurological condition with tremendous speech difficulty. Although it took several minutes for him to verbalize his thoughts, he wanted to ensure they were conveyed. He eventually tired and motioned to the curtains.

Well, when I want to go...I want to go, um, I want to go pee, the curtains are sometimes, well, sometimes, um sometimes...

Food. The majority of the children liked the menu, the food, and the ability to use the room phone to order. Many of them addressed looking forward to eating following procedures or medications. Pancakes, hamburgers, and broccoli were cited as the best foods. Two school age children and two adolescents stated the food was the best thing about the PICU, "I like the food more than anything." One adolescent male felt it was not very nutritional, tasted like filler food, but there "was plenty."

Hospital personnel: negative. Negative perceptions for hospital personnel included delays in care, speaking in medical jargon, or inattention to detail (not closing an ileostomy bag). The adolescent with diabetes described his frustration with being extremely hungry and having to wait for his insulin injection following his blood glucose check and then waiting longer for his food. A school age child described how he frequently did not understand what the nurses and doctors were saying; he would ask his mother for clarification. 
Unit differences. Two adolescents identified moving to the GCU would mean they were getting a little bit better but did not really understanding how care would differ. One adolescent verbalized he did not think there would be any differences between units for him as he would still be confined to bed.

Stressors. Components of this theme, with 143 quotes, include pain, fears/scary, frustration, time, and separation.

Pain. Children addressed various stressors created during their PICU hospitalization. Pain was the most frequently described stressor - pain from their admission diagnoses (e.g. myocarditis, skull fracture) or surgical procedures.

The lights, the lights. Especially that light [wall]. That light. Oh my God, it kills because my eye, like this eye here, since it's bruised and things like that, since that's the side I hit myself in. It hurts every time like I look up and that light, oh my God.

Children described pain from tubes being removed, IV medications, being sore from breathing so hard, and pain from coughing. One adolescent described the severe pain of an IV infiltration. Some adolescents described visitors as increasing their pain because of the visitor's talking or moving. Most recounted narcotic administration or environmental changes (lighting) decreasing their pain and subsequent ability to sleep. Some described perceptions of suboptimal pain management.

That night I was screaming in pain. They didn't give me enough medicine to stay asleep for the rest of the day. I was literally shaking and screaming that night. You're very drugged but as the medicine starts to wear off, you start to feel it. Like, I felt it. And I was like, shaking and screaming, and all I could think about was being brave.

I was in a lot of pain, and, um, I had to use the bathroom and it took me a long time to get up from the bed to the bathroom. They gave me the, that [pointing to the patient-controlled analgesia pump] where you just press the button and it gives me medications. And like, the pain goes away but not completely but it's a, like, 
a little bit better. It helped a little bit but not, like, too much. I still had the pain but it made it a little bit better.

Fears/scary. Some children, regardless of age group, were very forthright about being afraid or scared. Two adolescents addressed their fear of surgery, although surgery was not anticipated for their diagnosis. Some identified being afraid of the radiology procedures and both age groups addressed fear of needles or being stuck. The "ICU" caused fear from its name alone.

The word "ICU, Intensive Care Unit" is such a frightening and terrifying word when you first hear it, because you think, oh my God this is so severe. It's a scary thought when you say "Intensive Care Unit" because you think, like you see the monitors, you think everything is so severe. When I found out I had to go there, I was so scared. Because if you think about an ICU, you think pain.

One school age child addressed being afraid of the shots the doctors were going to give her when they came in. Upon further exploring, she was not expecting to receive any more IVs or shots.

I only remember the doctors when they come in and stuff. The stuff that they have. Shots.

Frustration. The children described frustration with being hospitalized over the summer and ruining their plans. Some did not understand their diagnosis and plan of care feeling frustrated over the duration of the hospitalization. An adolescent admitted post motor vehicle crash expressed extreme frustration with not being able to walk, nurses not being able to get the IV immediately, the need to repeat lab tests, not being able to leave the room, and the sense of isolation.

I think I was just crying out of frustration. 'Cause I don't want that to happen. And it's the beginning of summer and now I have to be here. For a long time. It was frustrating 'cause, like, in my mind I was like, they're nurses. They should be able to pinpoint a right vein for that. And it hurt. And then when they did it on this one, they have to do another IV, so I was pretty frustrated. I've been pretty 
frustrated like, the whole time. It makes me feel frustrated cause, like, they have to do the same test over and over. And they have to take blood from this arm and my other arm. I've done a lot better but it's like, I'm not comfortable. I'm not $100 \%$ or close to it. I'm so like, frustrated. Frustrating. Like, I have to be in this bed the whole time.

Time. Time was identified as an additional stressor. Children described how they lost track of time in the PICU and were unsure of how long they had been hospitalized. They spoke of how slowly time went and one child reporting even sleeping very slowly. One adolescent described losing the concept of time.

I just see like, the clock change, but I can't see like, outside where it is and I would, like, text my friends this morning, but to me it felt like afternoon or evening but to them it's just morning.

Separation. Children spoke of separation - missing family, friends, and pets. One adolescent professed to feeling he let down his friends by not being able to help them with a fund raising event.

Procedures/medications. Components of this theme include IVs, procedures, medications, and laboratory (lab) with 146 quotes.

IVs. Children understood IVs were necessary, providing medications and fluids. Many of them referred to them as shots. They spoke of multiple IV attempts because of poor veins or veins collapsing. The process of starting an IV made them feel woozy, bad, or hurt - only when being started. One adolescent described his veins being hard to find and how getting tense makes it harder to find the vein. A school age child offered the advice of not moving when they do it and it may only [hurt] a little. A post-operative school age child spoke of frustration with the IV experience. 
They were sticking me with IVs. A lot. It hurt, bad. [I told them] no more. It's been three days and they have to switch it. Really, like I have to do this one more time? I know that's a new record. Because I've never been sticked that much times.

Procedures. Children described their procedures using words such as "brain test", "scanner of my heart like they do with pregnant ladies," or "esophagus test." One adolescent described greater anxiety when a test had to be repeated fearing negative results.

The x-ray. It's like these things that they take pictures of you while putting these clips on. But they had to do it twice because the first one came out weird. So they had to do it twice and I had to do it again when I came back. [I was] worried actually, since they said the first one didn't come out so good so they had to do it again.

A school age child described intense fear when the nurses moved or checked the ileostomy bag. A post-operative adolescent described removal of the chest tube. A school age child thought she was being changed to another PICU room each time she went to a procedure. Three adolescents described going to the pre-operative area, had total recall of medication given to relax them, and not remembering anything until they awoke in PICU. An adolescent recovering from surgery identified a sense of security when needing to stand for x-rays.

I had an x-ray done standing up so that was, that was a weird experience because it was the first time I really walked and I moved around and they had me positioned. They will always have a couple of people around you to hold you in case you start feeling pain or get dizzy.

Medications. Children primarily described medication given to them orally or aerosolized recognizing it helped them feel or breathe better. One school age child and one adolescent verbalized pain when IV medications were infusing. They said the nurses treated the pain by either slowing down the infusion or applying warm packs. 
Lab. Children also expressed frustration with lab work. One adolescent stated, "I know it's necessary but it's just annoying to get stuck with needles all the time." They explained sometimes blood was obtained from their IVs, veins, or finger sticks. An adolescent stated that watching them draw blood made her feel like she was going to faint. Children understood that they needed the lab work but were frustrated with being stuck to obtain the blood.

Information. Components of this theme include thoughts/feelings, advice/expectations, and knowledge with a total of 86 quotes.

Thoughts/feelings. Children expressed reasons for their hospitalization and their thoughts prior to being admitted. One adolescent recounted thinking, "Something was pretty serious, but I just realized they were having me here to monitor me since I just had major surgery." The adolescent went on to express disappointment with missing the first summer job as a lifeguard due to hospitalization. Another verbalized feelings over missing the entire summer as a result of a femur fracture; being transferred to the PICU from the rehabilitation unit accentuated the disappointment. This child stated, "When you're alone and you think, 'Why am I here' and all that; the room gets smaller and smaller. You just want to start crying."

Advice/expectations. Children were asked what advice and/or recommendations they would give to their friends if they needed to be admitted to PICU. The majority of children stated they would tell them to not be scared, it's a good place, you'll be treated well, and there is entertainment. They also would tell their friends to stay positive and bring something to do as even TV gets boring after a time. One adolescent suggested talking to the health care providers. 
Talking to your nurses and doctors more so they would help you understand what's happening and uh, like, how they help you feel better faster.

Another adolescent would tell them they are going to be frustrated and mad. The continuous bright lights and difficulty sleeping was addressed by another adolescent. A school age participant would warn friends about the sticks and IVs.

Knowledge. Many children were able to describe reasons for admission and treatment. Two adolescents cited their surgery and plan of care and one school age child described frequent vomiting and inability to walk as the need for hospitalization. The majority of the children did not know what the cardio/respiratory monitors were or their function.

\section{Perceptions in GCU}

The interview in GCU began with the children drawing a picture depicting the PICU experience and then describing it. Although they had 8 different colored crayons, many of the children used only the black crayon drawing the basic equipment in the PICU and did not include people in the picture. One adolescent drew a person in the bed without a head, wanting anyone who looked at the picture to be that person and be able to visualize the room. Children described being able to see other hospital rooms [Adult ICU] from their windows but not the people in them. Two described babies crying in other PICU rooms.

One adolescent drew a cell phone, watching the World Cup, and balloons that friends had brought. This adolescent depicted [self] crying, stating this was the adolescent's mood the whole time while in the PICU; not actually crying but feeling that way. Another adolescent also drew visitors watching the World Cup on TV with her. 
That adolescent was laughing and joking the whole time. Some of the children who drew themselves in the PICU depicted their mood as happy; only one child drew his face as sad. A school age child drew two clocks with two different times in the picture of the PICU.

Quotes and themes were less in frequency in the GCU interviews; however, the children's GCU interviews typically indicated perceptions consistent with the PICU interviews. Themes about the PICU, rank ordered, varied in the GCU interviews from the PICU interviews, with Environmental Factors being first (218 quotes), followed by Coping Strategies (190), Stressors (94), Procedures/Medications (52), and Information (39).

PICU environmental factors. In the GCU interviews, components of this theme include equipment, hospital personnel: positive, noise, self-determination, food, hospital personnel: negative, and unit differences.

Equipment. In the GCU interviews, similar to their PICU interviews, children addressed equipment and monitors to which they had been attached. Some knew the purpose of the equipment, while others admitted not knowing. They addressed the automatic blood pressure, how frequently it inflated, and how annoying it was. They described the IV, the TV, and the sofa [GCU] which two of their mothers discovered opened to a couch following their child's transfer. Many of the children described their GCU view, the ability to see outside, and how good the sunshine and light made them feel.

Hospital personnel: positive. During the GCU interview, the PICU nurses were described as being on top of things and telling jokes. One adolescent described the 
[PICU] nurse as having the "same problem that I did so she was like in common; she knew what I was going through.” Two adolescents discussed the PICU nurses speaking to their parents, keeping them informed.

Noise. Many of the children in the GCU interviews addressed the beeping of the machines in the PICU, stating they were not really bothered by them, and the nurses would come to fix them. Many of the children stated the beeping meant the IV fluid or medication was complete. Babies were heard crying [PICU] but it was not seen as disturbing. Bathroom noises were described by two children as the bathroom was shared between 2 PICU rooms.

All the time, 'cause there was pretty much people coming in - 'cause that room shares a bathroom with the other room. And they kept coming in, and they'd lock the door from the other entrances, they'd lock it. And then there's flushing, flushing, flushing.

Noise from nurses talking outside PICU rooms was described by two adolescents.

When I was sleeping, you can hear like, the people talking outside. And you can also hear like, other people's machines. Um, you heard my machine, the TV, um, it's not uncomfortable but just kind of bothered. The nurses like, let's say they laughed really loud, I could hear that.

Self-determination. Several adolescents remembered their lack of control over their privacy in the PICU, particularly when going to the bathroom to urinate. Children discussed the lack of freedom because of being hooked to monitors or confined to a room or bed.

Food. Children were consistent regarding perceptions of the PICU food. They liked it and felt a sense of empowerment being able to order food themselves. One adolescent noted an increase in appetite as the adolescent's respiratory status improved. 
Hospital personnel: negative. A school age child described the PICU nurse leaving the child's ileostomy bag open so "stuff got all over [the child]" when getting up to walk the first time. This same child in the PICU interview recounted fear with anything pertaining to the ileostomy bag.

Unit differences. Many described differences in the room size, the view from GCU large windows, and the lack of computers. They described their GCU rooms as being prettier, yellow and blue, with fish on the walls. They were now free to move around and leave their room. One adolescent addressed the change in nursing care between the units, saying the care was excellent despite the GCU nurses having more patients. The majority equated moving to the GCU as getting better, one step closer to going home.

Coping strategies. Components for this theme include comforting/helped, social support, behavioral distractions, sleep, and dreaming.

Comforting/helped. Children in GCU interviews remembered parents, particularly mothers, friends, and nurses as sources of comfort, help, and social support in PICU. Most children described their parents alternating who stayed with them and their needing to go home to take care of siblings or to go to work. They recounted how too many visitors in PICU increased their levels of pain and anxiety. Friends provided distraction different than family.

Because my friends were there for only like, an hour or so, so like, I would want to cherish that moment as much as I can. My parents are there the whole time so it's not the same thing. 
Nurses in PICU were again remembered for providing humor. Different from PICU interviews, children in GCU interviews verbalized well-loved items providing comfort. When asked what was helpful or comforting in the PICU, a school age participant described how stuffed animals provided comfort. An adolescent described how comforting a familiar nurse in PICU and the blanket that the adolescent always keeps close were.

The stuffed animals. I had one from my house. Her name was Pixie. It's a LaLa Loopsie doll. 'Cause she always makes me feel better. She's right here.

One of the nurses that I knew, that I had her here [in the GCU] when I first got diagnosed. Um, she made me comfortable. 'Cause I know her....My blanket which is under here. I used to bring my little pillow when I first got diagnosed but I lost it here, so...I had that pillow since I was born.

Social support. Children in GCU interviews described family and friends as their primary source of social support in PICU saying that it demonstrated they cared and provided distraction. Two adolescents spoke of how visitors in PICU increased their pain levels. Children expressed happiness when they were visited by people they were not expecting such as teachers or a manicurist.

Behavioral distractions. As in the PICU interview, children in GCU remembered talking with relatives, watching TV, sleeping, and texting with cell phones in the PICU. These activities continued as the majority of behavioral distractions for the children on the GCU.

Sleep. Sleep in the PICU was identified in the GCU interview as something children did out of boredom or illness. They described being awakened by pain or the inability to get comfortable because of all the equipment. 
I couldn't move around so much. I had like 4 or 5 wires on this side, or my left side. And one wire on my right side. So it was hard for me to sleep. And I couldn't sleep on my side.

Two adolescents described taking advantage of narcotic administration in PICU to catch up on sleep. Most stated noise did not prevent them from falling asleep or wake them; however, the automatic blood pressures or nurses replacing electrodes did wake them. An adolescent described being awakened and embarrassed in PICU after hitting the nurse call button by mistake and from the IV burning. Another identified making [self] stay awake when respiratory treatments were due. Consistent with the first interview, the post-surgical adolescent described how the bright lights in the PICU kept the adolescent from sleeping.

Dreaming. Children in GCU interviews described more PICU dreams than those mentioned in the PICU interview. One school age child and one adolescent described dreams of going home. Another adolescent stated the dreams were odd and shifted a lot. One moment they were of family and the next of being in strange places. An adolescent described dreaming about being home, playing video games and being Batman. A school age child described only day dreaming.

Stressors. Components of this theme include pain, fears/scary, frustration, time, and separation. Children in the GCU interview recalled similar stressors consistent with their PICU interview.

Pain. They described pain in the PICU caused from the IV (e.g. medications or infiltration), movement, procedural (e.g. gas, surgical), or diagnosis (myocarditis, respiratory). 
The IV, kind of painful. Sometimes when I was down there [PICU] for the second time, it would burn a lot. Well not the whole time but when he took it out, it felt better. They had to swap it. It was really burning and the nurse had to get some equipment and it really, really hurt. I felt like it took 20 years - I'm just exaggerating. It was really burning. I had to get it off! I was about ready to disconnect it but I didn't know...I didn't want to mess anything up and stay longer.

One adolescent voiced thoughts about the severity and duration of pain in PICU and GCU, fearful that it would not decrease soon. Several addressed the benefit of pain medications, how quickly they worked, and how they were able to sleep following administration. One adolescent spoke to not receiving pain medication in a timely manner and how the adolescent would awaken because of the pain. Children stated they felt better and had less pain since moving to GCU as they have had a chance to heal and recover. Adjuvant pain interventions included breathing treatments for coughing, warm compresses for the discomfort of pain medication, and the use of a spray prior to finger sticks.

Fears/scary. Children in the GCU interview addressed, as in the PICU interview, fears of shots, needles, and getting stuck. In both interviews children described being afraid of needing surgery, radiology results, and surgical outcomes. One adolescent conveyed fears about radiology results.

I was afraid that the scans were going to come back with something bad or something like that but they didn't.

One adolescent spoke about fear of not having the same level of care on the GCU as in the PICU. Children on GCU described being afraid of not getting better, having longer hospitalizations, and missing school. A school age child expressed fear of shots and IVs while in PICU, indicating misconceptions of their purpose. 
Those machines [PICU] looked pretty scary 'cause I thought the medicine that went in here [pointing to IV]...they kind of look scary 'cause they kind of look like shots. They [syringes] looked like shots, they're really like shots because they had this little thing that you pulled and here comes out the water. But it's kind of different than the shots because the shots have a little needle. It was kind of scary. I thought you would go like, go in my skin and then be, really. It would hurt myself. I didn't really feel it.

Frustration. Children spoke of the frustration of being ill and not being able to get out and walk around in PICU. One school age child described frustration with family for their lack of support saying, “They're telling me stuff like I can't do it. I'm never going to get out of the hospital."

Time. Children continued to describe not being aware of the time in PICU, if it was night or day. Many of them did not know how long they had been hospitalized. One adolescent was surprised to discover it had been storming when in the PICU but had no way of knowing, not even being sure what time it was.

Separation. Two adolescents in GCU described missing their homes family, friends, and dogs. One spoke of missing his/her bed.

Procedures/medications. Components of this theme include IVs, procedures, medications, and labs.

IVs. Frequently the children described IVs, the procedure itself, frequency of sticks, number of IVs, pain they caused, and how they made them feel [bad]. The process of changing IVs in PICU and GCU was described as painful. One school age child recounted the PICU nurses telling his/her parents about starting a second IV but not the child.

There was two when I got the other one stuck in the other arm. I didn't like it. This one, they had to take it out 'cause it was hurting me. They took it out. It hurt a little but I got used to it when they were taking it out. They were talking to 
me like...The lady came in; she was talking to me, but I was crying so much while they were doing it, but it hurt. I just got one taken out.

Procedures. Children's descriptions of procedures did not vary between PICU and GCU. They spoke of having their vital signs frequently taken in PICU. They talked about x-rays and ultrasounds in layman's terms. The school age child described the anticipated removal of a tube as being contingent upon fluid intake while in GCU.

Medications. Consistent with PICU, medications were identified as helping children feel better with the majority describing respiratory treatments in PICU and GCU. The adolescent who had described his/her heart beating out of his/her chest in PICU repeated the same description in GCU.

Labs. Children recalled being stuck with a lot of needles for lab work and the pain associated with it. The GCU interview differed from the PICU interview in that they recognized they needed to have more labs or wait for results in order to be discharged. One adolescent said, "They're taking a lot of blood but I want to get out of here as soon as possible so I'll do whatever they tell me."

Information. Components of this theme include thoughts/feelings, advice/expectations, and knowledge.

Thoughts/feelings. Two adolescents described their feelings with having to be admitted to PICU subsequent to thinking they were getting better. One had been in PICU, transferred to GCU, and had to return to PICU. The second had been transferred to the rehabilitation unit from another hospital, deteriorated medically, and had to be taken to PICU. These adolescents felt they were "starting all over" with the first part of 
their admission not meaning anything. A school age child reiterated his/her love for home and how the child could not wait to be discharged.

Advice/expectations. Children interviewed in GCU said they would tell their friends to not be afraid, "They'll treat you like you're at home," and "Everything's going to be alright." Also mentioned was the need for friends visiting. They advised their friends to not be afraid of the name "Intensive Care Unit;" it just means extra special attention. The children consistently stated the personnel were trying to help and make them feel better.

Suggestions were also made for their friends to bring things to help pass the time and some of their stuffed animals to keep them company. One school age child would tell friends to expect machines they don't know about and they may get stuck a little bit.

Don't be scared because they're not there to hurt you. They're there, they're there to help you but don't be scared when they stick you and stuff because it's to make you feel better.

Children also wanted to advise their friends of the negative expectations associated with the PICU.

I could tell you that they're going to bother you a lot, like with the monitoring and stuff like that; and all the IVs and especially if you're in a lot of pain that they're going to be asking $24 / 7$ if you're ok and stuff like that. They're going to wake you up in the middle of the night to test you and stuff like that. And doctors will be coming in and out. And to have faith and you know, keep your head up high.

Knowledge. Two adolescents described post-hospital treatment indicating understanding of their plan of care. Conversely, another adolescent expressed frustration with a lack of knowledge about the diagnosis and recovery time. The adolescent felt the doctors were not telling him/her everything and were treating the adolescent for the 
wrong diagnosis. A school age child identified the need to increase fluid intake to have tubes removed.

Research Question 2: How do children's perceptions of their PICU experiences change over time (PICU to GCU)?

\section{Perceptions Over Time}

Two children were discharged from PICU resulting in a sample of 18 for GCU interviews. Overall, there was a decrease in the amount of codes by all children. An increase in responses was noted in GCU for sleep, dreaming, noise, unit differences, and advice about expectations. No responses were recorded in GCU for spirituality, trust/confidence, and change. Approximately equal proportions of quotes were seen between PICU and GCU in Coping Strategies (33.9\%, 32\%), Stressors (15.4\%, 15.9\%), and Information $(9.3 \%, 6.6 \%)$, respectively. Coping Strategies demonstrated the largest proportion of quotes (33.9\%) in PICU compared to Environmental Factors (36.7\%) in

GCU. The proportion of quotes for Procedures/Medications in PICU (15.7\%) was double that in GCU (8.8\%). Proportions of subthemes in PICU found to be more than double those in GCU, respectively, included hospital personnel: positive (21.5\%, 4.6\%), change (18.1\%, $0 \%)$, hospital personnel: negative $(2.5 \%, 0.4 \%)$, frustration $(11.2 \%, 3.2 \%)$, and knowledge $(25.6 \%, 12.8 \%)$. Proportions of subthemes in GCU found to be more than double those in PICU, respectively, included sleep (32.6\%, 13.1\%), dreaming (4.2\%, $0 \%)$, noise $(25.2 \%, 13 \%)$, unit differences $(33.5 \%, 0.8 \%)$, and advice/expectations $(59 \%$, 25.6\%). A greater proportion of quotes for pain were in GCU (58.5\%) than in PICU (44.7\%). Results of children's perceptions over time (PICU to GCU) can be seen in Table 4. 


\section{Table 4}

\section{Perceptions over Time (PICU to GCU)}

\begin{tabular}{|c|c|c|c|}
\hline Themes & PICU $(n=20)$ & GCU $(n=18)$ & Total \\
\hline Total $n(\%)$ & $927(61 \%)$ & $593(39 \%)$ & 1520 \\
\hline Coping Strategies $n$ (\%) & $314(33.9 \%)$ & $190(32.0 \%)$ & 504 \\
\hline Comforting or Helped & $100(31.8 \%)$ & $51(26.9 \%)$ & 151 \\
\hline Social Support & $88(28.0 \%)$ & $38(20.0 \%)$ & 126 \\
\hline Behavioral Distractions & $77(24.5 \%)$ & $31(16.3 \%)$ & 108 \\
\hline Sleep & $41(13.1 \%)$ & $62(32.6 \%)$ & 103 \\
\hline Spirituality & $6(1.9 \%)$ & $0(0 \%)$ & 6 \\
\hline Trust/Confidence & $2(0.7 \%)$ & $0(0 \%)$ & 2 \\
\hline Dreaming & $0(0 \%)$ & $8(4.2 \%)$ & 8 \\
\hline Environmental Factors $n(\%)$ & $238(25.7 \%)$ & $218(36.7 \%)$ & 456 \\
\hline Equipment & $64(26.9 \%)$ & $47(21.6 \%)$ & 111 \\
\hline Hospital Personnel: Positive & $51(21.5 \%)$ & $10(4.6 \%)$ & 61 \\
\hline Change & $43(18.1 \%)$ & $0(0 \%)$ & 43 \\
\hline Noise & $31(13.0 \%)$ & $55(25.2 \%)$ & 86 \\
\hline Self-Determination & $25(10.5 \%)$ & $20(9.2 \%)$ & 45 \\
\hline Food & $16(6.7 \%)$ & $12(5.5 \%)$ & 28 \\
\hline Hospital Personnel: Negative & $6(2.5 \%)$ & $1(0.4 \%)$ & 7 \\
\hline Unit Differences & $2(0.8 \%)$ & $73(33.5 \%)$ & 75 \\
\hline Stressors $n(\%)$ & $143(15.4 \%)$ & $94(15.9 \%)$ & 237 \\
\hline Pain & $64(44.7 \%)$ & $55(58.5 \%)$ & 119 \\
\hline Fears/Scary & $50(35.0 \%)$ & $29(30.9 \%)$ & 79 \\
\hline Frustration & $16(11.2 \%)$ & $3(3.2 \%)$ & 19 \\
\hline Time & $10(7.0 \%)$ & $5(5.3 \%)$ & 15 \\
\hline Separation & $3(2.1 \%)$ & $2(2.1 \%)$ & 5 \\
\hline Procedures/Medications n (\%) & $146(15.7 \%)$ & $52(8.8 \%)$ & 198 \\
\hline IV & $58(39.7 \%)$ & $21(40.4 \%)$ & 79 \\
\hline Procedures & $41(28.1 \%)$ & $14(26.9 \%)$ & 55 \\
\hline Medications & $34(23.3 \%)$ & $8(15.4 \%)$ & 42 \\
\hline $\mathrm{Lab}$ & $13(8.9 \%)$ & $9(17.3 \%)$ & 22 \\
\hline Information $n(\%)$ & $86(9.3 \%)$ & $39(6.6 \%)$ & 125 \\
\hline Thoughts and Feelings & $42(48.8 \%)$ & $11(28.8 \%)$ & 53 \\
\hline Advice/Expectations & $22(25.6 \%)$ & $23(59.0 \%)$ & 45 \\
\hline Knowledge & $22(25.6 \%)$ & $5(12.8 \%)$ & 27 \\
\hline
\end{tabular}


Children in GCU interviews described being able to look outside through large

GCU windows and see people, parrots, cars, and the sun.

The outside view. I love it. It's better to see how the outside world is. 'Cause if you're just inside, you don't feel like out there is in here.

...because of the window [GCU]. It makes a difference, it can get your mind off things... it [PICU window] was blocked by like, a building...it takes your mind off of things and has you focus more on like, the outside, like I'm going to be out there soon.

The GCU was portrayed as being more nicely decorated with prettier colors and

fish on the walls which was described as more comforting. Children were now afforded the luxury of showers and privacy for toileting.

...except the bathroom's bigger, and there's a shower 'cause I got to take a shower last night... So great, I felt as if I had a pound of dirt on me. It felt so great.

The adolescent requiring fluid boluses in PICU now described how urinating was better because of being able to walk to a private bathroom. Differences were described regarding the room size; some children felt the GCU room was smaller and others larger. An increase in mobility and freedom was described by many of the children as a difference between units. They were no longer attached to "computers" just the IV, no longer heard annoying beeping, and now had the ability to freely walk around. They could leave their rooms.

Well, for one, 'cause I'm less limited. 'Cause in the ICU I was basically limited to the bed and nothing else; that now I can just walk around. Like I finally took a shower which I wanted to.

The adolescent who was unable to text by cell phone in the PICU had cell phone service in the GCU and expressed how nice it was to keep in contact with friends. Two 
adolescents described being able to sleep better because the IV pole was on the same side as the IV. They were no longer getting tangled in tubing.

Family and friends were consistently viewed as comforting coping strategies. Some of the children described having more friends visit and less restriction when their friends are there.

All children, regardless of age group, felt the transfer indicated they were getting closer to being discharged, one step closer to home.

That I was getting better. 'Cause it was like, because I can come up here now because I've gotten better. Physically. So I was like getting better and now's when I can come here.

Research Question 3: Do these perceptions vary for school age children and adolescents, and for those with a higher number of invasive procedures?

\section{School Age Children and Adolescent's Perceptions}

Matrices were created to analyze the third research question. Columns were comprised of themes with the individual codes in each theme and rows with units, number of school age and adolescent respondents per unit, and number of individual school age child and adolescent responses per unit. Analysis was conducted for each theme. Rank order from overall (PICU and GCU) total responses was Coping Strategies (504), Environmental Factors (456), Stressors (237), Procedures/Medications (198), and Information (125) with a total of 1520 responses.

Coping strategies in the PICU indicated minimal variations in percentages between age groups. The largest variation was seen in social support between school age children (22\%) and adolescents (30\%). Both age groups ranked comforting or helped first. School age children ranked behavioral distractions second and social support third. 
The reverse was seen in adolescents with social support second and behavioral distractions, third.

Rank order differences were noted between school age children and adolescents in their GCU responses. The top three GCU responses, rank ordered, for school age children were comforting or helped, social support, and behavioral distractions. Sleep in GCU was ranked first among adolescents followed by comforting or helped, and behavioral distractions. Variations between school age children and adolescents were seen in comforting or helped ( $46 \%$ vs. $21 \%$ ) as well as sleep ( $12 \%$ vs. $39 \%)$. Remaining codes showed small amount of variation between age groups. There were no responses for spirituality or trust/confidence for either age group (Table 5). 
Table 5

Coping Strategies by Age and Unit

\begin{tabular}{|c|c|c|c|c|c|c|c|}
\hline \multicolumn{8}{|c|}{ Coping Strategies (Quotes) } \\
\hline & $\begin{array}{c}\text { Comforting } \\
\text { or Helped } \\
n(\%) \\
\end{array}$ & $\begin{array}{c}\text { Social Support } \\
n(\%)\end{array}$ & $\begin{array}{c}\text { Behavioral } \\
\text { distractions } \\
n(\%) \\
\end{array}$ & $\begin{array}{l}\text { Sleep } \\
n(\%)\end{array}$ & $\begin{array}{l}\text { Spirituality } \\
\quad n(\%)\end{array}$ & $\begin{array}{c}\text { Trust/ } \\
\text { Confidence } \\
n(\%) \\
\end{array}$ & $\begin{array}{l}\text { Dreaming } \\
\quad n(\%)\end{array}$ \\
\hline PICU $(n=314)$ & 100 (32\%) & $88(27 \%)$ & 77 (25\%) & $41(13 \%)$ & $6(2 \%)$ & $2(1 \%)$ & $0(0 \%)$ \\
\hline $\begin{array}{l}\text { Number of School Age Children } \\
(n=7)\end{array}$ & $6(86 \%)$ & $7(100 \%)$ & $7(100 \%)$ & $2(28 \%)$ & $0(0 \%)$ & $1(14 \%)$ & $0(0 \%)$ \\
\hline $\begin{array}{l}\text { Number of School Age Children } \\
\text { Responses }(n=76)\end{array}$ & $27(34 \%)$ & $17(22 \%)$ & $23(29 \%)$ & $8(10 \%)$ & $0(0 \%)$ & $1(1 \%)$ & $0(0 \%)$ \\
\hline Number of Adolescents $(n=13)$ & $13(100 \%)$ & $13(100 \%)$ & $13(100 \%)$ & $10(77 \%)$ & $2(15 \%)$ & $1(7 \%)$ & $0(0 \%)$ \\
\hline $\begin{array}{l}\text { Number of Adolescent Responses } \\
(n=238)\end{array}$ & $73(31 \%)$ & $71(30 \%)$ & $54(23 \%)$ & $33(14 \%)$ & $6(2 \%)$ & $1(.1 \%)$ & $0(0 \%)$ \\
\hline GCU $(n=190)$ & $51(27 \%)$ & $38(20 \%)$ & $31(16 \%)$ & $62(33 \%)$ & $0(0 \%)$ & $0(0 \%)$ & $8(4 \%)$ \\
\hline $\begin{array}{l}\text { Number of School Age Children } \\
(n=6)\end{array}$ & $5(83 \%)$ & $2(33 \%)$ & $3(50 \%)$ & $2(33 \%)$ & $0(0 \%)$ & $0(0 \%)$ & $2(33 \%)$ \\
\hline $\begin{array}{l}\text { Number of School Age Children } \\
\text { Responses }(n=47)\end{array}$ & $21(46 \%)$ & $9(19 \%)$ & $8(17 \%)$ & $6(12 \%)$ & $0(0 \%)$ & $0(0 \%)$ & $3(6 \%)$ \\
\hline Number of Adolescents $(n=12)$ & $11(92 \%)$ & $10(83 \%)$ & $12(100 \%)$ & $11(92 \%)$ & $0(0 \%)$ & $0(0 \%)$ & $3(25 \%)$ \\
\hline $\begin{array}{l}\text { Number of Adolescent Responses } \\
n=143 \text { ) }\end{array}$ & $30(21 \%)$ & $29(21 \%)$ & $23(16 \%)$ & $56(39 \%)$ & $0(0 \%)$ & $0(0 \%)$ & $5(3 \%)$ \\
\hline
\end{tabular}


Environmental Factors ranked second with 456 overall quotes. Differences were seen in PICU for school age child and adolescent responses. The top three PICU responses by school age children, rank order, were equipment, noise, and food. Adolescent responses in PICU were hospital personnel: positive as first then equipment and change. Percentage variation was seen between age groups in hospital personnel: positive, self-determination, and equipment. A greater proportion of responses were about hospital personnel: positive and self-determination for adolescents and about equipment for school age children.

Differences were also noted for GCU between school age children and adolescent. The top three responses, rank ordered, for school age children were equipment, unit differences, and noise. Adolescents responses, rank ordered, were unit differences, noise, and equipment. Neither age group had GCU responses about change. The largest variation between groups was seen in equipment perceptions with a higher proportion of responses in adolescents (Table 6). 
Table 6

Environmental Factors by Age and Unit

\begin{tabular}{|c|c|c|c|c|c|c|c|c|}
\hline \multicolumn{9}{|c|}{ Environmental Factors (Quotes) } \\
\hline & $\begin{array}{l}\text { Equipment } \\
\quad n(\%)\end{array}$ & $\begin{array}{c}\text { Hospital } \\
\text { Personnel: } \\
\text { Positive } \\
n(\%)\end{array}$ & $\begin{array}{l}\text { Change } \\
n(\%)\end{array}$ & $\begin{array}{l}\text { Noise } \\
n(\%)\end{array}$ & $\begin{array}{c}\text { Self- } \\
\text { Determination } \\
n(\%)\end{array}$ & $\begin{array}{l}\text { Food } \\
n(\%)\end{array}$ & $\begin{array}{c}\text { Hospital } \\
\text { Personnel: } \\
\text { Negative } \\
n(\%)\end{array}$ & $\begin{array}{c}\text { Unit } \\
\text { Differences } \\
n(\%)\end{array}$ \\
\hline TOTALS $(N=456)$ & $111(24 \%)$ & $61(14 \%)$ & $43(10 \%)$ & $86(19 \%)$ & $45(10 \%)$ & $28(6 \%)$ & $7(1 \%)$ & $75(16 \%)$ \\
\hline PICU $(n=238)$ & $64(26 \%)$ & $51(21 \%)$ & $43(18 \%)$ & 31 (13\%) & $25(11 \%)$ & $16(7 \%)$ & $6(3 \%)$ & $2(1 \%)$ \\
\hline $\begin{array}{l}\text { Number of School Age Children } \\
(n=7)\end{array}$ & $7(100 \%)$ & $3(43 \%)$ & $6(86 \%)$ & $2(29 \%)$ & $1(14 \%)$ & $3(43 \%)$ & $1(14 \%)$ & $1(14 \%)$ \\
\hline $\begin{array}{l}\text { Number of School Age Children } \\
\text { Responses }(n=73)\end{array}$ & $26(36 \%)$ & $6(8 \%)$ & $15(21 \%)$ & $11(16 \%)$ & $3(4 \%)$ & $7(9 \%)$ & $4(5 \%)$ & $1(1 \%)$ \\
\hline Number of Adolescents $(n=13)$ & $10(77 \%)$ & $13(100 \%)$ & $12(92 \%)$ & $7(54 \%)$ & $7(54 \%)$ & $5(38 \%)$ & $2(15 \%)$ & $1(8 \%)$ \\
\hline $\begin{array}{l}\text { Number of Adolescent } \\
\text { Responses }(n=165)\end{array}$ & $38(23 \%)$ & $45(27 \%)$ & $28(17 \%)$ & $20(12 \%)$ & $22(14 \%)$ & $9(5 \%)$ & $2(1 \%)$ & $1(1 \%)$ \\
\hline GCU $(n=218)$ & 47 (21\%) & $10(4 \%)$ & $0(0 \%)$ & $55(26 \%)$ & $20(9 \%)$ & $12(5 \%)$ & $1(1 \%)$ & $73(33 \%)$ \\
\hline $\begin{array}{l}\text { Number of School Age Children } \\
(n=6)\end{array}$ & $4(67 \%)$ & $0(0 \%)$ & $0(0 \%)$ & $4(67 \%)$ & $1(17 \%)$ & $0(0 \%)$ & $1(17 \%)$ & $5(83 \%)$ \\
\hline $\begin{array}{l}\text { Number of School Age Children } \\
\text { Responses }(n=48)\end{array}$ & $20(42 \%)$ & $0(0 \%)$ & $0(0 \%)$ & $12(25 \%)$ & $1(2 \%)$ & $0(0 \%)$ & $1(2 \%)$ & $14(29 \%)$ \\
\hline Number of Adolescents $(n=12)$ & $8(67 \%)$ & $5(42 \%)$ & $0(0 \%)$ & $11(92 \%)$ & $7(58 \%)$ & $3(25 \%)$ & $0(0 \%)$ & $12(100 \%)$ \\
\hline $\begin{array}{l}\text { Number of Adolescent } \\
\text { Responses }(n=170)\end{array}$ & $27(16 \%)$ & $10(6 \%)$ & $0(0 \%)$ & $43(25 \%)$ & $19(11 \%)$ & $12(7 \%)$ & $0(0 \%)$ & $59(35 \%)$ \\
\hline
\end{tabular}


Stressors had a total of 237 quotes - 143 about the PICU and 94 about the GCU. For school age children, top responses about the PICU, rank ordered, were fears/scary, pain, and frustration. Adolescents' top three responses about the PICU were pain, fears/scary, and frustration. Large variations were seen between age groups with school age children having a greater proportion of responses than adolescents in fears/scary and frustration. Adolescents reported a greater proportion of responses about PICU pain than school age children.

Rank order for school age children and adolescents was consistent in the GCU: pain then fears/scary. The largest variation was noted in pain with a greater proportion of responses from adolescents (64\%) than school age children (44\%). Smaller variations were noted in time and fears with school age children providing a greater proportion of the responses than adolescents (Table 7). 
Table 7

Stressors by Age and Unit

\begin{tabular}{|c|c|c|c|c|c|}
\hline \multicolumn{6}{|c|}{ Stressors (Quotes) } \\
\hline & $\begin{array}{l}\text { Pain } \\
n(\%)\end{array}$ & $\begin{array}{c}\text { Fears/Scary } \\
n(\%)\end{array}$ & $\begin{array}{c}\text { Frustration } \\
n(\%)\end{array}$ & $\begin{array}{l}\text { Time } \\
n(\%)\end{array}$ & $\begin{array}{l}\text { Separation } \\
n(\%)\end{array}$ \\
\hline TOTALS $(N=237)$ & $119(50 \%)$ & $79(34 \%)$ & $19(8 \%)$ & $15(6 \%)$ & $5(2 \%)$ \\
\hline PICU $(n=143)$ & $64(46 \%)$ & $50(35 \%)$ & $16(12 \%)$ & $10(5 \%)$ & $3(2 \%)$ \\
\hline $\begin{array}{l}\text { Number of School Age Children } \\
(n=7)\end{array}$ & $4(57 \%)$ & $4(57 \%)$ & $2(28 \%)$ & $2(28 \%)$ & $0(0 \%)$ \\
\hline $\begin{array}{l}\text { Number of School Age Children } \\
\text { Responses }(n=51)\end{array}$ & $12(24 \%)$ & $25(49 \%)$ & $9(17 \%)$ & $5(10 \%)$ & $0(0 \%)$ \\
\hline Number of Adolescents $(n=13)$ & $10(77 \%)$ & $9(69 \%)$ & $1(8 \%)$ & $3(23 \%)$ & $1(8 \%)$ \\
\hline $\begin{array}{l}\text { Number of Adolescents } \\
\text { Responses }(n=92)\end{array}$ & $52(57 \%)$ & $25(27 \%)$ & $7(7 \%)$ & $5(6 \%)$ & $3(3 \%)$ \\
\hline GCU $(n=94)$ & 55 (58\%) & $29(31 \%)$ & $3(4 \%)$ & $5(5 \%)$ & $2(2 \%)$ \\
\hline $\begin{array}{l}\text { Number of School Age Children } \\
(n=6)\end{array}$ & $3(50 \%)$ & $4(67 \%)$ & $1(17 \%)$ & $1(17 \%)$ & $0(0 \%)$ \\
\hline $\begin{array}{l}\text { Number of School Age Children } \\
\text { Responses }(n=27)\end{array}$ & $12(44 \%)$ & $10(37 \%)$ & $1(4 \%)$ & $4(15 \%)$ & $0(0 \%)$ \\
\hline Number of Adolescents $(n=12)$ & $8(67 \%)$ & $9(75 \%)$ & $2(17 \%)$ & $1(8 \%)$ & $1(8 \%)$ \\
\hline $\begin{array}{l}\text { Number of Adolescents } \\
\text { Responses }(n=67)\end{array}$ & $43(64 \%)$ & $19(29 \%)$ & $2(3 \%)$ & $1(1 \%)$ & $2(3 \%)$ \\
\hline
\end{tabular}


Differences were seen between school age children and adolescents about Procedures/Medications in the PICU. The rank order of responses for school age children was IV, procedures and medications. Adolescent responses were in the opposite order: medications, procedures, and IV. Variations were seen between age groups across the theme. The largest variation was noted in IV with a greater proportion of responses by school age children (67\%) than adolescents (26\%).

Rank order about the GCU was consistent with the PICU rank order for school age children. Adolescents rank order changed to procedures, lab, and IV. Again, the largest variation was seen in IV with a greater proportion of school age children (65\%) responses than adolescents $(21 \%)$. Only adolescents provided responses about the lab in the GCU (Table 8). 


\section{Table 8}

\section{Procedures/Medications by Age and Unit}

\begin{tabular}{|c|c|c|c|c|}
\hline \multicolumn{5}{|c|}{ Procedures/Medication (Quotes) } \\
\hline & $\begin{array}{c}\text { IV } \\
n(\%)\end{array}$ & $\begin{array}{l}\text { Procedures } \\
n(\%)\end{array}$ & $\begin{array}{l}\text { Medications } \\
n(\%)\end{array}$ & $\begin{array}{c}\text { Lab } \\
n(\%)\end{array}$ \\
\hline TOTALS $(N=198)$ & $79(40 \%)$ & $55(28 \%)$ & $41(21 \%)$ & $22(11 \%)$ \\
\hline PICU $(n=146)$ & $58(40 \%)$ & $41(28 \%)$ & 34 (23\%) & $13(9 \%)$ \\
\hline $\begin{array}{l}\text { Number of School Age Children } \\
(n=7)\end{array}$ & $7(100 \%)$ & $2(29 \%)$ & $2(29 \%)$ & $0(0 \%)$ \\
\hline $\begin{array}{l}\text { Number of School Age Children } \\
\text { Responses }(n=49)\end{array}$ & $33(67 \%)$ & $12(24 \%)$ & $4(9 \%)$ & $0(0 \%)$ \\
\hline Number of Adolescents $(n=13)$ & $11(85 \%)$ & $10(77 \%)$ & $10(77 \%)$ & $4(31 \%)$ \\
\hline $\begin{array}{l}\text { Number of Adolescent } \\
\text { Responses }(n=97)\end{array}$ & $25(26 \%)$ & $29(30 \%)$ & $30(31 \%)$ & $13(13 \%)$ \\
\hline GCU $(n=52)$ & $21(41 \%)$ & $14(27 \%)$ & $8(14 \%)$ & $9(18 \%)$ \\
\hline $\begin{array}{l}\text { Number of School Age Children } \\
(n=6)\end{array}$ & $2(33 \%)$ & $1(17 \%)$ & $1(17 \%)$ & $0(0 \%)$ \\
\hline $\begin{array}{l}\text { Number of School Age Children } \\
\text { Responses }(n=23)\end{array}$ & $15(65 \%)$ & $5(22 \%)$ & $3(13 \%)$ & $0(0 \%)$ \\
\hline Number of Adolescents $(n=12)$ & $5(42 \%)$ & $3(25 \%)$ & $3(25 \%)$ & $3(25 \%)$ \\
\hline $\begin{array}{l}\text { Number of Adolescent } \\
\text { Responses }(n=29)\end{array}$ & $6(21 \%)$ & $9(31 \%)$ & $5(17 \%)$ & $9(31 \%)$ \\
\hline
\end{tabular}


The Information theme in the PICU demonstrated rank order differences between age groups. Thoughts and feelings were ranked first in information for both school age children and adolescents. A greater proportion of responses about advice/explanations were provided by school age children. Adolescents provided a greater proportion of the responses about knowledge. Proportion of responses about thoughts/feelings in the PICU was greater for adolescents (51\%) than school age children (42\%).

For the GCU, there were small variations in proportion of responses by school age children and adolescents. However, the rank order of the responses was the same for both age groups: advice/explanation, thoughts/feelings, and then knowledge (Table 9). 


\section{Table 9}

\section{Information by Age and Unit}

\begin{tabular}{|c|c|c|c|}
\hline \multicolumn{4}{|c|}{ Information (Quotes) } \\
\hline & $\begin{array}{c}\text { Thought and } \\
\text { Feelings } \\
n(\%)\end{array}$ & $\begin{array}{c}\text { Advice/ } \\
\text { Explanation } \\
n(\%)\end{array}$ & $\begin{array}{c}\text { Knowledge } \\
n(\%)\end{array}$ \\
\hline TOTALS $(N=125)$ & $53(42 \%)$ & $45(36 \%)$ & $27(22 \%)$ \\
\hline PICU $(n=86)$ & 42 (49\%) & $22(25.5 \%)$ & $22(25.5 \%)$ \\
\hline $\begin{array}{l}\text { Number of School Age Children } \\
(n=7)\end{array}$ & $3(43 \%)$ & $2(29 \%)$ & $2(29 \%)$ \\
\hline $\begin{array}{l}\text { Number of School Age Children } \\
\text { Responses }(n=23)\end{array}$ & $10(42 \%)$ & $7(31 \%)$ & $6(26 \%)$ \\
\hline Number of Adolescents $(n=13)$ & $10(77 \%)$ & $8(62 \%)$ & $6(46 \%)$ \\
\hline $\begin{array}{l}\text { Number of Adolescent Responses } \\
(n=63)\end{array}$ & $32(51 \%)$ & $15(24 \%)$ & $16(25 \%)$ \\
\hline GCU $(n=39)$ & $11(28 \%)$ & $23(59 \%)$ & $5(13 \%)$ \\
\hline $\begin{array}{l}\text { Number of School Age Children } \\
(n=6)\end{array}$ & $3(50 \%)$ & $2(33 \%)$ & $1(17 \%)$ \\
\hline $\begin{array}{l}\text { Number of School Age Children } \\
\text { Responses }(n=11)\end{array}$ & $3(27 \%)$ & $7(64 \%)$ & $1(9 \%)$ \\
\hline Number of Adolescents $(n=12)$ & $5(42 \%)$ & $9(75 \%)$ & $2(16 \%)$ \\
\hline $\begin{array}{l}\text { Number of Adolescent Responses } \\
(n=28)\end{array}$ & $8(29 \%)$ & $16(57 \%)$ & $4(14 \%)$ \\
\hline
\end{tabular}




\section{Invasive Procedure Scores}

Invasive procedure scores (IPS) were calculated separately for the PICU stay and the GCU stay. Cut points established for the IPS from the PICU data were also used in grouping children's IPS for the GCU, so that individual changes in groups between the PICU and GCU represent real differences in the number of invasive procedures.

In PICU, one school age child and five adolescents $(n=6)$ were in Group 1with IPS scores from 1-6. Two school age children and two adolescents $(n=4)$ were in Group 2 with IPS scores from 7-12. There were no school age children and five adolescents $(n$ $=5$ ) in Group 3 with IPS scores between 13 and 25. Four school age children and one adolescent $(n=5)$ were in Group 4 with IPS scores equal to or greater than 26.

In GCU, three school age children and seven adolescents $(n=10)$ were in IPS

Group 1. There were no school age children and one adolescent $(n=1)$ in IPS Group 2. IPS Group 3 was comprised of two school age children and four adolescents $(n=6)$. One school age child and no adolescents $(n=1)$ were in IPS Group 4.

Eight children were in the same IPS Group during their PICU stay and their GCU stay. Eight children had fewer invasive procedures in the GCU than they had in the PICU; however, 2 children experienced more invasive procedures during their GCU stay than in their PICU stay (Table 10). 


\section{Table 10}

\section{IPS Groups: PICU to GCU}

\begin{tabular}{ccccc}
\hline & \multicolumn{5}{c}{ GCU IPS Group } \\
\cline { 2 - 5 } & 1 & 2 & 3 & 4 \\
PICU IPS Group & $(\mathrm{n}=10)$ & $(\mathrm{n}=1)$ & $(\mathrm{n}=6)$ & $(\mathrm{n}=1)$ \\
\hline $1(\mathrm{n}=6)$ & 5 & 0 & 1 & 0 \\
$2(\mathrm{n}=4)$ & 3 & 0 & 1 & 0 \\
$3(\mathrm{n}=4)$ & 1 & 1 & 2 & 0 \\
$4(\mathrm{n}=4)$ & 1 & 0 & 2 & 1 \\
\hline
\end{tabular}

Two additional matrices (Tables 11 and 12) were created to display the proportions of responses in the 5 themes based on IPS groups. Rows consisted of IPS groups $1-4$, number of children responding, and total number of responses. Columns consisted of each theme.

In the PICU, a high proportion of children provided responses in each of the 5 themes, regardless of IPS group. All of the children provided responses in the Coping and Environment themes. All children in IPS groups 2 and 3 provided responses in Stressors. Across groups, all but one child (IPS group 2) provided responses about Procedures/Medications. All children in IPS groups 1, 2, and 3 as well as 3 of the 5 children $(60 \%)$ in IPS group 4 provided responses about Information. 
In the PICU, the greatest proportion of responses on Coping, Environment, and Information were from children in IPS Group 1; IPS Group 3 reported the greatest proportion of responses for Stressors. The proportions of responses for Procedures/Medications were similar in all 4 IPS groups (Table 11). In the GCU, the majority of the responses were seen in IPS Group 1 for all five themes. There were no responses for IPS Group 2 in Stressors, Procedures/Medications, and Information (Table 12). 
Table 11

\section{PICU IPS Groups and Themes}

\begin{tabular}{|c|c|c|c|c|c|}
\hline & \multicolumn{4}{|c|}{ Themes } & \multirow[b]{2}{*}{$\begin{array}{c}\text { Information } \\
(n=86)\end{array}$} \\
\hline $\begin{array}{l}\text { PICU IPS Groups } \\
(N=20)\end{array}$ & $\begin{array}{l}\text { Coping } \\
(n=314)\end{array}$ & $\begin{array}{l}\text { Environment } \\
\quad(n=238)\end{array}$ & $\begin{array}{l}\text { Stressors } \\
(n=143)\end{array}$ & $\begin{array}{c}\text { Procedures/ } \\
\text { Medications } \\
(n=146)\end{array}$ & \\
\hline \multicolumn{6}{|l|}{ IPS Group $1(n=6)$} \\
\hline Number of Children & $6(100 \%)$ & $6(100 \%)$ & $5(83 \%)$ & $6(100 \%)$ & $6(100 \%)$ \\
\hline Number of Responses $n(\%)$ & $98(31 \%)$ & $97(40 \%)$ & $28(20 \%)$ & $42(29 \%)$ & $34(40 \%)$ \\
\hline \multicolumn{6}{|l|}{ IPS Group $2(n=4)$} \\
\hline Number of Children & $4(100 \%)$ & $4(100 \%)$ & $4(100 \%)$ & $3(75 \%)$ & $4(100 \%)$ \\
\hline Number of Responses $n(\%)$ & $59(19 \%)$ & $42(18 \%)$ & $25(17 \%)$ & $29(20 \%)$ & $22(26 \%)$ \\
\hline \multicolumn{6}{|l|}{ IPS Group $3(n=5)$} \\
\hline Number of Children & $5(100 \%)$ & $5(100 \%)$ & $5(100 \%)$ & $5(100 \%)$ & $5(100 \%)$ \\
\hline Number of Responses $n(\%)$ & $87(28 \%)$ & $52(22 \%)$ & $61(43 \%)$ & $38(26 \%)$ & $15(17 \%)$ \\
\hline \multicolumn{6}{|l|}{ IPS Group $4(n=5)$} \\
\hline Number of Children & $5(100 \%)$ & $5(100 \%)$ & $4(80 \%)$ & $5(100 \%)$ & $3(60 \%)$ \\
\hline Number of Responses $n(\%)$ & $70(22 \%)$ & $47(20 \%)$ & $29(20 \%)$ & $37(25 \%)$ & $15(17 \%)$ \\
\hline
\end{tabular}


Table 12

\section{GCU IPS Groups and Themes}

\begin{tabular}{|c|c|c|c|c|c|}
\hline & \multicolumn{5}{|c|}{ Themes } \\
\hline $\begin{array}{l}\text { GCU IPS Groups } \\
(N=18)\end{array}$ & $\begin{array}{l}\text { Coping } \\
(n=190)\end{array}$ & $\begin{array}{l}\text { Environment } \\
\quad(n=218)\end{array}$ & $\begin{array}{l}\text { Stressors } \\
(n=94)\end{array}$ & $\begin{array}{l}\text { Procedures/ } \\
\text { Medications } \\
\quad(n=52)\end{array}$ & $\begin{array}{c}\text { Information } \\
\quad(n=39)\end{array}$ \\
\hline \multicolumn{6}{|l|}{ IPS Group $1(n=10)$} \\
\hline Number of children $n(\%)$ & $9(90 \%)$ & $10(100 \%)$ & $9(90 \%)$ & $7(70 \%)$ & $9(90 \%)$ \\
\hline Number of Responses $n(\%)$ & $98(52 \%)$ & $133(61 \%)$ & $54(57 \%)$ & $30(58 \%)$ & $23(59 \%)$ \\
\hline \multicolumn{6}{|l|}{ IPS Group $2(n=1)$} \\
\hline Number of Children $n$ (\%) & $1(100 \%)$ & $1(100 \%)$ & $0(0 \%)$ & $0(0 \%)$ & $0(0 \%)$ \\
\hline Number of Responses $n(\%)$ & $14(7 \%)$ & $7(3 \%)$ & $0(0 \%)$ & $0(0 \%)$ & $0(0 \%)$ \\
\hline \multicolumn{6}{|l|}{ IPS Group $3(n=6)$} \\
\hline Number of Children $n(\%)$ & $6(100 \%)$ & $5(83 \%)$ & $5(83 \%)$ & $4(67 \%)$ & $4(67 \%)$ \\
\hline Number of Responses $n(\%)$ & $58(30 \%)$ & $62(28 \%)$ & $33(35 \%)$ & $9(17 \%)$ & $9(23 \%)$ \\
\hline \multicolumn{6}{|l|}{ IPS Group $4(n=1)$} \\
\hline Number of Children $n(\%)$ & $1(100 \%)$ & $1(100 \%)$ & $1(100 \%)$ & $1(100 \%)$ & $1(100 \%)$ \\
\hline Number of Responses $n(\%)$ & $20(10 \%)$ & $16(7 \%)$ & $7(7 \%)$ & $13(25 \%)$ & $7(18 \%)$ \\
\hline
\end{tabular}




\section{CHAPTER V}

\section{DISCUSSION}

More than 200,000 children are admitted annually to PICUs in the United States (Odetola et al., 2005; Rennick et al., 2011). Many survive their critical illness physically but may have adverse psychological outcomes after discharge (Jones et al., 2006; Muranjan et al., 2008; Namachivayam et al., 2010; Shudy et al., 2006; Taylor et al., 2003). The literature describes parental concerns, stressors, and psychological outcomes (Curley, 1988; Melnyk et al., 2004; Melnyk \& Feinstein, 2001; Small \& Melnyk, 2006; Youngblut \& Brooten, 2006, 2008; Youngblut et al., 2005) yet information from the child's perspective while still in the PICU is scarce. Parents, influenced by their own experiences and emotions, typically provide information for their children (Berg-Nielson et al., 2003; De Los Reyes \& Kazdin, 2004). Research finds that proxy reports for both children and adults are not equivalent to self-report (Aastha, 2012; Alonso et al., 2010; Varni et al., 2007).

In this phenomenological study, school age children's and adolescents' perceptions of their PICU hospital experiences while in the PICU and GCU were investigated. Also examined was how their perceptions changed over time, from PICU to GCU, and whether perceptions varied for school age children and adolescents and for those with a higher number of invasive procedures. In this chapter, significant findings related to each research question, the strength and limitations of the study, implications for future research, and implications for nursing education and practice will be discussed. 


\section{Discussion of Findings}

Twenty children were interviewed in the PICU. Two children were discharged directly from the PICU. The remaining 18 children were interviewed following their transfer to the GCU. Hispanic male adolescents comprised the majority of the sample. There were 7 school age children $(8-12$ years old) and 13 adolescents $(13-18$ years old). Mean LOS was almost 3 days in the PICU and about 2 days in the GCU. More than half of the sample had had at least one GCU hospitalization, and almost half had had at least one PICU hospitalization. The average number of invasive procedures decreased from 21 in the PICU to about11 in the GCU.

RQ \#1) What are children's perceptions of their PICU hospital experiences while in the PICU and the GCU? Data from this study revealed a total of 1520 quotes from children interviewed in PICU (927) and GCU (593). Quotes were condensed until a total of 27 subthemes (codes) emerged. These substantive codes were subsumed into five overarching themes that described children's perceptions of their PICU hospital experiences. For PICU these were, in rank order, Coping Strategies, Environmental Factors, Stressors, Procedures/Medications, and Information. Environmental factors had the highest number of quotes in GCU.

Coping Strategies. Children identified the importance of social support, family and friends, frequently indicating their visits made them feel good, loved, and cared for. Several of the children stated they valued friends visiting as much or more than family visits. Friends served as a connection to their peers and the outside world. These findings are consistent with developmental expectations; Lancaster (2011) stated that adolescents may feel more comfortable sharing their thoughts and feelings with friends 
rather than parents. Conversely, some of the children indicated visitors increased their pain and anxiety. In order to rest and heal, they expressed the necessity of having a gatekeeper, usually the parents, to limit visitors during the PICU stay.

Similar to findings by Board (2005), praying and spirituality were described as effective Coping Strategies by some of the children. Two adolescents spoke of keeping their heads up, trusting in their faith and praying to help them cope with their hospitalization. One adolescent described praying a lot to keep calm and "having faith that everything would come out okay." Research investigating the effects of spirituality in hospitalized children is sparse. Zengerle-Levy (2006) found children hospitalized with severe burns felt abandoned by God, but had renewed hope when nurses provided care that incorporated spiritual support. Incorporating spirituality as part of routine nursing care for the child, not the family, is rare in the PICU environment. Asking patients about their spirituality is very different from performing a physical assessment and providing care (Ameling \& Povilinis, 2001).

Research is abundant relating to parental trust in PICU caregivers (Board \& RyanWenger, 2003; Brooten et al, 2013; Thompson, Hupcey, \& Clark, 2003; Youngblut \& Brooten, 2006, 2008; Youngblut et al. 2005); however, findings are scarce for children's need for truthfulness from parents. In this study, children spoke of betrayal in parental trust and confidence. One child, crying, expressed anger at the father's broken promise of not having to be put to sleep, only to go to surgery and be "put to sleep" a short time later. In this scary environment, surrounded by strangers, children need to be able to believe what their parents tell them. 
Environmental factors. An unexpected finding was that very few children reported disruptions in sleep from PICU noises or lighting. Studies investigating PICU noise found levels higher than acceptable (Al-Samsam \& Cullen, 2005; Carvalho et al., 2005; Milette \& Carnevale, 2003) exacerbating unfavorable environmental conditions. Most children in this study stated noises did not bother them at all, "they were used to it." This finding may be explained by children living in a large metropolitan area attending large schools. One adolescent stated he had lots of siblings and was used to the noise. One adolescent identified nurses' talking outside the room and the light from outside the room as interrupting sleep. For another adolescent, the room lights increased the pain and interfered with sleep. Visitors' talking also contributed to sleep loss by increasing children's pain levels. Some children described sleep disruptions from aerosol treatments, medication administration, or vital signs, especially the automatic noninvasive BP monitor. Interestingly, the children were told the BP cuff was being left on so as not to disturb their sleep.

Coyne (2006) found a loss of self-determination with children hospitalized on the GCU. Results from the current study of children in the PICU also found a loss of selfdetermination, particularly with privacy and control. Children of both age groups in the PICU described having to urinate with curtains open and being uncovered perceiving full genitalia exposure. They required permission to get in or out of bed and were not allowed to leave the room. Children described being strapped down from monitors or IVs. This lack of privacy and control, typically so vital to adolescents, was described by both age groups. 
Children wished the PICU room was a brighter, more colorful environment. One child described painting the walls pink, making the floors shiny, and putting flowers on the ceiling. Many of the interviews contained descriptions of the PICU window as looking only at other buildings, rocks, or other windows. Some children reported not knowing what the weather was like outside or whether it was day or night. The importance of a window that provides natural lighting and a view of the outside was conveyed in many of the children's perceptions.

Stressors. Two adolescents described anxiety and fear exhibited by their mothers. Similar to children after the death of a sibling in the PICU/NICU (Youngblut \& Brooten, 2014), adolescents became reluctant to discuss their own anxiety, fears, and concerns for fear of increasing their parents' stress. A school age child had trouble sleeping because of heightened family anxiety; family members woke the child frequently to ask how the child was feeling. In their study with 2- to 7-year-old children, Small and Melnyk (2006) found early increases in maternal state anxiety were significantly related to higher levels of children's post-discharge after a PICU stay externalizing behaviors such as hyperactivity and aggression. Melnyk and Feinstein (2001) found that high maternal anxiety and high child anxiety frequently were linked. Studies were not found relating to adolescent outcomes.

Procedures/Medications. Consistent with previous research of hospitalized children (Coyne, 2006), many children in the PICU discussed procedures as disturbing and fearful. Similar to findings by Rennick and colleagues (2002), many of these procedures may be viewed as minor and non-invasive by health care personnel; however, children's perceptions differed. As in other research (Blount et al., 2009; Coyne, 2006), 
the children had anticipatory distress, particularly regarding needles and IVs. They described frustration and fear relating to the pain or frequency of these invasive procedures. One child described intense fear of the "doctors and what they had in their pockets" whenever they came near the room, expecting a "stick" from them, although the child was not anticipating another "shot" other than the first one. Consistent with findings with hospitalized children (Carney et al., 2003; Koening et al., 2003; Lindke et al., 2006; Salmela et al., 2009; Wennström \& Bergh, 2008), children in both age groups reported fear of pain (surgical and needles) or procedures.

Information. Children were asked what advice and expectations they would offer friends if they had to be hospitalized in PICU. This question provided rich insight into children's perceptions. Many said they would tell their friends to not be afraid; people were there to help them. The need for social support through friends visiting in the PICU was apparent in many interviews. Children also would advise others to expect "large, overwhelming" machines as well as the scary name of the unit itself, "PICU." Children's advice and expectations for their friends have not been reported in other research of children's perceptions of hospitalization.

Overall quotes in GCU were less in frequency and indicated perceptions consistent with the PICU interviews. More responses were recorded for Environmental Factors in GCU and for Coping Strategies in PICU. Consistent with other findings (Board, 2005; Karande et al., 2005; Muranjan et al., 2008; Playfor et al., 2000), many children in this study were able to recall positive and negative aspects of their PICU stay during their GCU interview. 
Environmental Factors. Children interviewed in GCU recalled monitors attached to them, but many did not understand their purpose. They now addressed how annoying they were, particularly the automated BP cuff. Children were now able to recognize the freedom they felt from no longer being attached to monitors. They described noises not mentioned in the PICU interview, such as the flushing of the toilet in the bathroom shared between PICU rooms. They identified not being bothered by monitors or IV alarms but by nurses talking when they were trying to sleep. Coyne also (2006) found hospitalized children were disturbed by nurses talking, bright lights, and babies crying. Children in her study identified being able to sleep through noises as they adapted to hospital routines.

Two children described the lack of privacy in the PICU interview and addressed it again in the GCU interview. They spoke of the privacy they were afforded in the GCU and one adolescent understood his difficulty in urination in the PICU was not from a health condition but from the lack of privacy.

In this study, children frequently used food as a behavioral distraction, eating from boredom, consistent with Board's results (2005). Children said they liked the food and being able to order it themselves in both interviews.

Children in PICU interviews described the inability to see outside and the lack of sunshine. Many of the children described playing outside when they were home. Responses in GCU reflected recognition of vast differences in room appearance with the GCU being prettier. The GCU windows offered an opportunity to see sunshine, birds, and people and to know they would be outside soon. Most children remarked about the 
differences in the view as they were able to see outside. They understood that transfer to the GCU indicated they were not as sick as in the PICU and were closer to going home.

Coping Strategies. Responses in this theme in the GCU were consistent with the PICU interview. Children described parents, friends, and nurses in the PICU providing comfort, help, and social support. Adolescents remembered friends providing a different type of distraction and social support; they wanted to enjoy every moment their friends visited. Unlike the PICU interview, a school age child and an adolescent described in the GCU interview cherished items brought from home as providing comfort. Board (2005) and Ryan-Wenger and Gardner (2010) found various Coping Strategies in their interviews with children; however, the importance of friends visiting and personal items from home were not identified. Contrary to other research, some children perceived their visitors as increasing pain levels while they were in PICU.

Consistent with other PICU studies (Board, 2005; Ryan-Wenger \& Gardner, 2010), children recalled watching, TV, sleeping, and eating as behavioral distractions. Adolescents recalled using their cell phones as a distraction which has not been reported in other studies. One adolescent described embarrassment when he rolled onto his nurse call button, disturbing the nurse. Adolescents felt awkward, insecure, and very easily embarrassed, which Pickhardt (2013) attributes to lower self-esteem.

Stressors. Children in PICU interviews recalled pain specific to their diagnoses, procedures, and IVs (medications and infiltration). An adolescent described the severe pain he experienced when his IV was not working properly. Children discussed pain management; some found it to work well, while others reported inadequate relief. Studies have addressed the pain experienced with invasive procedures such as IVs 
(Rennick et al., 2002); however, children in this study discussed other causes of IV pain and the effectiveness of a topical spray to decrease pain with finger sticks.

Children recalled fear of shots and needles and described still being afraid of them in the GCU interview. Children on GCU, but not in PICU, described fear of radiology reports and their illness causing them to miss school and consequently being separated from their peers. One adolescent in GCU described missing his pets and his bed.

Procedures/Medications. Children continued to identify medications and specific treatments (e.g. respiratory aerosols) helping them to improve. They described frustration with repeated lab work; however, responses on GCU indicated an increased tolerance with more sticks and waiting for results. They verbalized understanding that they needed these to be able to go home.

Information. Consistent with the PICU interview, one adolescent in the GCU interview identified a frustration with the PICU admission after he required a transfer from the rehabilitation unit. A second adolescent expressed anger related to his readmission to PICU. Both children felt their initial hospitalizations were futile. Adolescents in PICU who indicated the name "PICU" was scary realized by their GCU interview that it just meant they were going to receive special care. This finding has not been reported in other studies. Children also would advise others to bring things from home to keep them busy or provide comfort, such as a special stuffed bear or pillow.

RQ \#2) How do children's perceptions of their PICU experiences change over time; PICU to GCU? Children's responses decreased from 927 in the PICU interviews to 593 in the GCU interviews; however, children recalled aspects of the PICU stay during the GCU interview. New responses were seen during the GCU interview with children 
expounding in some of the themes. The initial PICU interview was met with trepidation by both children and parents. After consenting, some parents were reluctant to leave the room and some children were somewhat reticent to talk. However, after that, families and children frequently were proactive in arranging times for the second interview. Children may have viewed the GCU interview as beneficial, presenting an opportunity to verbalize their perceptions of the PICU hospitalization. Children now knew what to expect in the interviews. Both children and their parents verbalized their desire to help, to give information in an effort to improve outcomes for children subsequently admitted to PICU. Some of the parents and children asked, "Did I say things that will help?" Rank order of themes changed between PICU and GCU with Coping Strategies having the largest number of responses in PICU (314) and Environmental Factors (218) in GCU. The remaining themes remained unchanged in rank order.

Coping Strategies. The proportion of responses regarding Coping Strategies in PICU (33.9\%) was similar to the proportion in GCU (32\%). A larger percentage of responses was seen in comforting or helped in PICU (31.8\%) compared to GCU (26.9\%). More responses were seen relating to sleep in GCU (32.6\%) compared to PICU (13.1\%). There were no responses about dreaming in PICU, but in GCU, 8 children recalled dreams they experienced in PICU. Responses were seen in spirituality (1.9\%) with children describing having faith and trust/confidence and using prayer in PICU but not in GCU.

Environmental Factors. Environmental Factors ranked second in number of PICU responses (25.7\%) and first in GCU responses (36.7\%). The responses in GCU were more positive than those in the PICU. Children in GCU were happy about being 
able to shower in individual private bathrooms and to look out the windows. They described freedom from IVs and monitors, or "computers" as they were frequently called. They were no longer confined to their beds or rooms. Responses about Equipment were greater in PICU (27\%) than in GCU (22\%). Children in PICU described the overwhelming feelings they had with the amount of IV pumps and various monitors. Almost twice as many responses in GCU (25.2\%) compared to PICU (13\%) were about noise, contrasting the quiet of the GCU with the many sounds in the PICU.

Stressors. Stressors ranked third for both PICU (15\%) and GCU (16\%) with very similar percentage of responses. Pain had the largest number of responses in PICU (45\%) and a larger percentage in GCU (59\%). The second largest amount of responses was seen in fears/scary for both interviews, 35\% in PICU (35\%) and 31\% in GCU.

Procedures/Medications. Procedures/Medications ranked fourth for both units with $16 \%$ in PICU and $9 \%$ in GCU. The proportions of responses about IVs ( $40 \%$ in both units) and Procedures (28\% PICU and 27\% GCU) were almost equal across units.

Information. Lastly, PICU comprised 9\% of responses in Information compared to 7\% in GCU. Responses about thoughts and feelings were more common in PICU (49\%) than in GCU (29\%); the proportion of responses about advice/expectations in GCU (59\%) was more than double that in PICU (26\%).

Research on children's memories following an intensive care stay found an amnesic effect from the opioids and benzodiazepines (Playfor et al., 2000). Colville et al. (2008) found delusional memory in children was significantly associated with longer hospital stays and longer duration of opiates and benzodiazepines. Children receiving these drugs were nearly five times more likely to report delusional memory than those not 
receiving those medications. Children reported delusional memories after receiving these medications for less than two days. In this study, only one adolescent received a benzodiazepine as needed, not as a continuous infusion, which may account for the lack of delusional thoughts and memories observed in this sample.

RQ \#3) Do these perceptions vary for school age children and adolescents, and for those with a higher number of invasive procedures? Frequency of perceptions varied slightly across themes, affecting the rank order, for school age children and adolescents. Contrary to results by Lindeke et al. (2006) suggesting children's responses varied by developmental ages, this study found similar levels and types of responses by school age children and adolescents. Both school age children and adolescents verbalized understanding people were there to help them and to not be afraid, and made valuable suggestions for improving hospitalization. There was a smaller participation for school age children $(n=7)$ compared to adolescents $(n=13)$ in PICU with a smaller number of overall responses for school age children compared to adolescents. Smaller differences in proportion of responses was found between age groups on GCU compared to PICU which may be explained by increased comfort levels with hospital setting, smaller time interval between interviews, and anticipation of discharge. Children, although cooperative, may have more opportunity for distraction and less interest in interviewing.

Coping Strategies. Proportion of response in PICU for Coping Strategies was similar between age groups for all codes. There were no responses for school age children in spirituality unlike adolescents who were more comfortable in describing their beliefs and faith. The largest percent of responses for school age children and adolescents was in comforting or helped with responses including the presence of parents 
and nurses, particularly those using humor. The rank order of behavioral distractions and social support for school age children (second and third, respectively) was opposite that for adolescents (third and second, respectively).

Consistent with developmental expectations (Broderick \& Blewitt, 2009), school age children identified the use of TV or video games as a behavioral distraction while adolescents enjoyed visiting with their friends and using cell phones for social communication. Adolescents, more than school age children, placed a higher value on visits by peers than by family. Perhaps this indicates their struggle for increasing independence from parents. Adolescents talked about visits from their friends as being happier, perhaps because of the difference in the type of relationships adolescents have with their parents and their friends. Studies by Board (2005) and Ryan-Wenger and Gardner (2012) found children valued visiting and support of families and nurses as peers were not available during hospitalization. Results from this study found children greatly valued maintaining contact with their friends through visitation and/or cell phones.

Coping Strategies that adolescents used, such as napping, may preserve some feeling of control. Board (2005) found sleeping or taking a nap to be the most frequently reported coping strategy, with children napping to maintain a sense of control. RyanWenger (1992) described sleeping as a behavioral avoidance activity children used to avoid stressors other than isolation. Children in the current study stated they napped from boredom; however, the previous explanations may have held true as well. Adolescents appreciated nurses who took time to listen to them, treated them as individuals, and demonstrated humor; jokes frequently relieved anxiety (Pelander et al, 
2006) indicating that interactions with healthcare professionals may have a direct impact on children's coping.

Children in both age groups indicated they enjoyed playing x-box. Research has not mentioned the use of video games during PICU hospitalization. Griffiths (2005) found video games provided cognitive distraction for children during chemotherapy and may be useful as an adjuvant for pain management. A randomized controlled trial by Kato, Cole, Bradlyn, and Pollock (2008) found the use of video games improved medication adherence, self-efficacy, and knowledge in patients 13-29 years old with cancer.

Not mentioned in other studies was the importance of maintaining social support through social media. Unlike school age children, the majority of the adolescents reported texting their friends, sending pictures through iCHAT, and keeping in contact through Facebook. One adolescent validated the need for social connection by expressing his extreme frustration with having no internet service while in PICU. These findings may indicate that adolescents are placing a greater emphasis on social rather than family relationships (Broderick \& Blewitt, 2009).

Environmental Factors. In PICU interviews, school age children had the highest number of responses for equipment; adolescents was hospital personnel: positive. Developmentally, school age children were most likely unable to understand the purpose and sounds of the equipment. Consistent with Ryan-Wenger and Gardner (2012), adolescents enjoyed nurses who told jokes and had a good sense of humor.

Although not ranked high in PICU or GCU, self-determination was seen in both age groups as they lost the ability to decide some of the most basic aspects of their lives - 
when they eat, sleep, and use the bathroom. Many of them felt restrained from IVs and monitors. Some described tubes as preventing them from turning. This loss of control was voiced by school age children and adolescents. Wennström et al. (2009) described school age children (6 - 9 years) trying to gain control during hospitalization. Coyne (2006) found a similar theme, being in an unfamiliar environment, in her study of children's GCU perceptions. Feelings of frustration and powerlessness resulted from having to conform to hospital ward routines (Coyne, 2006). The child's loss of selfdetermination was exacerbated by hospital fears and anxieties.

Stressors. School age children in PICU had a higher number of responses in "fears/scary" followed by pain and the ranks were reversed for adolescents, pain first then fears/scary. Frustration was ranked third in both age groups. Rank order differences between age groups is consistent with developmental expectations. School age children are learning to tame their imagination and adolescents are struggling with their sense of control and identity (Broderick \& Blewitt, 2009).

Procedures/Medications. School age children had more responses about IVs (67\%) and procedures (29\%) in PICU; adolescents had more with medications (31\%) followed by procedures (30\%). Children reported perceptions of pain with both IVs and procedures. Adolescents, because of their advanced cognitive level, were able to discuss types and purposes of medications more than school age children. Consistent with other studies, hospitalized school age children have reported fears related to nursing procedures (Salmela et al., 2009), strange environments, equipment, pain (Wennström et al., 2008; Salmela et al., 2009), lack of privacy and discretion, restricted free will, rejection, bodily injury (Wennström \& Bergh, 2008), and injections and needles (Salmela et al., 2009). 
Lack of information, surgery, and disease symptoms also have been identified as fear provoking (Wennström \& Bergh, 2008; Salmela et al., 2009). Research indicates the most common fears for children of all ages are related to pain and nursing procedures (Carney et al., 2003; Lindke et al., 2006; Salmela et al., 2009).

Information. Thoughts and feelings had the highest number of PICU responses for both school age children and adolescents. This finding is not surprising as children described their reason for PICU hospitalization, fears with the uncertainty of the event, and frustration relating to missing summer and first jobs. The second highest number of responses was in advice and expectations for school age children and knowledge for adolescents. Developmentally, school age children want to be helpful; giving advice would serve to fill that need. The larger response rate for adolescents in information may be related to their more mature cognitive levels and previously hospitalizations.

The Invasive Procedure Score (IPS) checklist (Rennick et al., 2002) was used to measure the numbers and types of invasive procedures (tissue damaging or non-tissue damaging) experienced by each child, as recorded in the child's medical record. Four groups were created using the score quartiles for this sample as cut points; IPS Group 1 had the fewest procedures (first quartile) and IPS Group 4 had the most (fourth quartile).

Results from the current study indicated that most children had the same number or fewer invasive procedures in the GCU as they had in the PICU. Two children experienced more invasive procedures during their GCU stay than their PICU stay. This may be due in part to the difference in blood drawing in the two units. In the PICU, children usually have lines from which blood can be drawn, avoiding an invasive procedure; whereas, blood is usually drawn through venipuncture in the GCU. They also 
experienced radiology procedures and painful physical therapy. Children recovering in GCU experiencing more invasive procedures than PICU suggest outcomes may not be affected by PICU IPS scores alone.

All children in the PICU in all IPS groups provide responses in the Coping and Environment themes. In the PICU, IPS Group 1 provided the greatest proportion of responses on Coping, Environment, and Information. All children provided responses in Coping Strategies and Environmental Factors, they all described various coping strategies used to help them through their PICU hospitalization. All children in IPS Groups 2 and 3 provided response in Stressors; however, IPS Group 3 reported the greatest proportion of responses for Stressors. The latter may be explained by children who experienced higher numbers of invasive procedures describing more pain and fears. The majority of responses for children in the GCU was seen in IPS Group 1 for all five themes.

Rennick and colleagues $(2002,2004)$ conducted extensive research comparing psychological responses of children hospitalized in PICU with those hospitalized in a GCU to identify clinically relevant factors associated with their psychological outcomes. Results indicated children who were younger, more severely ill, and who experienced more invasive procedures had significantly more medical fears, lower sense of control over their health, and ongoing psychological distress 6 months post discharge. They found length of stay and severity of illness in the younger child, coupled with invasive procedures may have adverse long-term effects, regardless of hospital unit.

\section{Significance}

Five studies of children's PICU perceptions were based on children's responses, not solely on parent report (Barnes, 1975; Board, 2005; Cataldo, Bessman, Parker, 
Pearson, \& Rogers, 1979; Rennick et al., 2002; Ryan-Wenger \& Gardner, 2012). Only the studies by Board (2005) and Ryan-Wenger and Gardner (2012) were conducted while children were still in the hospital but following PICU discharge. This study is the first study eliciting children's perceptions of PICU hospitalization from the children and while they were still in the PICU. A second interview following their transfer to GCU, although still in the hospital, provided the opportunity to investigate how perceptions changed over time. Results provided valuable insight into the child's view as told from their perspective.

Although results were similar to some previously identified in the literature, there were several findings not reported in prior research in hospitalized children. These included: the importance of honesty when parents are communicating with children; the importance of peer visitation; children's substantial reliance on social media and texting to maintain contact with peers; equipment, such as IVs, being viewed as overwhelming due to their size and/or number; the lack of understanding/knowledge related to equipment; and the name PICU itself presenting a scary, overwhelming connotation to children.

It is important that health care personnel recognize children's and the various coping strategies they use to deal with the hospitalization experience as it is happening. There has been an increase in research relating to children's hospitalization, much of it focusing on the experience of the parents with relatively little being told from the child's perspective. Rennick et al. (2002) has suggested there is a marked potential for ongoing psychological disturbance following hospitalization. 
These findings are significant for children, families, health care providers, and hospital administration as they identify specific perceptions children have relating to their PICU hospitalization. This study sheds light on differences in perceptions between school age children and adolescents at two different time points and those with higher number of procedures. Children with more severe disease and those left with ongoing deficits may experience additional stressors following discharge. It is important to understand their hospitalization perceptions to form a plan of care to mitigate adverse experiences as they happen. This plan of care may improve children's immediate outcomes as well as those post-discharge. Identifying adverse factors and providing relevant interventions would help to decrease length of stay and minimize readmissions resulting in cost savings for families, organizations, and insurance companies.

\section{Limitations of the Study}

Research findings differ on the best interview techniques for children. This study was designed to use an open-ended, conversational-style interviewing technique with a semi-structured interview guide followed by probes to elicit more specific information. Many of the children were extremely ill and tired easily when answering questions, especially in the PICU. Some were not able to communicate well, using grunts, noises, or gestures to completely express their thoughts. Two children had an extremely difficult time communicating due to severe neurologic involvement, struggling to say everything they wanted conveyed. As a result, interviews occasionally became more closed-ended with children answering yes, no, or using non-verbal answers, such as pointing. Nonverbal responses were not analyzed with the audio recordings creating a study limitation. 
The participants in this study were primarily male, Hispanic, and adolescents. Although consistent with county and hospital admission demographics, having more female, school-aged, and non-Hispanic children in the sample may have led to different findings.

Another limitation was time constraints during the GCU interview. Many of the children were told they were going home or had received their discharge paperwork. Anticipating their imminent discharge may have resulted in children rushing through the interview in order to leave the hospital sooner.

These children had relatively short PICU length of stays creating an additional limitation. Including children with longer lengths of stay may provide a larger number of responses and additional information.

Lastly, a large limitation was the lack of children in the sample who required mechanical ventilation with sedative administration. A sample of more critically ill children may provide different data. Sedatives, particularly benzodiazepines, have been found to have an amnesic effect possibly altering the child's memory of certain PICU events.

\section{Implications for Future Research}

Findings from this study provide many implications for future research. Children were interviewed while in PICU with a second interview occurring following transfer to the GCU. Further research is needed to investigate memory of the child's PICU experience following discharge. A longitudinal study including measures of stress, psychological well-being, depression, anxiety or post-traumatic stress disorder (PTSD) could provide valuable information on the part that these variables may play in children's 
memories and outcomes. Additionally, including more children who had received mechanical ventilation and opiate administration in the sample may provide a broader array of responses.

Research on the feasibility and effects of using social media technology by children in the PICU is needed. These results indicated children wanted to maintain contact with their peers which were accomplished through their cell phones. A study comparing children in the PICU who use social media with those not using social media would yield broader knowledge of differences this technology can have.

There is a paucity of data on the effect of natural light and noise on outcomes of critically ill children. The many studies of infants in the NICU may not apply to the children in the PICU because of differences in environment, children's age and development, and use of different types of equipment. Several of the children identified disturbances in their sleep as a result of visitors or bright light, either from within or outside the room. Although the majority of the children stated noise did not bother them, findings relating to basic interventions to decrease noise, improve lighting, and improve sleep could serve to improve not only the child's perceptions but pain control.

Another potential area of research is regarding the provision of age-appropriate PICU educational information (e.g. booklets) on children's perceptions and outcomes relating to their sense of empowerment and anxiety. Many children in this study indicated a lack of knowledge regarding equipment, noises, treatment, and plan of care. Seeking information is a common strategy among school age children for coping with the stressful aspects of hospitalization (Coyne, 2006; Pelander, 2005). Proactively educating may help to improve their perceptions. 


\section{Implications for Nursing Education and Practice}

The results of this research are important for nursing education and nursing practice. Disseminating children's PICU perceptions would help nurses and the health care team to understand developmental expectations and to improve care delivery and child outcomes, both short and long term. Health care personnel may need to be reminded of the importance of privacy to children. Interdisciplinary education on simple strategies that are in the control of healthcare personnel, such as securely closing curtains, would improve children's perceptions and ultimately psychological outcomes. Both children recounted their lack of privacy during the PICU and GCU interviews. Minimizing pain through the use of topical anesthetics and improved pain management needs to be re-emphasized.

Environmental factors should be considered in the design and building of new hospitals. The results of this study provide examples of ways to make PICUs into more child friendly units. Children need to see outside and natural light. Perhaps changing the type of lighting or placing murals or films with continuous feeds and on the windows that provide no "view" could compensate for the inability to see outside.

Education needs to include sources of noise and ways to mitigate it. Studies have examined the effects of alarm fatigue on safety and nurses; the beeping disturbs the patients as well.

Some children had negative experiences with larger numbers of visitors, but also expressed the need to see their friends. Changes in the definition of family and addressing hospital visitation have led to relaxed visiting policies in many organizations. Patientand family-centered care has led to open visitation with minimal restrictions on amount 
of visitors allowed, even in the PICU. Consequently, multiple visitors may congregate in one PICU room at any given time in an effort to provide family respite and diversion. Decisions relating to visitors should be a collaborative decision between families, members of the health care team, and most importantly the child (Lee et al., 2007).

\section{Summary}

The purpose of this study was to investigate children's perceptions of their PICU hospitalization. Hospitalization can be a formative experience for children as it may threaten their sense of independence and security. This study provided valuable insight into the children's perceptions of their PICU hospitalization as told from their voices.

Results of this study indicated children desire open, honest communication. They emphasized the importance of peer support and visitation, especially adolescents.

Adolescents strongly relied on social media and texting through cellular phones to remain in contact with friends. Children verbalized fear of shots (IVs), frustration with having repeated lab work, feeling overwhelmed by the number and size of various PICU equipment, and the name "PICU" presents a scary, overwhelming connotation.

In summary, admission to the PICU is a very stressful event for children. Results found new information not previously found in the literature. Incorporating the findings from this study may suggest ways to ameliorate adverse effects for children from their PICU hospitalization. Further studies regarding effects of PICU hospitalization and critical illness on children are necessary to identify ways to moderate children's perceptions and improve psychological outcomes. 


\section{REFERENCES}

Aastha, B. (2012). Reliability of PedsQL Neuromuscular module in assessing healthrelated quality of life in children with Down syndrome. International Journal of Therapies and Rehabilitation Research, 1(1), 6 - 11. doi: 10.5455/ijtrr.00000007

Akansel, N., \& Karmakci, S. (2008). Effects of intensive care unit noise on patients: A study on coronary artery bypass grafts. Journal of Clinical Nursing, 17(12), 1581- 1590. doi: 10.1111/j.1365-2702.2007.02144.x. Aldridge,

Aley, K. E. (2002) Developmental approach to pediatric transplantation. Progress in Transplantation, 12(2)2, 86-91.

Alonso, E. M., Limbers, C. A., Neighbors, K., Martz, K., Bucuvalas, J. C., Webb, T., \& Varni, J. W. (2010). Cross-sectional analysis of health related quality of life in pediatric liver transplant recipients. Journal of Pediatrics, 156(2), 270- 276. doi:10.1016/j.jpeds.2009.08.048

Al-Samsam, R.H., \& Cullen, P. (2005). Sleep and adverse environmental factors in sedated mechanically ventilated pediatric intensive care patients. Pediatric Critical Care Medicine 6(5):562-567.

Ameling, A. \& Povilions, M. (2001). Spirituality, Meaning, Mental Health and Nursing. Journal of Psychosocial Nursing and Mental Health, 39(4) 14-20.

American Academy of Pediatrics. (2012). Policy Statement: AAP Publications Reaffirmed. Pediatrics, 129(2), e561. doi:10.1542/peds.2011-3210

American Academy of Pediatrics. (2006). AAP. Publications retired and reaffirmed. Pediatrics, 117(5), 1846-1847. doi: 10.1542/peds.2006-0676

American Academy of Pediatrics, \& American College of Obstetricians and Gynecologists. (2007). Guidelines for perinatal care (6th ed.). Elk Grove Village, IL: American Academy of Pediatrics.

American Academy of Pediatrics, Committee on Environmental Health. (1997). Noise: A hazard for the fetus and newborn. Pediatrics, 100(4), $724-727$. doi:: 10.1542/peds.100.4.724

Bailey, E., \& Timmons, S. (2005). Noise levels in PICU: An evaluative study. Pediatric Nursing, 17(10), 22-26. 
Balluffi, A., Kassam-Adams, N., Kazak, A., Tucker, M., Dominguez, T., \& Helfaer, M. (2004). Traumatic stress in parents of children admitted to the pediatric intensive care unit. Pediatric Critical Care Medicine, 5(6), 547 - 553.

doi: 10.1097/01.PCC.0000137354.19807.44

Bares, C. B., \& Gelman, S. A. (2008). Knowledge of illness during childhood: Making distinctions between cancer and colds. International Journal of Behavioral Development, 32(5), 443-450. doi: 10.1177/0165025408093663

Barnes, C. M. (1975). Levels of consciousness indicated by responses of children to phenomena in the Intensive Care Unit. Maternal-Child Nursing, 4(4), 215-290.

Barnes, C. M., Kenny, F. M., Call, T., \& Reinhart, J. B. (1972). Measurement in management of anxiety in children for open heart surgery. Pediatrics, 49(2), 250-259.

Barry, T. D., Dunlap, S. T., Cotton, S. J., Lochman, J. E., \& Wells, K. C. (2005). The influence of maternal stress and distress on disruptive behavior problems in boys. Journal of American Academy of Child and Adolescent Psychiatry, 44(3), 265 - 273. doi: 10.1097/00004583-200503000-00011

Bellack, J. P., \& Fleming, J. W. (1996). The use of projective techniques in pediatric nursing reserach. Journal of Pediatric Reserach, 11(1), 10-28.

Berg-Nielsen, T. S., Vika, A., \& Dahl, A. A. (2003). When adolescents disagree with their mothers: CBCL-YSR discrepancies related to maternal depression and adolescent self-esteem. Child: Care, health, and development, 29(3), 207-213. doi: 10.1046/j.1365-2214.2003.00332.x

Berglund, B., Lindvall, T., Schwela, D. H. (1999). Guidelines for Community Noise. Geneva: World Health Organization. Retrieved from http://www.who.int/docstore/peh/noise/Comnoise-1.pdf

Bering, J., \& Bjorklund, D. F. (2004). The natural emergence of reasoning about the afterlife as a developmental regularity. Developmental Psychology, 40(2), 217-233.

Berwick, D., \& Kotagal, M. (2004). Restricted visiting hours in ICUs: Time to change. Journal of the American Medical Association, 292(6), 736-737.

Bibace, R., \& Walsh, M. E. (1980). Development of children's concepts of illness. Pediatrics, 66(6), 912-917. 
Blount, R. L., Zempsky, W. T., Jaaniste, T., Evans, S., Cohen, L. L., Devine, K. A., \& Zeltzer, L. K. (2009). Management of pediatric pain and distress due to medical procedures $\left(4^{\text {th }}\right.$ ed.). Handbook of Pediatric Psychology (pp. $\left.171-188\right)$. New York, NY: Guilford.

Board, R. (2004). Father stress during a child's critical care hospitalization. Journal of Pediatric Health Care, 8(5), 244-249. doi:10.1016/j.pedhc.2004.06.003

Board, R. (2005). School-age children's perceptions of their PICU hospitalization. Pediatric Nursing, 31(3), 166-175.

Board, R., \& Ryan-Wenger, N. (2000). State of the science on parental stress and family functioning in pediatric intensive care units. American Journal of Critical Care, 9(2), 106-122.

Board, R., \& Ryan-Wenger, N. (2003). Stressors and stress symptoms of mothers with children in the PICU. Journal of Pediatric Nursing, 18(3), 195- 202. doi: 10.1053/jpdn.2003.38

Breitbart, W., Marotta, R., Platt, M.M., Weisman, H., Derevenco, M., Grau, C.,... Jacobsen, P. (1996). Clinical update: Double-blind trial of Haloperidol, Chlorpromazine, and Lorazepam in the treatment of delirium in hospitalized AIDS patients. American Journal of Psychiatry, 153, 231-237. doi: 10.1002/(SICI)1099-1611(199703)6:1<75::AID-PON259>3.0.CO;2-R

Broderick, P. C., \& Blewitt, P. (2010). The emerging self and socialization in the early years. The life span: Human development for helping professionals $\left(3^{\mathrm{rd}} \mathrm{ed}\right.$., $\mathrm{pp}$. 9-10). Upper Saddle River, NJ: Pearson Education Inc.

Bronner, M. B., Knoester, H., Bos, A. P., Last, B. F., \& Grootenhuis, M. A. (2008). Posttraumatic stress disorder (PTSD) in children after pediatric intensive care treatment compared to children who survived a major fire disaster. Child and Adolescent Psychiatry and Mental Health, 2(9), 966-974. doi:10.1186/1753-2000-2-9

Brooten, D., Youngblut, J. M., Seagrave, L., Caicedo, C., Hawthorne, D., Hidalgo, I., \& Roche, R. (2013). Parent's perceptions of health care provider actions around child ICU death: what helped, what did not? American Journal of Hospice and Palliative Medicine, 30(1), 40-49. doi: 10.1177/10499091124430

Callender, K. A., Olson, S. L., Choe, D. E., \& Sameroff, A. J. (2012). The effects of parental depressive symptoms, appraisals, and physical punishment on later child externalizing behavior. Journal of Abnormal Child Psychology, 40(3), 471-483. doi: 10.1007/s10802-011-9572-9 
Carney T., Bishop E., Kerr C., McClure J., Murphy S., Parker J., Scott, F., Shields C., \& Wilson, L. (2003) Children's views of hospitalization: an exploratory study of data collection. Journal of Child Health Care, 7, 27-40.

Carno, M. A., \& Connolly, H. V. (2005). Sleep and sedation in the pediatric intensive care unit. Critical Care Nursing Clinics of North America, 17(3), 239-244.

Carno, M. A., Hoffman, L. A., Henker, R., Carcillo, J., \& Sanders, M. H. (2004). Sleep monitoring in children during neuromuscular blockade in the pediatric intensive care unit: A pilot study. Pediatric Critical Care Medicine, 5(3), 224-229. doi: 10.1097/01.PCC.0000124024.92280.F9

Carvalho,W. B., Pedreira, M. L. G., \& deAguiar, M. A. L.(2005). Noise level in a pediatric intensive care unit. Journal of Pediatrics, 81(6), 495-498.

Caspi, A., Harrington, H., Milne, B., Arnell, J. W., Theodore, R. F., \& Moffitt, T. E. (2003). Children's behavioral styles at age 3 are linked to their adult personality traits at age 26. Journal of Personality, 71(4), 495 - 514. doi: 10.1111/1467-6494.7104001

Cataldo, M.F., Bessman, C.A., Parker, L.H., Pearson, J.E., \& Rogers, M.C. (1979). Behavioral assessment for pediatric intensive care units. Journal of Applied Behavior Analysis,12(1), 83-97. doi.org/10.1901

Centers for Disease Control and Prevention, National Center for Health Statistics, Health Data Interactive (2010). Persons with hospital stays in the past year, by selected characteristics Health, United States, 2010, table 98. Retrieved from http://www.cdc.gov/nchs/data/hus/hus10.pdf\#098

Cmeil, A. C., Karr, D.M., Gasser, D. M., Oliphant, L. M., \& Neveau, A. J. (2004) Nosie control: A nursing team's approach to sleep promotion. American Journal of Nursing, 104(2), 40-48.

Cohen, L. L., Blount, R. L., Janseevics Cohens, R., Ball, C. M., McClellan, C. B., \& Bernard, R. S. (2001). Children's expectations and memories of acute distress: Short- and long-term efficacy of pain management interventions. Journal of pediatric psychology, 26(6), 367-374.

Colville, G.A. (2004). Parents' views on the psychological impact of admission to PICU. European Psychotherapy,4,81.

Colville, G. (2008). The psychological impact on children of admission to intensive care. Pediatric Clinics of North America, 55(3), 605-616. 
Colville, G. A., \& Gracey, D. (2005). Mothers' recollections of the Paediatric Intensive Care Unit: Associations with psychopathology and views on follow up. Intensive and Critical Care Nursing, 22, 49 - 55. doi: 10.1016/j.iccn.2005.04.002

Colville, G., Kerry, S., \& Pierce, C. (2008). Children's factual and delusional memories of Intensive Care. American Journal of Respiratory Critical Care Medicine, 177(9), 976 - 982. doi: $10.1164 / 4 \mathrm{ccm} 200706$

Colville, G., \& Pierce, C. (2005). Children's memories of pediatric intensive care. Archives of Disease in Childhood, 90(S2), A33-A38.

Colville, G., Tighe, H., \& Pierce, C. (2006). Children's factual and delusional memories of paediatric intensive care. Critical Care Medicine, 34(12), A20.

Committee to Establish Recommended Standards for Newborn ICU Design. (2012). RecStandards for Newborn ICU Design (Eight Consensus Conference on Newborn ICU Design). Retrieved from http://www.nd.edu/ nicudes/stan\%2027.html

Connolly, D., McClowry, S., Hayman, L., Mahony, L., \& Artman, M. (2004). Posttraumatic stress disorder in children after cardiac surgery. Journal of Pediatrics, 144(4) 480-484.

Coyne, I. (2006). Children's experiences of hospitalization. Journal of Child Health Care, 10(4), 326 - 336. doi: 10.1177/1367493506067884

Cremeens, J., Eiser, C., \& Blades, M. (2006). Factors influencing agreement between child self-report and parent proxy-reports on the Pediatric Quality of Life Inventory ${ }^{\mathrm{TM}} 4.0$ (PedsQL ${ }^{\mathrm{TM}}$ ) generic core scales. Health and Quality of Life Outcomes, 4, 58. doi:10.1186/1477-7525-4-58

Cullen, D. J., Civetta, J. M., Briggs, B. A. \& Ferrara, L. C. (1974). Therapeutic intervention scoring system: A method for quantitative comparison of patient care. Critical Care Medicine, 2(2), 57-60.

Curley, M. (1988). Effects of the nursing mutual participation model of care on parental stress in pediatric intensive care unit. Heart Lung. 17. 683-688.

Curley, M.A.Q., \& Wallace, J. (1992). Effects of the nursing mutual participation model of care on parental stress in the pediatric intensive care unit - a replication. Journal of Pediatric Nursing, 7(6), 377-385.

Darbyshire, J. L., \& Young, J. D. (2013). An investigation of sound levels on intensive care units with reference to WHO guidelines. Critical Care, 17(5), R187. Advance online publication. doi: 10.1186/cc12870. 
Davydow, D. S., Richardson, L. P., Zatxick, D. F., \& Katon, W. J. (2010). Psyvhiatric morbidity in pediatric critical illness survivors. Archives of Pediatric and Adolescent Medicine, 164(4), 377-385.

De Los Reyes, A., \& Kazdin, A. E. (2004) Measuring informant discrepancies in clinical child research. Psychological Assessment, 6(3), 330-4.

Dow, B., Kenardy, J., Long, D., \& LeBrocque, R. (2012). Children's post- traumatic stress and the role of memory following admission to intensive care: A review. Clinical Psychologist, 16, 1-14. doi:10.1111/j.1742-9552.2012.00040.x

Elander G., \& Hellström, G. (1999). Reduction of noise levels in intensive care units for infants: Evaluation of an intervention program. Heart Lung: The Journal of Acute and Critical Care, 24(5):376-9.

Ely, E.W., Inouye, S.K., Bernard, G.R., Gordon, S., Francis, J., May, L.,...Dittus, R. (2001). Delirium in mechanically ventilated patients: validity and reliability of the confusion assessment method for the intensive care unit (CAM-ICU). JAMA, 286(21), 2703-2710.

Environmental Protection Agency. Office of Noise Abatement and Control. (1974). Information on levels of environmental noise requisite to protect public health and welfare with an adequate margin of safety (Report no. 5509-74-004).

Washington DC: Government Printing Office.

Fontaine, D. K., Briggs, L. P., \& Pope-Smith, B. (2001) Designing humanistic critical care elements. Critical Care Nursing Quarterly, 24(3), 21-31.

Forsner, M., Jansson, L., \& Sorlie, V. (2005). The experience of being ill as narrated by hospitalized children aged 7-10 years with short term illness. Journal of Child Health Care, 9(2), 153- 165. doi:10.1177/1367493505051406

Gershoff, E. T., Lansford, J. E., Sexton, H. R., Davis-Kean, P., \& Sameroff, A. J. (2012). Longitudinal links between spanking and children's externalizing behaviors in a national sample of White, Black, Hispanic, and Asian American families. Child Development, 83(3), 838-843. doi: 10.1111/j.1467-8624.2011.01732.x

Giusti, G. D., \& Piergentili, F. (2009). Noise in the intensive care unit: A summary review. Connect the World of Critical Care Nursing, 7(1).

Retrieved from http://www.thefreelibrary.com/Noise+in+the+intensive+care+unit $\% 3 \mathrm{~A}+\mathrm{a}+$ summa ry+review\%2FRuido+en+la+unidad...-a0206790217 
Gottschalk, L. A., \& Gleser, G. C. (1969). The measurement of psychological states through the content analysis of verbal behaviour. Berkeley, CA:

University of California Press.

Griffiths, M. (2005). Video games and heath. British Medical Journal, 331(7509), 122-123. doi.org/10.1136\%2Fbmj.331.7509.122

Grover, S., Kate, N., Malhotra, S., Chakrabarti, S., Mattoo, S. K., Avasthi, A. (2012). Symptom profile of delirium in children and adolescents: Does it differ from adults and the elderly? General Hospital Psychiatry, 34(6), 626-632.

Haines, C., Perger, C., \& Nagy, S. (1995). A comparison of the stressors experienced by parents of intubated and non-intubated children. Journal of Advanced Nursing, $21(2) 350-355$.

Hockenberry MJ, Wilson D. Wong's Nursing Care of Infants and Children. 9th ed. St Louis, MO: Mosby; 2011.

Holloway, I., \& Wheeler, S. (2010). Data analysis: Procedures, practices and computers. Qualitative research in nursing and healthcare ( $3^{\text {rd }}$ ed., pp. 281-296). West Sussex, UK: Wiley-Blackwell.

Horowitz, M. J., Wilner, N., \& Alvarez, W. (1979). Impact of event scale: A measure of subjective stress. Psychosomatic Medicine, 41(3), 209-218.

Huckabay, L., \& Tilem-Kessler, D. (1999). Patterns of parental stress in PICU emergency admission. Dimensions in Critical Care Nursing, 18(2), 36-42.

Institute for Healthcare Improvement. (2014). Healthcare associated infections. Retrieved from http://www.ihi.org/topics/HAI/Pages/default.aspx

Irwin, L. G., \& Johnson, J. (2005). Interviewing young children: Explicating our practices and dilemmas. Quality Health Research, 15(6), 823 - 830. doi: $10.1177 / 1049732304273862$

Jayashree, M., Singhi, S. C., \& Malhi, P. (2003). Follow-up of survival and quality of life in children after intensive care. Indian Pediatrics, 40(4), 303-309.

Johnson, P. A., Nelson, G. I., \& Brunnquell, D. J. (1988). Parent and nurse perceptions of parent stressors in the pediatric intensive care unit. Child Health Care, 17(1), $98-105$. 
Johnston, C. C., Bournaki, M. C., Gagnon, A. J., Pepler, C. J., \& Bourgault, P. (2005). Self-reported pain intensity and associated distress in children aged 4-18 years on admission, discharge, and one-week follow up to emergency department. Pediatric Emergency Care, 21(5), 342-346.

Johnston, C. C., Rennick, J. E., Filion, F., Campbell, M., Goulet, C., Bell, L., Tucci, M., \& Ranger, M. (2012). Maternal touch and talk for invasive procedures in infants and toddlers in the pediatric intensive care unit. Journal of Pediatric Nursing, 27(2), 144-153. doi: 10.1016/j.pedn.2010.12.016

Jones, C., Griffiths, R. D., Humphris, G., \& Skirrow, P. (2001). Memory, delusions, and the development of acute posttraumatic stress disorder-related symptoms after intensive care. Critical Care Medicine, 29(3), 687-688.

Jones, S., Rantell, K., Stevens, K., Colwell. B., Ratcliffe, J. R., Holland, P.,... \& Parry, G. J. (2006). Outcome at 6 months after admission for pediatric intensive care: A report of a national study of pediatric intensive care units in the United Kingdom. Pediatrics, 118(5), 2101-2108. doi: 10.1542/peds.2006-1455

Judge, D., Nadel, S., Vergnaud, S., \& Garralda, M. E. (2002). Psychiatric adjustment following meningococcal disease treatment on a PICU. Intensive Care Medicine, 28(5), 648-650. doi: 10.1007/s00134-002-1237-2

Kahn, D. M., Cook, T. E., Carlisle, C. C., Nelson, D. L., Kramer, N. R., \& Millman, R. P. (1998). Identification and modification of environmental noise in an ICU setting. Chest, 114(2), 535-561.

Kain, Z., Hofstadter, M., Mayes, L., Krivutza, D., Alexander, G., Wang, S., \& Reznick, J. (2000). Midazolam: Effects on amnesia and anxiety in children. Anesthesiology, 93, 676- 684 .

Kalish, C. (1996). Preschoolers' understanding of germs as invisible mechanisms. Cognitive Development, 11(1), 83-106.

Kalish, C. (1997). Preschoolers' understanding of mental and bodily reactions to contamination: What you don't know can hurt you, but cannot sadden you. Developmental Psychology, 33(1), 79-91.

Karande, S., Kelkar, A., \& Kulkarni, M. (2005). Recollections of Indian children after discharge from an intensive care unit. Pediatric Critical Care Medicine, 6(3), 303-7.

Kato, P. M., Cole, S. W., Bradlyn, A. S., \& Pollock, B. (2008). A video game improves behavioral outcomes in adolescents and young adults with cancer. A randomized trial. Pediatrics, 122, e305-e317. doi: 10.1542/peds.2007-3134 
Keene, A. R., Cullen, D. J. (1983). Therapeutic intervention scoring system: Update. Critical Care Medicine, 11(1), 1-3.

Kellam, B. C., \& Bhatia, J. (2008). Sound spectral analysis in the NICU: High frequency noise. Journal of Pediatric Nursing, 23(4), 317-323.

doi: 10.1016/j.pedn.2007.09.009

Klein, V. C., Gaspardo, C. M., Martinez, F. E., Grunau, R. E., \& Linhares, M. B. (2009). Pain and distress reactivitya nd recoverya s early predictors of temperamnet in toddlers born preterm. Early Human Development, 85(9), 569-576. doi:10.1016/j.earlhumdev.2009.06.001

Koening, K., Chesla, C., \& Kennedy, C. (2003) Parent's perspectives of asthma crisis hospital management in infants and toddlers: An interpretive view through the lens of attachment theory. Journal of Pediatric Nursing, 18(4) 233-242.

Koopman, H. M., Baars, R. M., Chaplin, J., \& Zwinderman, K. H. (2004).Illness through the eyes of the child: The development of children's understanding of the cause of illness. Patient Education and Counseling, 53(3), 363-370.

doi:10.1016/j.pec.2004.02.020

Landolt, M. A., Buchlmann, C., Maag, T., \& Schiestl, C. (2009). Brief report: Quality of life is impaired in pediatric burn survivors with posttraumatic stress disorder. Journal of Pediatric Psychology, 34(1), 14-21. doi: 10.1093/jpepsy/jsm088

Lasky, R. E., \& Williams, A. L. (2009). Noise and light exposures for extremely low birth weight newborns during their stay in the Neonatal Intensive Care Unit. Pediatrics, 123(2), 540-546. doi:10.1542/peds.2007-3418

Lawson, N., Thompson, K., Saunders, G., Saiz, J., Richardson, J., Brown, D., Ince, N., Caldwell, M., \& Pope, D. (2010). Sound intensity and noise evaluation in a critical care unit. American Journal of Critical Care, 19(6), E88-E99. doi: $10.4037 /$ ajcc2010180

Lazarus, R. S. (2012). Evolution of a model of stress, coping, and discrete emotions. In V. H. Rice (Ed), Handbook of stress, coping, and health: Implications for nursing research, theory, and practice (pp. 199 - 225).Thousand Oaks, CA: Sage.

Lee, M. D., Friedenberg, A. S., Mukpo, D. H., Conray, K., Palmisciano, A., \& Levy, M. M. (2007). Visiting hours policies in New England intensive care units: Strategies for improvement. Critical Care Medicine, 35(2), 497-501. doi: 10.1097/01.CCM.0000254338.87182.AC

Lee, Y.-H., Malakooti, N., \& Lotas, M. (2005). A comparison of the light-reduction capacity of commonly used incubators covers. Neonatal Network, 24(2), 37-44. 
Levy, M. C., Kronenberger, W. G., \& Carter, B. D. (2008). Brief report: Illness factors and child behavior before and during pediatric hospitalization. Journal of Pediatric Psychology, 33(8), 905-909.

Lindke, L., Nakai, M., \& Johnson, L. (2006) Capturing children's voices for quality improvement. The American Journal of Maternal Child Nursing, 31, 290-295.

Logsdon, M., Birkimer, J., Ratterman, A., Cahill, K., \& Cahill, N. (2002). Social support in pregnancy and parenting adolescents: Research, critique, and recommendations. Journal of Child and Adolescent Psychiatric Nursing, 15, $75-84$.

Long, L. E. (2003). Stress in families of children with sepsis. Critical Care Nursing Clinics of North America, 15(1), 47-53.

Long, J. G., Lucey, J. F., \& Philip, A. G. (1980). Noise and hypoxemia in the intensive care nursery. Pediatrics, 65(1), 143-145.

Lusk, B. \& Lash, A. A. (2005). The stress response, psychoneuroimmunology, and stress response among ICU patients. Dimensions of Critical Care Nursing, 24(1), $25-31$.

Magee, T., \& Roy, S. C. (2008). Predicting school-age behavior problems: The role of early childhood risk factors. Pediatric Nursing, 34(1), 37 -44.

Marino, B. S., Tomlinson, R. S., Drotar, D., Clayborn, E. S., Aguirre, A., Ittenbach, R., \& Shea, J. (2009). Quality-of-life concerns differ among patients, parents, and medical providers in children and adolescents with congenital and acquired heart disease. Pediatrics, 123(4), e708 - 715. doi: 10.1542/peds.2008-2572

Matook, S. A., Sullivan, M. C., Saunders, G., Miller, R. J., \& Lester, B. M. (2010). Variations of NICU sound by location and time of day. Neonatal Network: Journal of Neonatal Nursing, 29(2), 87-95.

McQuaid, E. L., Howard, K., Kopel, S. J., Rosenblum, K., \& Bibace, R. (2002). Developmental concepts of asthma: Reasoning about illness and strategies for prevention. Applied Developmental Psychology, 23, 179-194.

Meagher, D. (2009). Motor subtypes of delirium: Past, present, and future. International Review of Psychiatry, 21(1), 59-73. doi: 10.1080/09540260802675460

Meagher, D. J., \& Trzepacz, P. T. (2000). Motor subtypes of delirium. Seminars in Clnical Psychiatry, 5(2), 75-85. 
Melnyk, B. M. (1994). Coping with unplanned childhood hospitalization: Effects of informational interventions on mothers and children. Nursing Research, 43, $50-55$.

Melnyk, B. M. (2000). Intervention studies involving parents of hospitalized young children: An analysis of the past and future. Journal of Pediatric Nursing, 15(1), 4-13.

Melnyk, B. M., Alpert-Gillis, L., Feinstein, N. F., Crean, H. F., Johnston, J., Fairbanks, E.,...Corbo-Richert, R. (2004). Creating opportunities for parent empowerment: Program effects on the mental health/coping outcomes of critically ill young children and their mothers'. Pediatrics, 113(6), e597 - e607.

Melnyk, B. M., \& Feinstein, N. F. (2001). Mediating functions of maternal anxiety and participation in care on young children's post-hospital adjustment. Research in Nursing and Health, 24(1), 18-26. doi: 10.1002/1098-240x(200102)

Miles, M. S., \& Carter, M. C. (1982). Sources of parental stress in pediatric intensive care units. Children's Health Care, 11(2), 65-69.

Miles, M. S. \& Carter, M. C. (1989). The Parental Stressor Scale: Pediatric Intensive Care Unit. Maternal-Child Nursing Journal, 18(3), 187- 198.

Miles, M. B., Huberman, A. M., \& Saldaña, J. (2014). Qualitative data analysis: A methods sourcebook ( ${ }^{\text {rd }}$ ed.). Thousand Oaks, CA: Sage.

Milette, I. H., \& Carnevale, F. A. (2003). I'm trying to heal... Noise levels in a pediatric intensive care unit. Dynamics, 14(4), $14-21$.

Miner, J. R., Bachman, A., Kosman, L., Teng, B., Heegard, W., \& Biros, M. H. (2005). Assessment of onset and persistence of amnesia during procedural sedation with procedural sedation with propofol. Academic Emergency Medicine, 31(1), 113-119.

Morandi, A., Jackson, J. C., Ely, E. W. (2009). International Review of Psychiatry, 21(1), 43-58. doi: 10.1080/09540260802675296

Morrison, W. E., Haas, E. C., Shaffner, D. H., Garrett, E. S., \& Fackler, J. C. (2003). Noise, stress, and annoyance in a pediatric intensive care unit. Critical Care Medicine, 11(1),113-119. doi: 10.1097/01.CCM.0000037164.66392.AF

Morrow, B. M. (2008). A comprehensive review of pediatric endotracheal suctioning: Effects, indicators, and clinical practice. Pediatric Critical Care Medicine, 9(5), 465-477. doi: 10.1097/PCC.0b013e31818499cc 
Muranjan, M. N., Birajdar, S. B., Shah, H. R., Sundaraman, P., \& Tullu, M. S. (2008). Psychological consequences in pediatric intensive care unit survivors: The neglected outcome. Indian Pediatrics, 45(3), 99 - 103.

Myant, K. A., \& Williams, J. M. (2005). Children's concepts of health and illness: Understanding of contagious illness, non-contagious illnesses and injuries. Journal of Health Psychology, 10(6), 805-819. doi: 10.1177/1359105305057315

Namachivayam, P., Shann, F., Shekerdemian, L., Taylor, A., van Sloten, I., Delzoppo, C. \& Warwick, B. (2010). Three decades of pediatric intensive care: Who was admitted, what happened in intensive care, and what happened afterward. Pediatric Critical Care Medicine, 11(5), 549-555. doi: 10.1097/PCC.0b013e3181ce7427

Odetola, F. O., Clark, S. J., Freed, G. L., Bratton, S. L., \& Davis, M. M. (2005). A national survey of pediatric critical care resources in the United States. Peditarics, 115(4), e382-386. doi: 10.1542/peds.2004-1920

Ouimet, S. Kavannagh, B. P., Gottfied, S. B., \& Skrobik, Y. (2007). Incidence, risk factors, and consequences of ICU delirium. Intensive Care Medicine, 33(1), 66-73.

Pandharipande, P., Shintani, A., Peterson, J., Pun, B. T., Wilkinson, G. R., Dittus, R. S., Bernard, G. R., \& Ely, E. W. (2006). Lorazepam is an independent risk factor for transitioning to delirium in intensive care unit patients. Anesthesiology, 104(1), 21-26.

Pelander, T., Leino-Kilpi, H., \& Katajisto, J. (2006). Quality of pediatric nursing care in Finland: Children's perspective. Journal of Nursing Care Quality, 22(2), 185-194.

Peltzer, K., \& Promtussananon, S. (2003). Black South African children's understanding of health and illness: Colds, chicken pox, broken arms, and AIDS. Child: Care, Health and Development, 29(5), 385-393.

Pickhardt, C. (2013). A road map to early and mid-adolescence. Surviving your child's adolescence: How to understand, and even enjoy, the rocky road to independence (pp. 21-49). Hoboken, NJ: Wiley

Piko, B. F., \& Bak, J. (2006). Children's perceptions of health and illness: Images and lay concepts in preadolescence. Health Education Research, 21(5), 643 - 653. doi:10.1093/her/cy1034

Playfor, S., Thomas, D., \& Choonara, I. (2000). Recollections of children following intensive care. Archives of Disease in Childhood, 83(5), 445 - 448.

doi: $10.1136 /$ adc.83.5.445 
Pisani, M. A., Murphy, T. E., Araujo, K. L., Slattum, P., Van Ness, P. H., Inouye, S. K. (2009). Benzodiazepine and opiod use and the duration of intensive care unit delirium in an older population. Critical Care Medicine, 37(1), 177-183. doi: 10.1097/CCM.0b013e318192fcf9

Playfor, S., Thomas, D., \& Choonara, I. (2000). Recollections of children following intensive care. Archives of Disease in Childhood, 83(5), 445 - 448. doi: 10.1136/adc.83.5.445

Poling, D. A., \& Evans, E. M. (2004). Are dinosaurs the rule or the exception? Developing concepts of death and extinction. Cognitive Development, 19(3), $363-383$.

Poltorak, D. Y., \& Glazer, J. P. (2006). The development of children's understanding of death: cognitive and psychodynamic considerations. Child and Adolescent Psychiatric Clinics of North America, 15(3), 567-573.

Prehn-Kristensen, A., Göder, R., Chirobeja, S., Bressmann, I., Ferstl, R., \& Baving, L. (2009). Sleep in children enhances preferentially emotional declarative but not procedural memories. Journal of Experimental Child Psychology, 104(1), 132-139. doi: 10.1016/j.jccp.2009.01.005

Pringle, B., Dahlquist, L. M., \& Eskenazi, A. (2003). Memory in pediatric patients undergoing conscious sedation for aversive medical procedures. Health Psychology, 22(3), 263-269.

Quaraishi, S. A., Girdharry, T. D., Xu, S.-G., \& Orkin, F. K. (2007). Prolonged Retrograde amnesia following sedation with propofol in a 12-year-old boy. Pediatric Anesthesia, 17(4), 375-379.

Rajmil, 1., López, A. R., López-Aguilà, S., \&Alonso, J. (2013). Parent-child agreement on health-related quality of life (HRQOL): A longitudinal study. Health and Quality of Life, 11(1), 101. doi:10.1186/1477-7525-11-101

Ramen, L., \& Winer, G. A. (2002). Children's and adults' understanding of illness: Evidence in support of a coexistence model. Genetic, Social, and General Psychology Monographs, 128(4), 325-355.

Ratcliffe, S., Meyer, W., Cuervo, L., Villarreal, C., Thomas, C., \& Herndon, D. (2004). The use of haloperidol and associated complications in the agitated acutely ill paediatric burn patient. Journal of Burn Care Rehabilitation, 25(6), 472-478.

Rees, G., Gledhill, J., Garralda, M. E., \& Nadel, S. (2004). Psychiatric outcome following paediatric intensive care unit (PICU) admission: A cohort study. Intensive Care Medicine, 30(8), 1607 - 1614. doi: 10.1007/s00134-004-2310-9 
Rennick, J. E. (1986). Reestablishing the parental role in the pediatric intensive care unit. Journal of Pediatric Nursing, 1(1), 40-44.

Rennick, J. E., \& Johnston, C. C. (2000). Development of measure to examine posttraumatic stress responses in children following critical illness. Pediatric Critical Care Medicine, 1(supp 1), 60.

Rennick, J. E., Johnston, C., Dougherty, G., Platt, R., \& Ritchie, J. A. (2002). Children's psychological responses after critical illness and exposure to invasive technology. Developmental and Behavioral Pediatrics, 23(3), 133 - 144.

Rennick, J. E., Johnston, C., Lambert, S. D., Rashotte, J. M., Schmitz, N., Earle, R. J.,... Wood-Dauphinee, S. (2011). Measuring psychological outcomes following pediatric intensive unit hospitalization: Psychometric analysis of the Children's Critical Illness Impact Scale. Pediatric Critical Care Medicine, 12(6), 635 - 642. doi:0.1097/PCC.0b013e3182191bfa

Rennick, J. E., McHarg, L.F., Dell'Api, M., Johnston, C. C., \& Stevens, B. (2008). Developing the Children's Critical Illness Impact Scale: Capturing stories from children, parents, and staff. Pediatric Critical Care Medicine, 9(3), 252 - 260. doi: 10.1097/PCC.0b013e31816c70d4

Rennick, J. E., Morin, I., Kim, D., Johnston, C., Dougherty, G., \& Platt, R. (2004). Identifying children at high risk for psychological sequelae after pediatric intensive care unit hospitalization. Pediatric Critical Care Medicine, 5(4), 358 - 363. doi: 10.1097/01.PCC.0000128603.20501.00

Rennick, J. E., \& Rashotte, J. (2009). Psychological outcomes in children following pediatric intensive care unit hospitalization: A systematic review of the research. Journal of Child Health Care, 13, 128 -149. doi:10.1177/1367493509102472

Rhoney, D. H., \& Parker, D. (2001). Use of sedative and analgesic agents in neurotrauma patients: Effects on cerebral physiology. Neurological Research, 23(2-3), 237-259.

Russell, K. M. W., Hudson, M., Long, A., \& Phipps, S. (2006). Assessment of healthrelated quality of life in children with cancer. Cancer, 106 (10):2267-74.

Ryan-Wenger, N. A. (1992). A taxonomy of children's coping strategies: A step toward theory development. American Orthopsychiatric Association, 62(2), 256-263.

Ryan-Wenger, N. A. (1996). Children, coping, and the stress of illness: A synthesis of the research. Journal of the Society of Pediatric Nurses, 1(3), 126 - 138. doi: 10.1111/j.1744-6155.1996.tb00015.x 
Ryan-Wenger, N. A., \& Gardner, W. (2012). Hospitalized children's perspectives on the quality and equity of their nursing care. Journal of Nursing Care Quality, 27(1), 35-42. doi: 10.1097/NCQ.0b013e31822f71a3

Salmela, M., Salanterä, S. \& Aronen, E. T. (2009) Child-reported hospital fears of 4 to 6 year-old children. Pediatric Nursing, 5, 269-276.

Sameroff, A. (2009). The transactional model. In Arnold Sameroff (Ed.), The transactional model of development: How children and contexts shape each other (pp. 3-20). Washington DC: American Psychological Association.

Sameroff, A.J., \& Chandler, M.J. (1975). Reproductive risk and the continuum of caretaking causality. In F. D. Horowitz, E. M. Hetherington, S. Scarr-Salapatek, \& G. Siegel (Eds.), Review of Child Development Research (Vol. 4, pp 187-244). Chicago: University of Chicago Press.

Scheeringa, M. S., Zeanah, C. H., \& Cohen, J. A. (2011). PTSD in children and adolescents: Toward an empirically based algorithm. Depression and Anxiety, 28(9), 770-782.

Schmidt, C., Bernaix, L., Koski, A., Weese, J., Chiappetta, M., \& Sandrik, K. (2007). Hospitalized children's perceptions of nurses and nurse behaviors. $M C N$, American Journal of Maternal and Child Nursing, Nov-Dec. 32(6), 336-342. doi: 10.1097/01.NMC.0000298126.32161.4c

Schieveld, J. N. M., Leroy, P. L. J. M., van Os, J., Nicolai, J., Vos, G. D., \& Leentjens, A. F. G. (2007). Pediatric delirium in critical illness: Phenomenology, clinical correlates and treatment response in 40 cases in the pediatric intensive care unit. Intensive Care Medicine, 33(6), 1033-1040. doi: 10.1007/s00134-007-0637-8

Scragg, P., Jones, A., \& Fauvel, N. (2001). Psychological problems following ICU treatment. Anaesthesia, 56(1):9-14.

Seng, J. S., Graham-Bermann, S. A., Clark, C. R., McCarthy, A. M., \& Ronis, D. L. (2005). Posttraumatic stress disorder and physical comorbidity among female children and adolescents: Results from service-use data. Pediatrics, 116(6), 767-776.

Shann, F., Pearson, G., Slater, A., \& Wilkinson, K. (1997). Paediatric index of mortality (PIM): A mortality prediction model for children in intensive care.

Intensive Care Medicine, 23(2), 201-207. 
Sheffler, L. C., Hanley, C., Bagley, A., Molitor, F., \& James, M. A. (2009). Comparison of self-reports and parent proxy-reports of function and quality of life of children with below-the-elbow deficiency. The Journal of Bone and Joint Surgery, 91(12), 2852-2859. doi: 10.2106/JBJS.H.01108

Shudy, M., Lihinie de Almeida, M., Ly, S., Landon, C., Groft, S., Jenkins, T. L., \& Nicholson, C. E. (2006). Impact of pediatric critical illness and injury on families: A systematic literature review [Supplement 3], Pediatrics, 118, S 203-218. doi: 10.1542/peds.2006-0951B

Slaughter, V., \& Griffiths, M. (2007). Death understanding and fear of death in young children. Clinical Child Psychology and Psychiatry, 12(4), 525-535.

Slaughter, V, M., \& Lyons., M. (2002). Learning about life and death in early childhood. Cognitive Psychology, 46(1), 1-30. doi: 10.1016/S0010-0285(02)00504-2

Small, L., \& Melnyk, B. M. (2006). Early predictors of post-hospital adjustment problems in critically ill children. Research in Nursing \& Health, 29, $622-635$. doi:10.1002/nur.20169

Smeets, I. A., Tan, E. Y., Vossen, H. G., Leroy, P. L., Lousberg, R. H., van Os J., \& Schieveld, J.N. (2010). Prolonged stay at the paediatric intensive care unit associated with paediatric delirium. European Child and Adolescent Psychiatry, 19(4), 389-93. doi: 10.1007/s00787-009-0063-2

Smith, A. B., Hefley, G. C., \& Anand, K. J. S. (2011). Parent bed spaces in the PICU: Effect on parental stress. Pediatric Nursing, 33(3), 215-221.

Starkweather, A., Witek-Janusek, L., \& Mathews, H. L. (2005).Applying the psychoneuroimmunology framework to nursing research. Journal of Neuroscience Nursing, 37(1), 56-62.

Stewart, S., Buffett-Jerrott, S., Finley, G., Wright, K., \& Valois Gomez, T. (2006). Effects of midazolam on explicit vs implicit memory in a pediatric surgery setting. Psychopharmacology, 188(4), 489-497. doi: 10.1007/s00213-006-0402-7

Stormshak, E. A., Bierman, K. L., McMahon, R. J., \& Lengua, L. J. (2000). Parenting practices and child disruptive behavior problems in early elementary school. Journal of Clinical Child and Adolescent Psychology, 29(1), 17-29.

Sundblad, G. M. B., Saartok, T., \& Engström, L.-M. T. (2006). BMC Public Health, 6, 276. doi: 10.1186/1471-2458-6-276.

Szczepanski, M., \& Kamianowska, M. (2008). The illumination intensity in the neonatal intensive care unit. Archives of Perinatal Medicine, 14, 47-50. 
Taddio, A., Shah, V., Gilbert-MacLeod, C. \& Katz, J. (2002). Conditioning and hyperalgesia in newborns exposed to repeated heel lances. JAMA, 288(7), 857861.

Taylor, A., Butt, W., \& Ciardulli, M. (2003). The functional outcome and quality of life of children after admission to an intensive care unit. Intensive Care Medicine, 29(5), 795- 800. doi: 10.1008/s00134-003-01690-6

Taylor-Ford, R., Caitlin, A., LaPlante, M., \& Weinke, C. (2008). Effect of a noise reduction program on a medical-surgical unit. Clinical Nursing Research, 17(2), 74-88. doi: 10.1177/1054773807312769

Thompson, V. L., Hupcey, J. E., \& Clark, M. B. (2003). The development of trust in parents of hospitalized children. Journal for Specialists in Pediatric Nursing, 8(4), 137-147. doi: 10.1111/j.1088-145X.2003.00137.x

Tichy, A. M., Braam, C. M., Meyer, T. A., \& Rattan, N. S. (1988). Stressors in pediatric intensive care units. Pediatric Nursing, 14(1), $40-42$.

Trzepacz, P. T. (1999). Update on the neuropathogenesis of delirium. Dementia \& Geriatric Cognitive Disorders, 10, 333-334.

Tune, L., Carr, S., Hoag, E., \& Cooper, T. (1992). Anticholinergic effects of drugs commonly prescribed for the elderly: Potential means for assessing risk of delirium. American Journal of Psychiatry, 149(10), 1393- 1394.

Turkel, S. B., \& Tavare', C. J. (2003). Delirium in children and adolescents. Journal of Neuropsychiatry and Clinical Neurosciences, 15(4), 431 - 435.

Turner, H, N, (2005). Complex pain consultations in the pediatric intensive care unit. AACN Clinical Issues, 16(3), 388-395.

Twite, M. D., Rashid, A., Zuk, J., Friesen, R. H. (2004). Sedation, analgesia, and neuromuscular blockade in the pediatric intensive care unit: Survey of fellowship training programs. Pediatric Critical Care Medicine, 5(6), 521-532.

Vanek, C. W. (1979). How school-age children perceive the intensive care unit environment. Journal of the New York State Nurses' Association, 10(4), 30-33.

Varni, J. W., \& Bernstein, B. H. (1991). Evaluation and management of pain in children with rheumatic diseases. Rheumatic Disease Clinics of North America, 17(4), $985-1000$. 
Varni, J. W., Limbers, C. A., \& Burlwinkle, T. M. (2007a). How young can children reliably and validly self-report their health related quality of life? An analysis of 8,591 children across age subgroups with the Peds QL ${ }^{\mathrm{TM}} 4.0$ Generic Core Scales. Health and Quality of Life Outcomes, 5, 1-13. doi: 10.1186/1477-7525-5-1

Varni, J. W., Limbers, C. A., \& Burlwinkle, T. M. (2007b). Parent proxy-report of their children's health-related quality of life: An analysis of 13,878 parents' reliability and validity across age subgroups using the Peds QLTM 4.0 Generic Core Scales. Health and Quality of Life Outcomes, 5, 2. doi: 10.1186/1477-7525-5-2

Varni, J. W., Seid, M. Kurtin, P. S. (2001). PedsQL TM 4.0: Reliability and validity of the Pediatric Quality of Life Inventor Version 4.0 Generic Core Scales in healthy and patient populations. Medical Care, 39, 800-812.

Varni, J. W., Thompson, K. L. Hanson, V. (1987). The Varni/Thompson Pediatric Pain Questionnaire: I. Chronic musculoskeletal pain in juvenile rheumatoid arthritis. Pain, 28(1), 27 -38.

Veldtman, G. R., Matley, S. L. Kendall, L., Quirk, J., Gibbs, J. L., Parsons, J. M., \& Hewlson, J.(2001). Illness understanding $\mathrm{n}$ children and adolescents with heart disease. Western Journal of Medicine, 174(3), 171-173.

Vetter, T. R., Bridgewater, C. L., \& McGwin, G. Jr. (2012). An observational study of patient versus parental perceptions of health-related quality of life in children and adolescents with a chronic pain condition: Who should the clinician believe? Health and Quality of Life Outcomes, 10, 85. doi:10.1186/1477-7525-10-85

Vlajkovic, G., \& Sindjelic, R. (2007). Emergence delirium in children: Too many questions, few answers. Anesthesia and Analgesia, 104(1), 84-91. doi: 10.1213/01.ane.0000250914.91881.a8

Walder, B., Francioli, D., Mayer, J., Lancon, M., \& Romand, J. (2000). Effects of guidelines implementation in a surgical intensive care unit to control nighttime light and noise level. Critical Care Medicine, 28(7), 2242-2247.

Wennström, L. \& Bergh, I. (2008) Bodily and verbal expressions of postoperative symptoms in 3 - to 6- year-old boys. Journal of Pediatric Nursing, 23(1), 65-76. doi:10.1016/j.pedn.2006.05.013

Wennström L., Hallgerg, R.-M., \& Bergh I. (2008) Use of perioperative dialogue with children undergoing day surgery. Journal of Advanced Nursing, 62(1), 96-106. doi: $10.1111 / \mathrm{j} .1365-2648.2007 .04581 . \mathrm{x}$

WHO Guidelines for community noise. (1999). World Health Organization, Geneva. 
Williams, J. M., \& Binnie, L. M. (2002). Children's concepts of illness: An intervention to improve knowledge. British Journal of Health Psychology, 7, 129-147.

Williams, A. L., van Drongelen, W., \& Lasky, R. E. (2007). Noise in contemporary neonatal intensive care. Journal of Acoustical Society of America, 121(5), 26812690 .

Wilson, M. E., Megel, M. E., Barton, P. H., Bell, J., Marget, A., Ranck, S.,...Wolf, L. (2007). Revision and psychometric testing of the Barton Hospital Picture Test. Journal of Pediatric Nursing, 22(3), 206-214.

Wilson, M. E., Megel, M. E., Enenbach, L., \& Carlson, K. L. (2010). The voices of children: Stories about hospitalization. Journal of Pediatric Health Care, 24(2), 95-102. doi: 10.1016/j.pedhc.2009.02.008

Xie, H., Kang, J., \& Mills, G. H. (2009). Clinical review: The impact of noise on patients' sleep and the effectiveness of noise reduction strategies in intensive care units. Critical Care, 13(2), 208-216. doi: 10.1186/cc7154

Youngblut, J. (1994). Children's understanding of illness: Developmental concepts. AACN Clinical Issues in Critical Care Nursing, 5(1), 42- 48.

Youngblut, J. M., Brooten, D. (2006). Pediatric head trauma: Parent, parent-child and family functioning 2 weeks after hospital discharge. Journal of Pediatric Psychology, 31(6), 608-618.

Youngblut, J. M., \& Brooten, D. (2008). Mother's mental health, mother-child relationship, and family functioning 3 months after a preschoolers head injury. Journal of Head Trauma and Rehabilitation, 23(2), 92- 102.

Youngblut, J. M., \& Brooten, D. (2014). Parent's report of child's response to sibling death in a Neonatal or Pediatric ICU. American Journal of Critical Care, 22(6), 474-481. doi: 10.4037/ajcc2013790

Youngblut, J.M., Brooten, D., \& Kuluz, J. (2005). Parent reactions at 24-48 hours after a preschool child's head injury. Pediatric Critical Care Medicine, 6(5), 550-556.

Zaal, I. J., \& Slooter A. J. C. (2012). Delirium in critically ill patients: Epidemiology pathophysiology, diagnosis and management. Drugs, 72(11), 1457-1471.

Zengerle-Levy, K. (2005). Nursing the child who is alone in the hospital. Pediatric Nursing, 32(3), 226-231. 
Zhu, L., \& Fang, F. (2000). Development of Chinese preschoolers' understanding of biological phenomena: Growth and aliveness. International Journal of Behavioral Development,24, 105-110. doi: 10.1080/016502500383539 
Appendix A

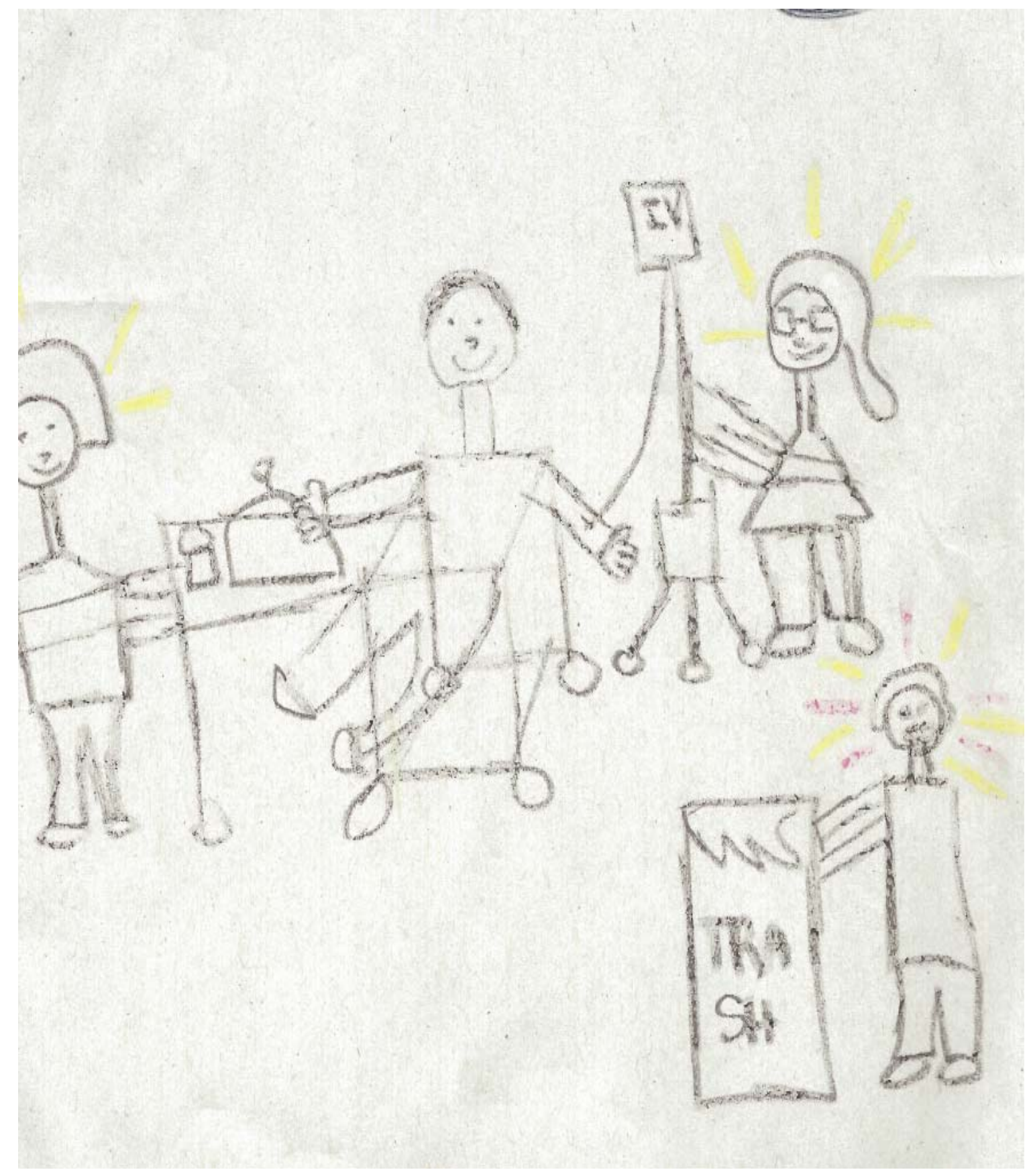


Appendix B
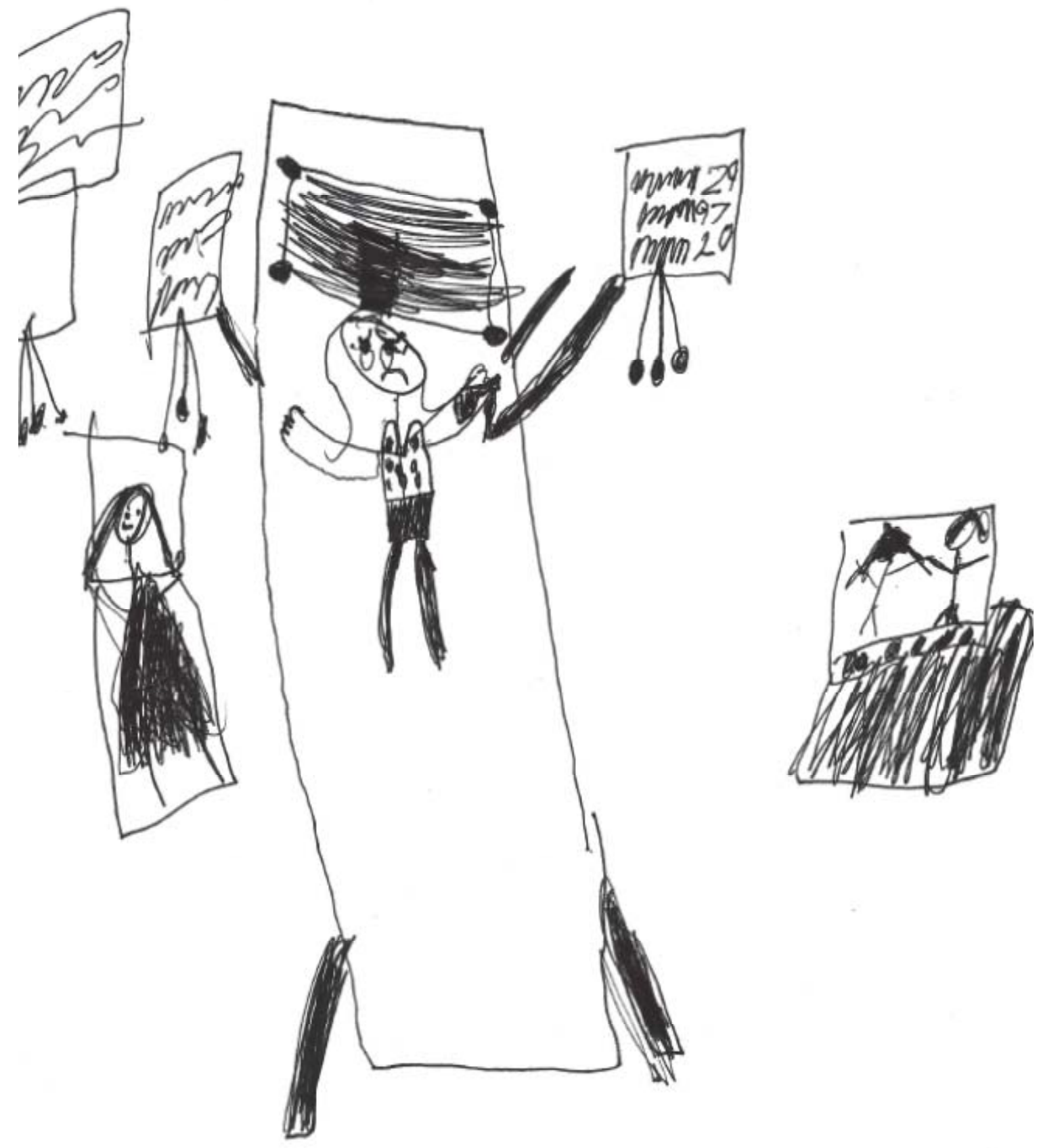
Appendix C

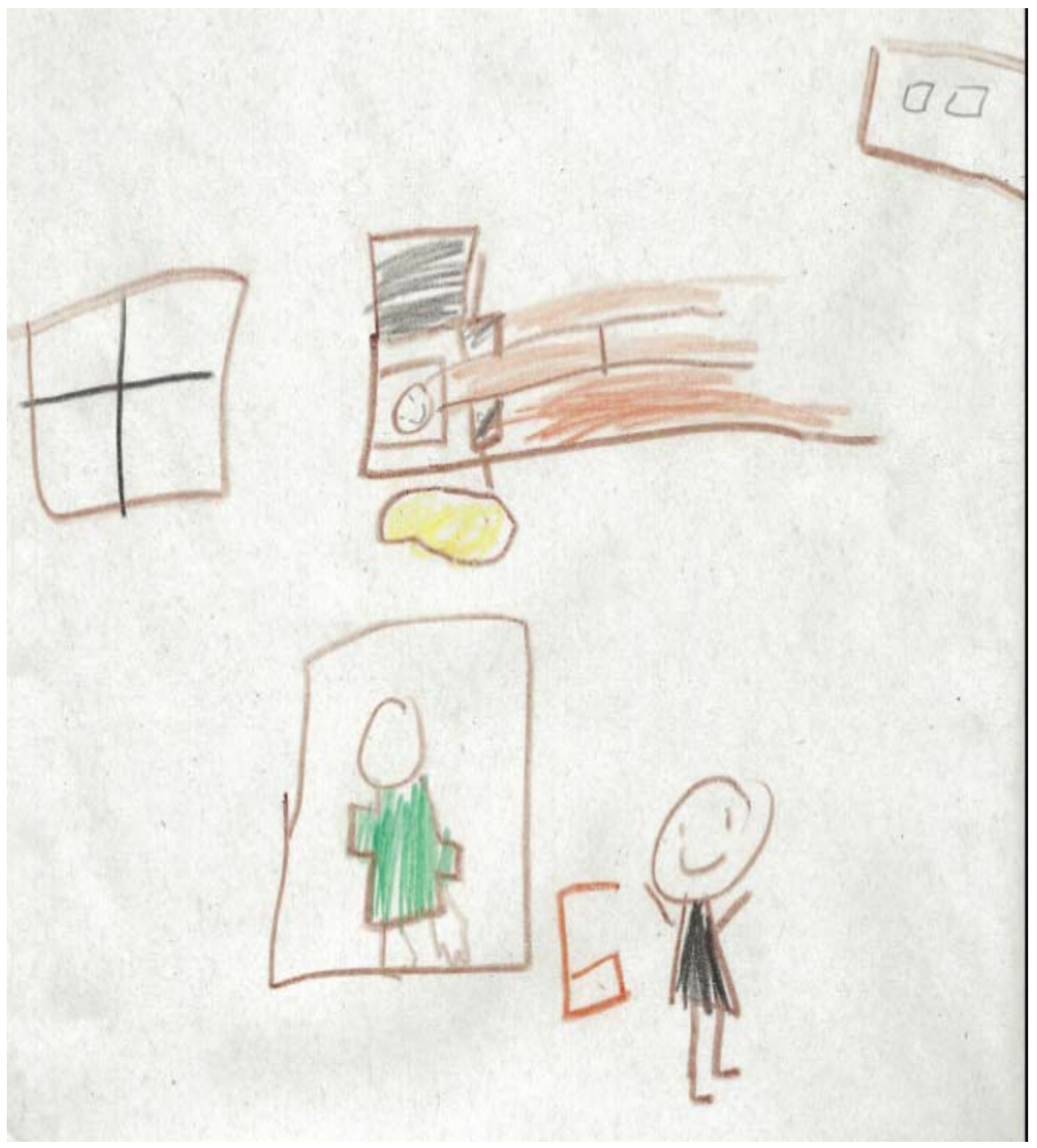


Appendix D

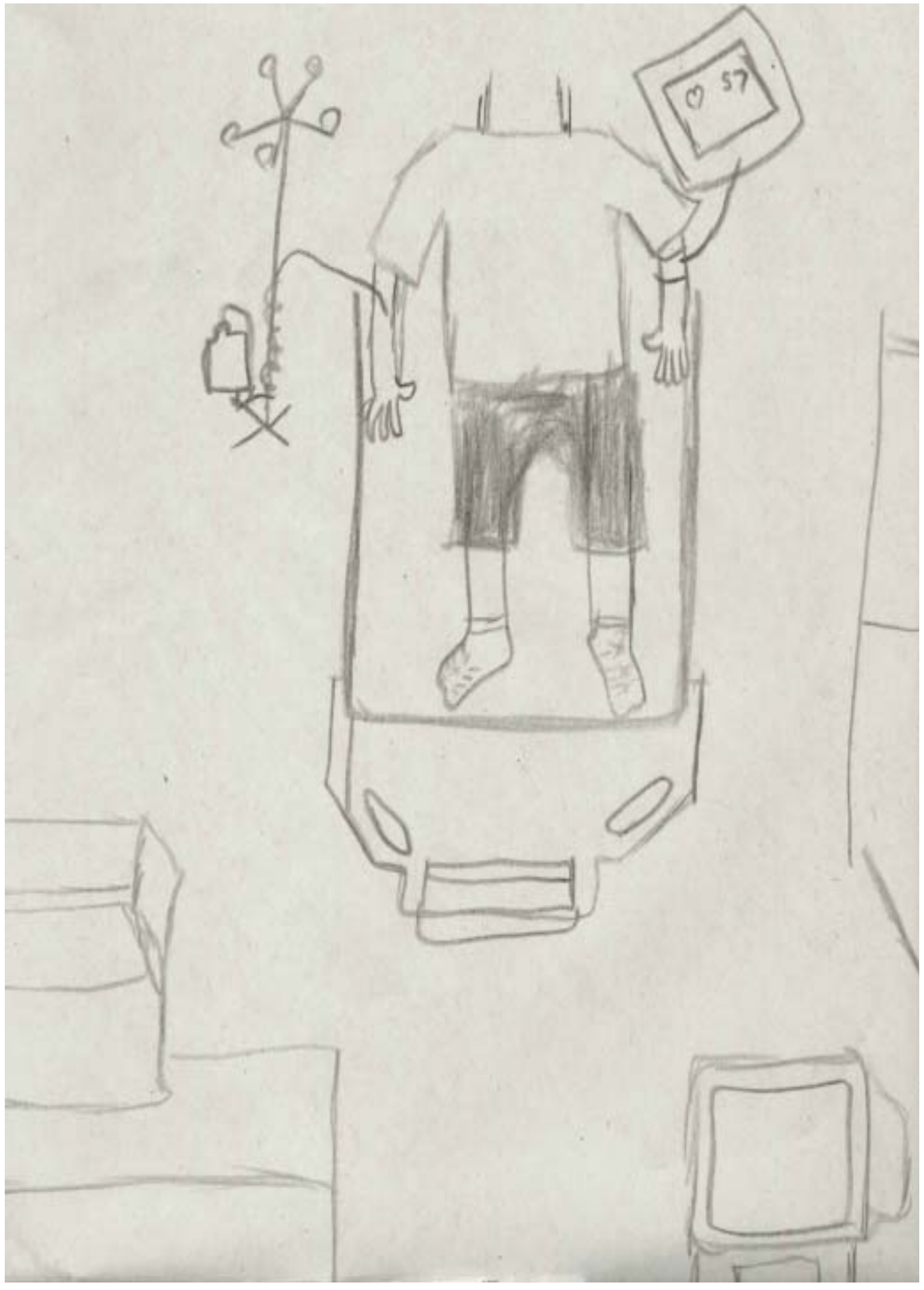


Appendix E

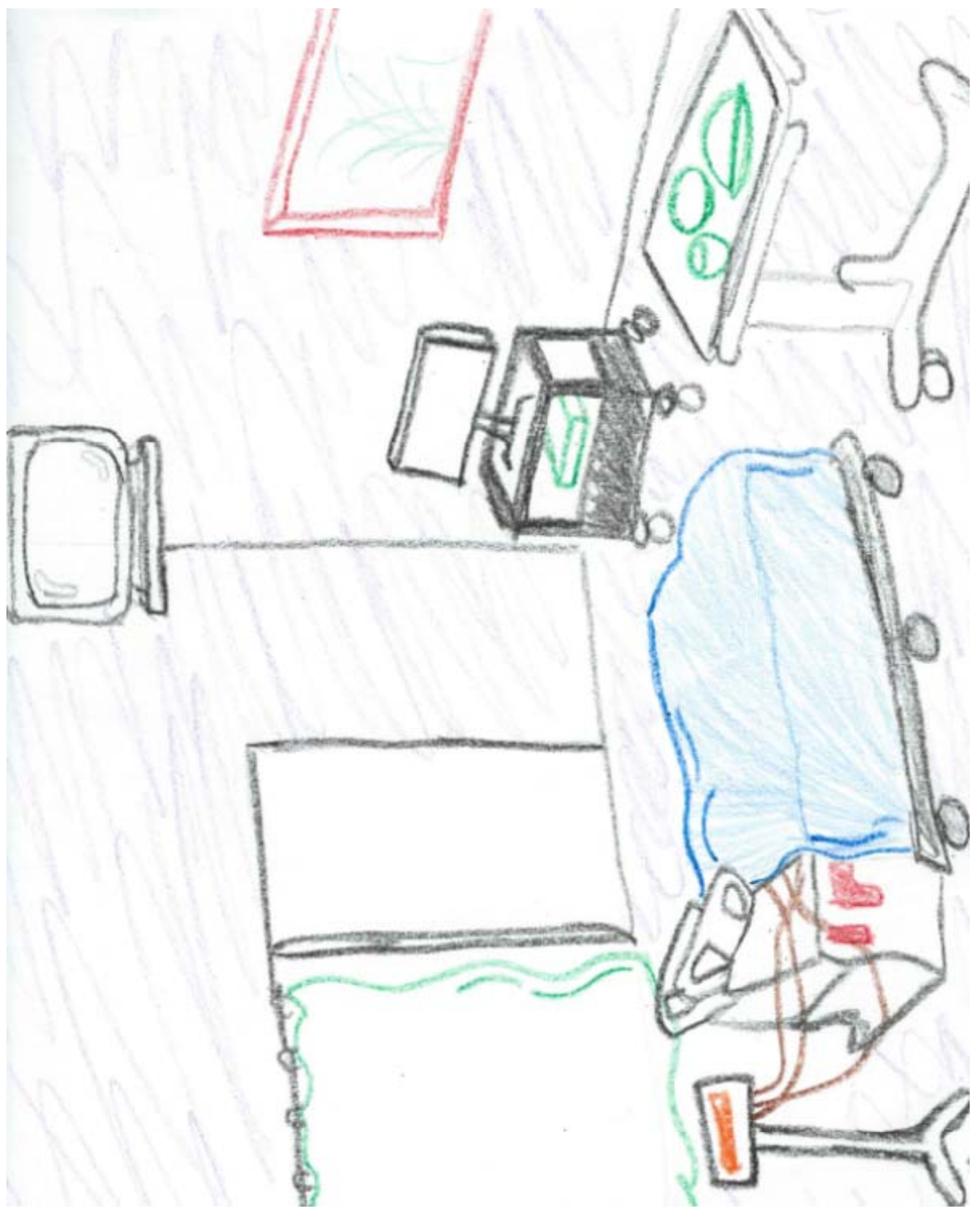


Appendix F

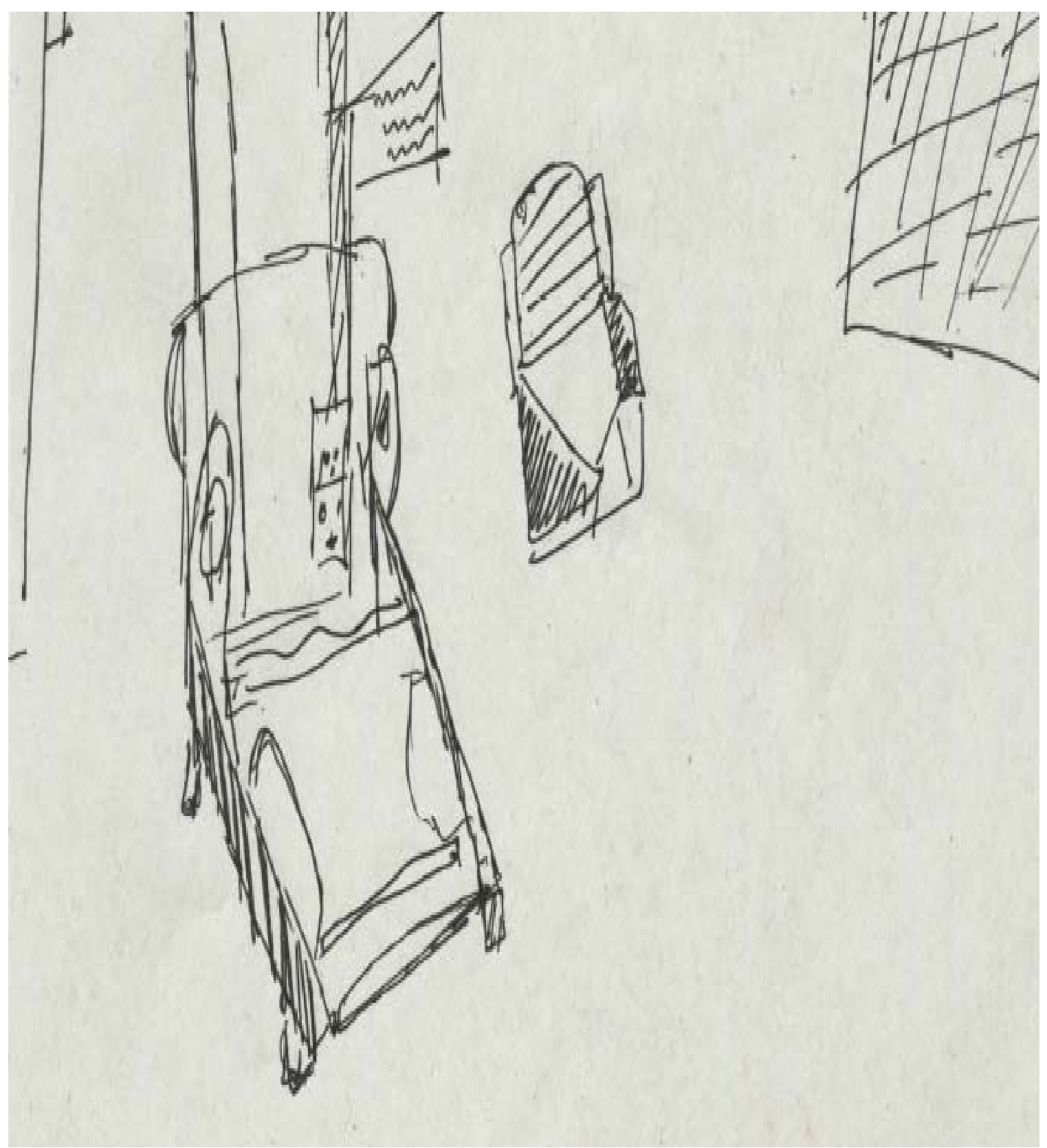


Appendix G

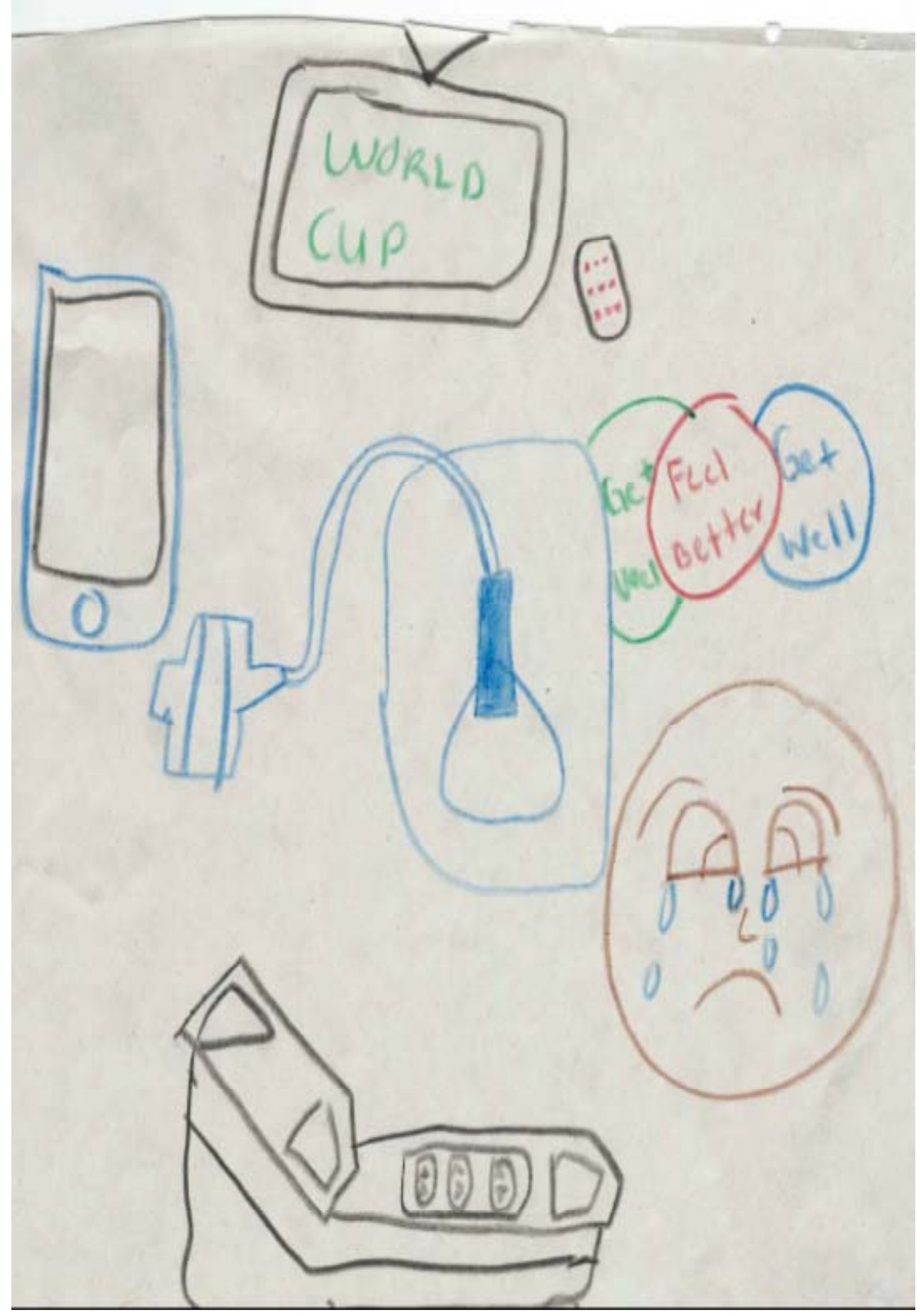


Appendix $\mathrm{H}$

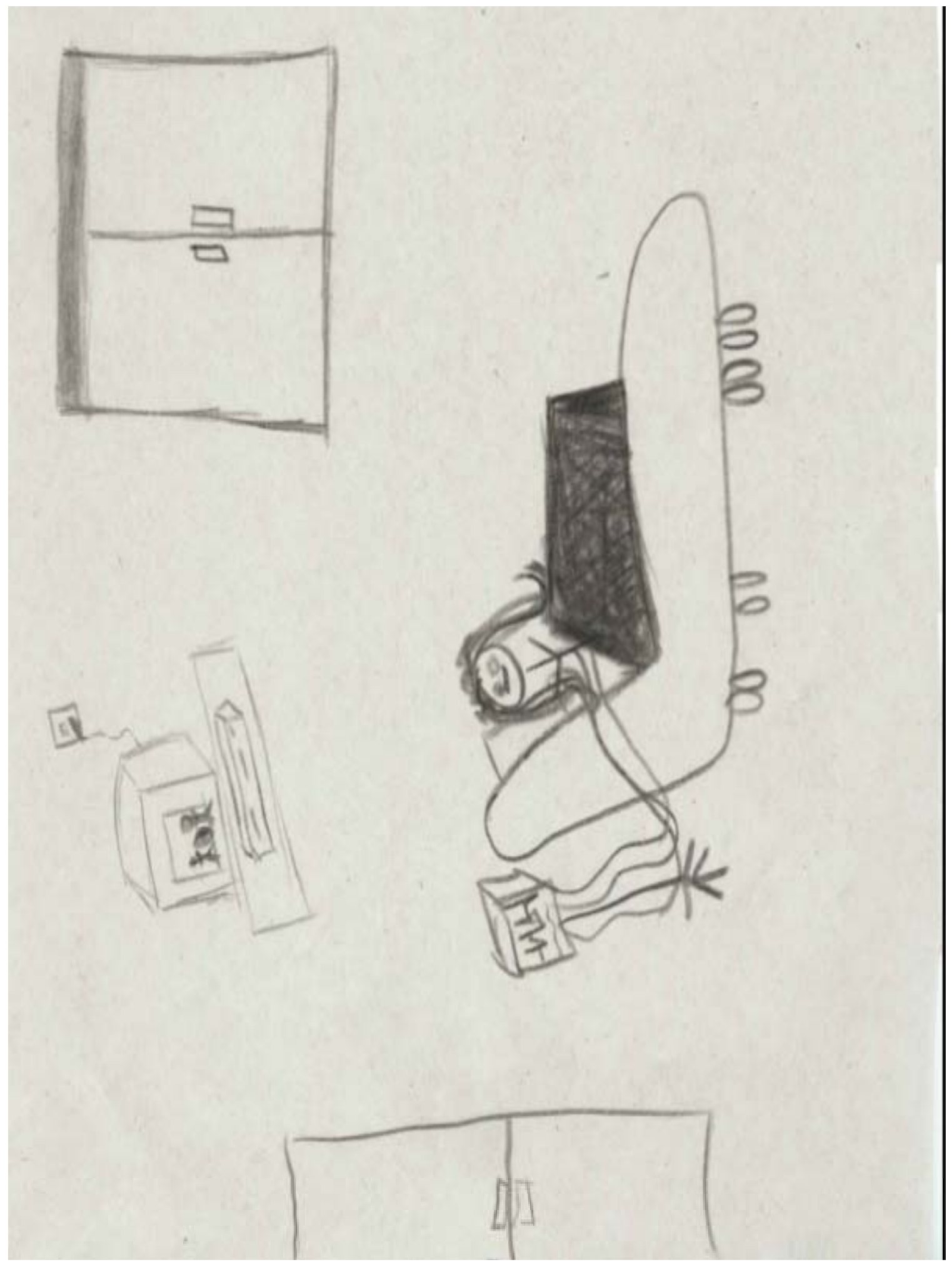


Appendix I

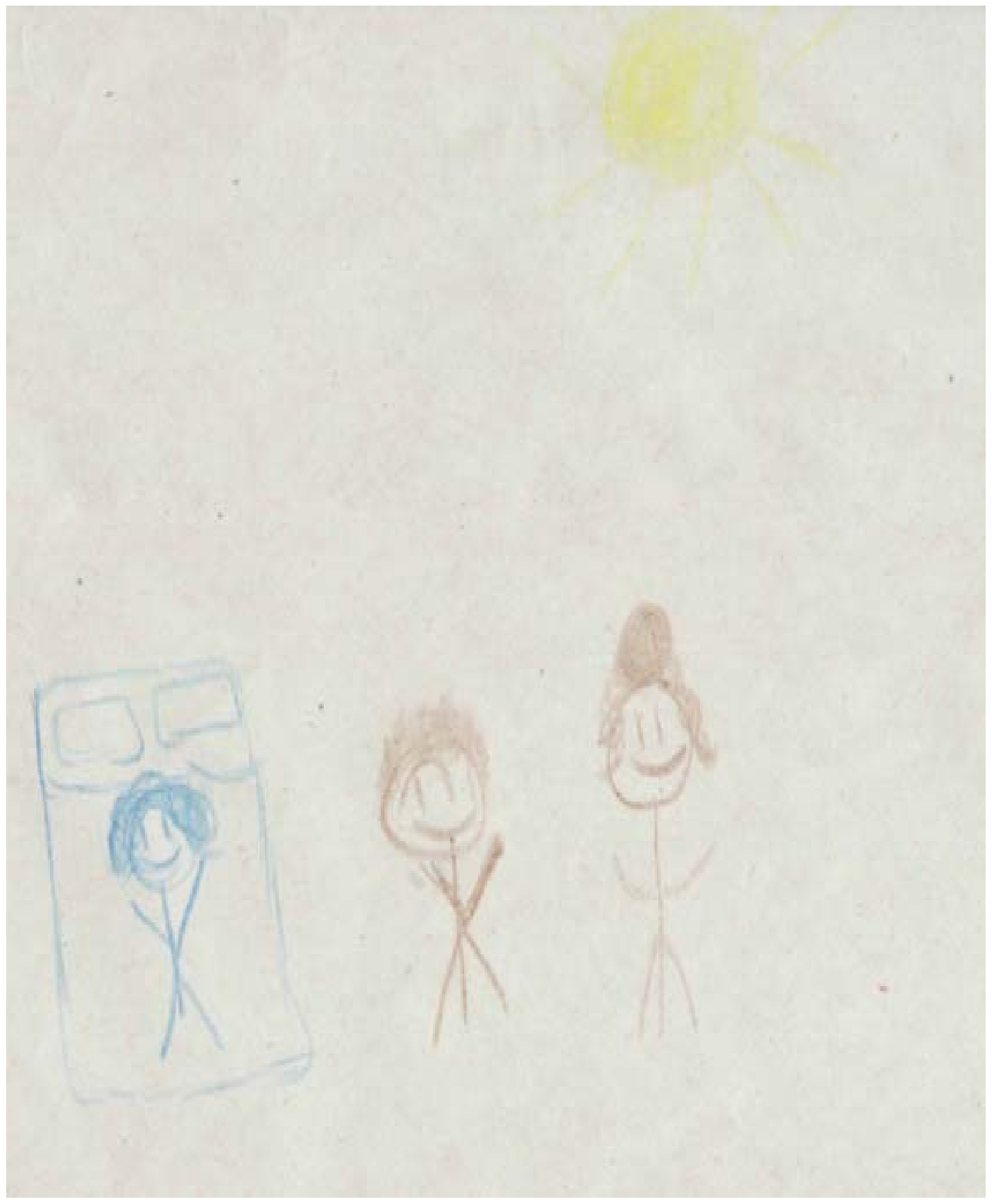


Appendix $\mathbf{J}$

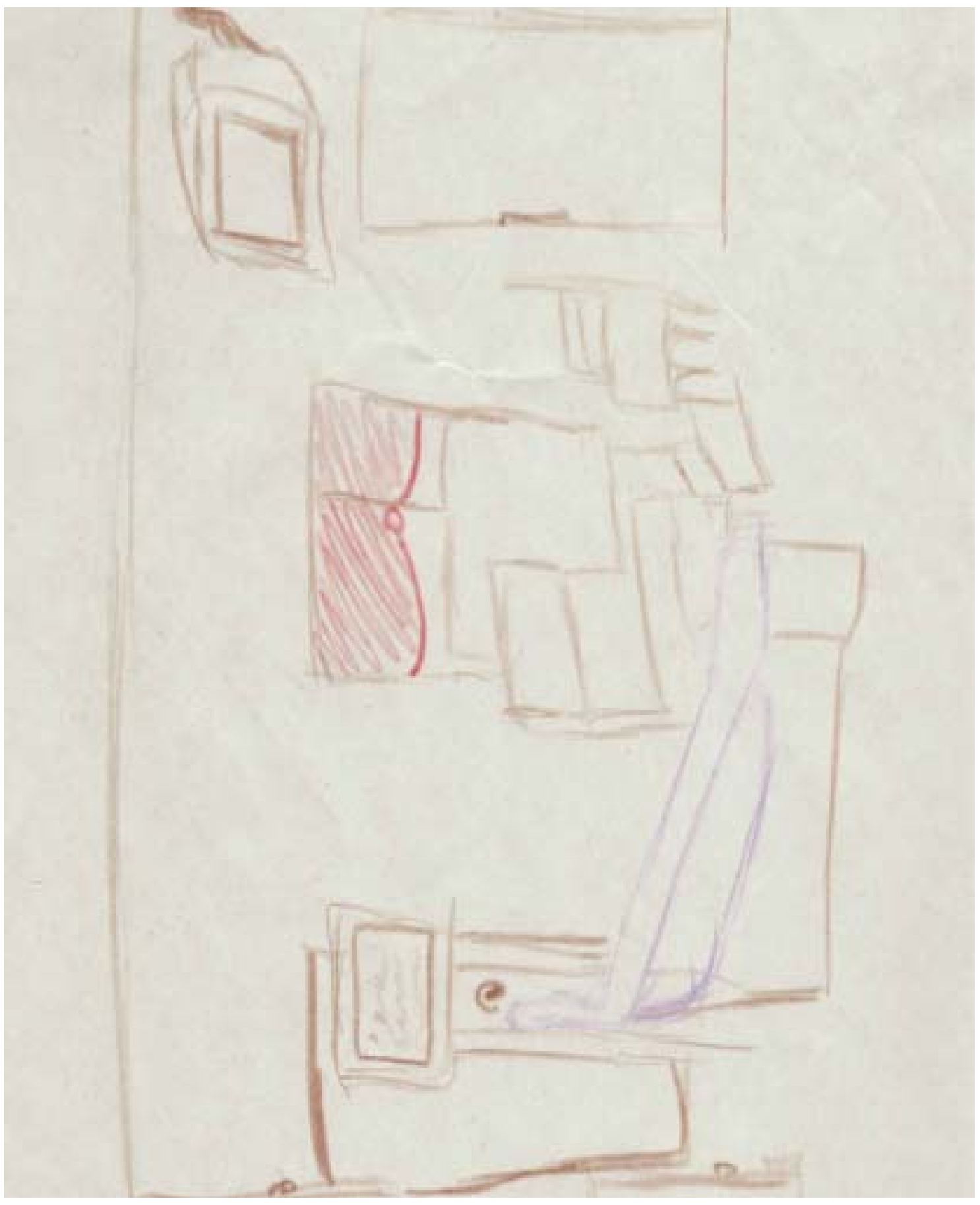


Appendix K

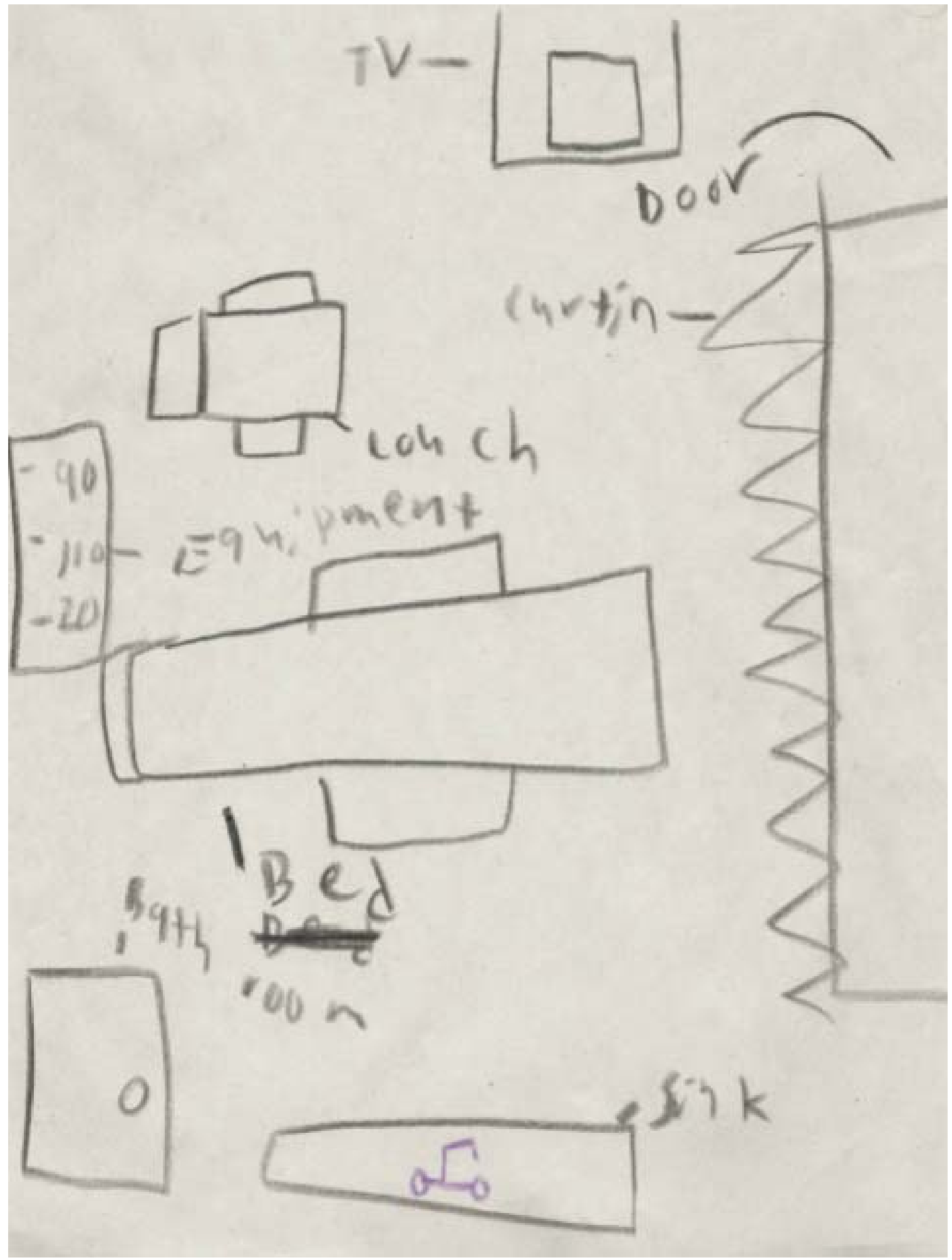


VITA

ANDREA S. PRENTISS

1976

Diploma in Nursing Jackson Memorial Hospital School of Nursing Miami, FL

1976-2002

Staff Nurse

Miami Children's Hospital

1993-2002

Staff Nurse

Baptist Health of Miami

2000-2002

Clinician/Educator

Homestead Hospital

2001

2002-2004

2004

2004-2006

Bachelors of Science in Nursing Florida International University

Miami, FL

Clinician/Care Coordinator

Baptist Health of Miami

Masters of Science in Nursing (MSN)

University of South Alabama

Mobile, AL

Advanced Patient Care Facilitator

Baptist Health of Miami

2006

Post Master's Certificate in Pediatric ARNP

Florida International University

Miami, FL

2006-2008

Nurse Specialist

Baptist Health of Miami

2008-2011

Clinical Nurse Specialist

2011 to present

Research Scientist

Baptist Health of South Florida 
PhD Candidate in Nursing

Florida International University

Miami, FL

\section{PUBLICATIONS AND PRESENTATIONS}

Prentiss, A. (2012). Early recognition of pediatric venous thromboembolism: A riskassessment tool. American Journal of Critical Care, 21(3), 178 - 185.

doi: $10.4037 /$ ajcc2012548

Seidu, M., Robinson-Trainor, L., \& Prentiss, A. Post-partum depression: Assessing for r: Poster. Presented at the Nursing Consortium of South Florida, Inc.; poster. 2/28/2014. Miami, FL.

Prentiss, A. Pediatric Head Trauma: Parent Reactions, Social Support, and Mental Health at Hospital Admission and Discharge. Presented at the SNRS 7th annual conference: poster. February 27, 2013. Little Rock, AR.

Caicedo, C., Youngblut, J.M., Brooten, D., Prentiss, A., \& Sanchez, N. (2012, September). Preschool Children Hospitalized with Head Injury: Functional Status and Type of Injury and Injury Severity. "Global Nursing Challenges for the Millennium," XIII Pan American Nursing Research Colloquium, international conference, Miami Beach, FL.

Prentiss, A., Butler, E., \& Kirgan, M. What are the Characteristics of an Extraordinary Nurse as Defined by Their Peers? Presented at the 30th anniversary of nursing at FIU Research Symposium: Poster. October 12, 2012. Miami, FL

Prentiss, A. Pediatric Venous Thromboembolism Risk Assessment Tool. Presented at the 2010 N.A.C.H.R.I. conference: Poster. Orlando, FL.

Prentiss, A. Refusal of Care Algorithm: Communication is key to safety. Presented at the 2007 N.A.C.H.R.I. conference: Poster. Miami, FL.

Prentiss, A. \& Butler, E. Crashing Kids: A Journey to a Successful Mock Code Program. Presented at the 2006 N.A.C.H.R.I conference: Poster. Alexandria, VA. 